\title{
Squaramide-Catalyzed Asymmetric Mannich Reactions of Azlactones with Isatin-Derived Ketimines: Access to $\alpha, \beta$-Diamino Acid Derivatives Bearing Vicinal Quaternary Stereocenters
}

\author{
Zhanhuan Li, ${ }^{\dagger}{ }^{\S}$ Jingyi Peng, ${ }^{\dagger, \S}$ Chonglong He,${ }^{\dagger}$ Jianfeng Xu ${ }^{\dagger}, *$ and Hongjun Ren $^{\ddagger * *}$ \\ 'Department of Chemistry, Zhejiang Sci-Tech University, Hangzhou 310018, P. R. China \\ ${ }^{\ddagger}$ Advanced Research Institute and Department of Chemistry, Taizhou University, 1139 Shifu Avenue, \\ Taizhou 318000, P. R. China \\ *E-mail: jfxu@zstu.edu.cn. \\ *E-mail: renhj@zstu.edu.cn and renhj@tzc.edu.cn.
}

Table of Contents

\begin{tabular}{|c|cc|}
\hline I & X-ray structure of product 3I & S2 \\
\hline II & NMR spectra & S5 \\
\hline III & HPLC spectra & S34 \\
\hline
\end{tabular}




\section{X-ray structure of product 31 (ellipsoid contour at $30 \%$ probability)}

\section{Experimental}

Single crystals of $3 \mathbf{1}\left(\mathrm{C}_{28} \mathrm{H}_{32} \mathrm{FN}_{3} \mathrm{O}_{5}\right)$ were grown by slow evaporation in petroleum ether $/ \mathrm{CH}_{2} \mathrm{Cl}_{2}=2 / 1$ under air atmosphere. A suitable crystal was selected and mounted on a diffractometer. The crystal was kept at $293 \mathrm{~K}$ during data collection. Using SHELXL, the structure was solved with the XS structure solution program using Direct Methods and refined with the XL refinement package using Least Squares minimisation.

\section{Crystal structure determination}

Crystal Data for 3I (CCDC 1977810): $\mathrm{C}_{28} \mathrm{H}_{32} \mathrm{FN}_{3} \mathrm{O}_{5}, \quad M=509.56 \mathrm{~g} / \mathrm{mol}$, orthorhombic, space group P $212121, a=10.60302(19) \AA, b=10.85734(19) \AA, c=$ 24.9687(4) $\AA, \alpha=90^{\circ}, \beta=90^{\circ}, \gamma=90^{\circ}, V=2874.41(8) \AA^{3}, Z=4, T=293(2) \mathrm{K}$, $\mu(\mathrm{CuK} \alpha)=0.706 \mathrm{~mm}^{-1}, D=1.177 \mathrm{~g} / \mathrm{cm}^{3}$, reflections collected/unique $16781 / 5126$ $(R$ int $=0.0146)$, final $R$ indices $[\mathrm{I}>2 \sigma(\mathrm{I})], R_{1}=0.0390, w R_{2}=0.1089 ; R$ indices (all data), $R_{1}=0.0398, w R_{2}=0.1102$. 
Figure S1. ORTEP diagram of 31 with ellipsoid contour at $30 \%$ probability level (CCDC 1977810)
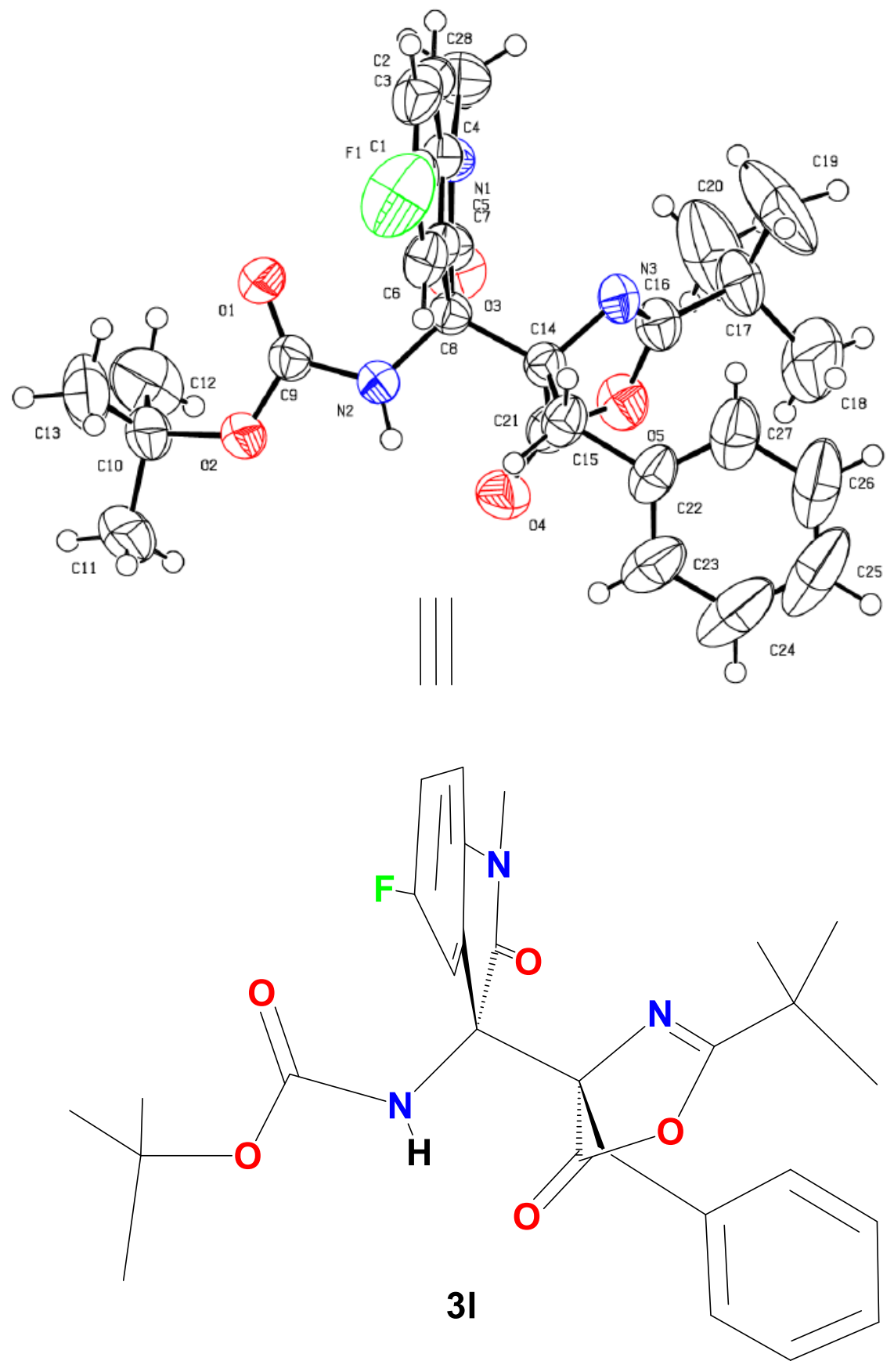
Table S1. Crystal data and structure refinement for 31 .

Empirical formula

Formula weight

Temperature

Wavelength

Crystal system, Space group

Unit cell dimensions

Volume

Z

Density(calculated)

Absorption coefficient

$\mathrm{F}(000)$

Crystal size

Theta range for data collection

Index ranges

Reflections collected

Independent reflections

Completeness to theta $=67.23^{\circ}$

Absorption correction

Max. and min. transmission

Refinement method

Data/restraints/parameters

Goodness-of-fit on F2

Final R indices [I $>2 \operatorname{sigma}(\mathrm{I})]$

$\mathrm{R}$ indices (all data)

Extinction coefficient

Largest diff. peak and hole
$\mathrm{C}_{28} \mathrm{H}_{32} \mathrm{FN}_{3} \mathrm{O}_{5}$

509.56

293(2) K

1.54184

orthorhombic,

P 212121

$\mathrm{a}=10.60302(19) \quad \alpha=90^{\circ}$

$\mathrm{b}=10.85734(19) \quad \beta=90^{\circ}$

$\mathrm{c}=24.9687(4) \quad \gamma=90^{\circ}$

2874.41(8)

4

$1.177 \mathrm{Mg} / \mathrm{m}^{3}$

$0.706 \mathrm{~mm}^{-1}$

1080.0

$0.12 * 0.11 * 0.11 \mathrm{~mm}^{3}$

4.53 to $67.23^{\circ}$.

$-12<=\mathrm{h}<=8,-12<=\mathrm{k}<=12,-29<=\mathrm{l}<=29$

16781

$5126[R($ int $)=0.0146]$

$99.7 \%$

Multi-scan from equivalents

0.926 and 0.920

Full-matrix least-squares on $\mathrm{F}^{\wedge} 2$

$5126 / 0 / 341$

1.022

$R l=0.0390, w R 2=0.1089$

$R l=0.0398, w R 2=0.1102$

$\mathrm{n} / \mathrm{a}$

0.225 and -0.183 e. $\AA^{-3}$ 


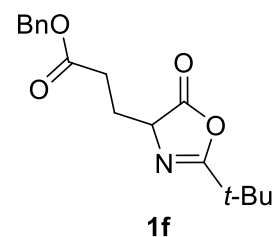

$\mathrm{CDCl}_{3},{ }^{1} \mathrm{H} \mathrm{NMR}, 400 \mathrm{MHz}$

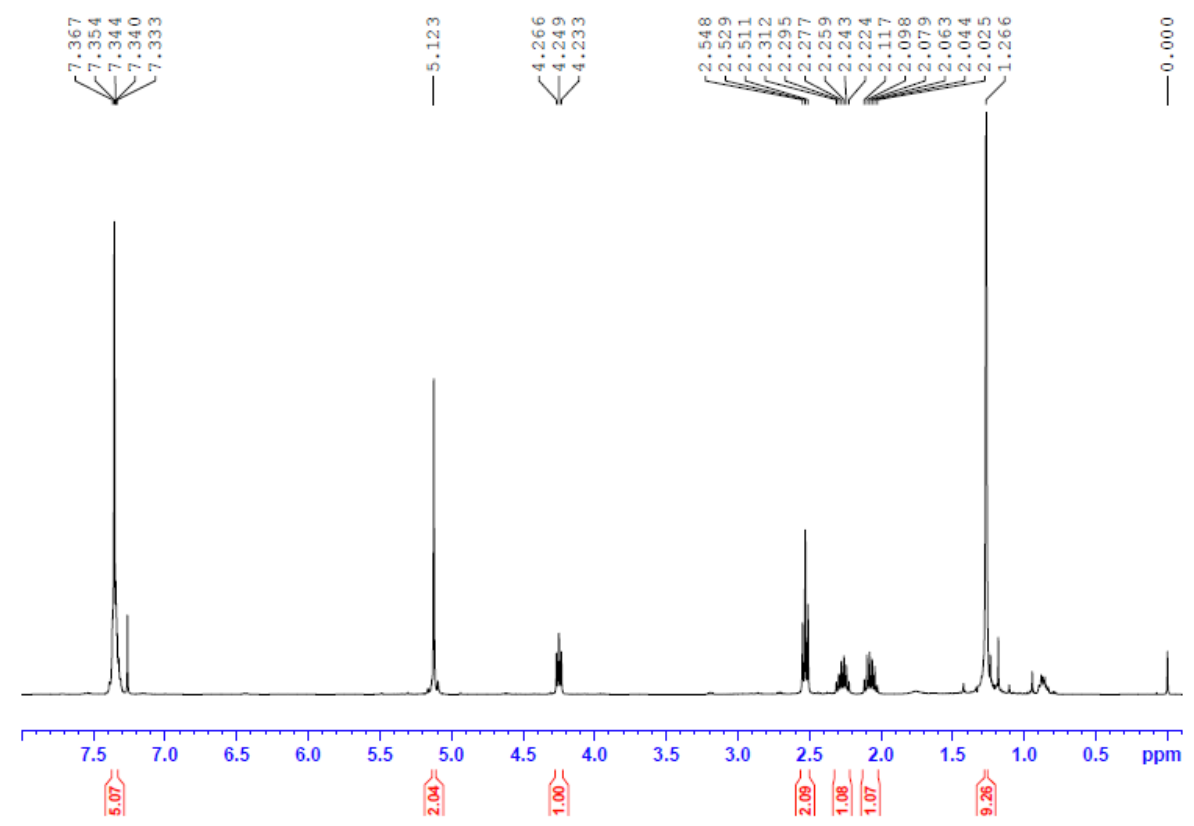

$\mathrm{CDCl}_{3},{ }^{13} \mathrm{C}\{1 \mathrm{H}\} \mathrm{NMR}, 100 \mathrm{MHz}$

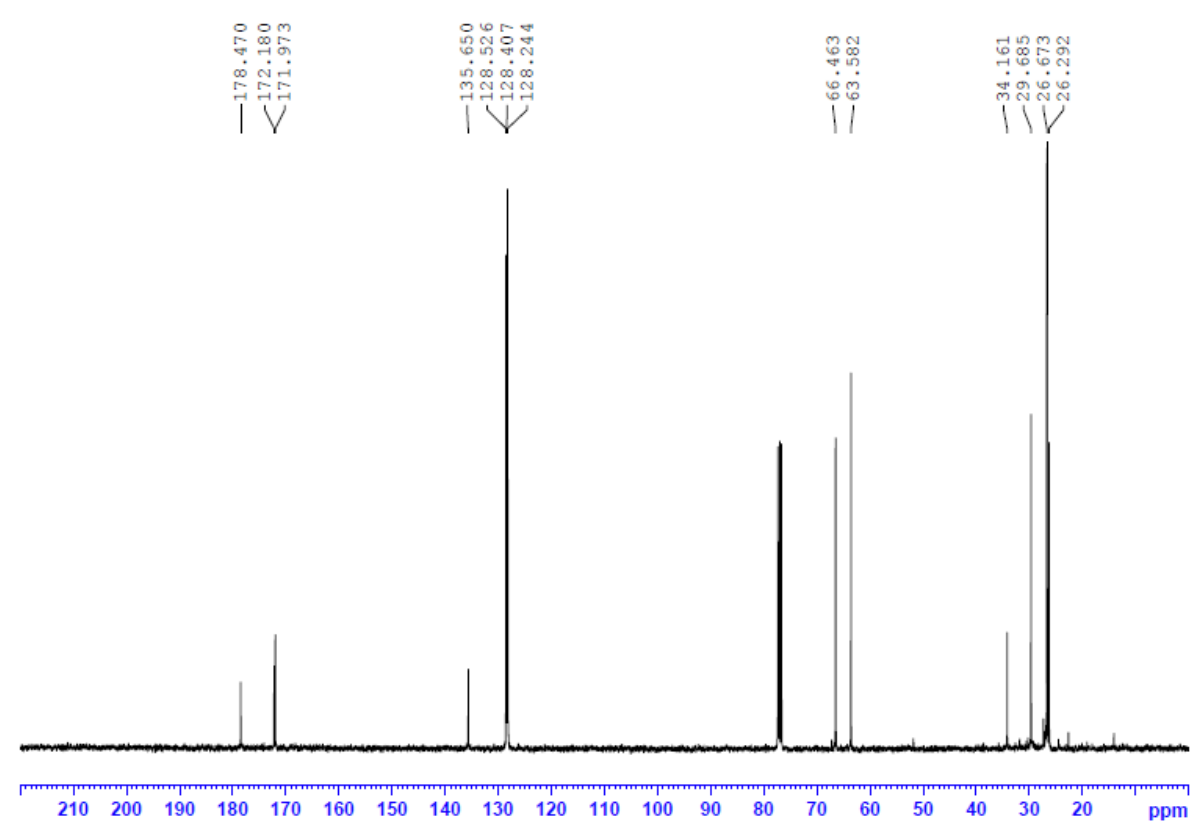




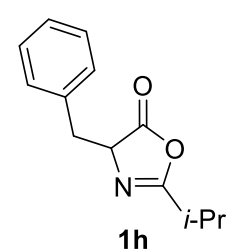

$\mathrm{CDCl}_{3},{ }^{1} \mathrm{H}$ NMR, $400 \mathrm{MHz}$

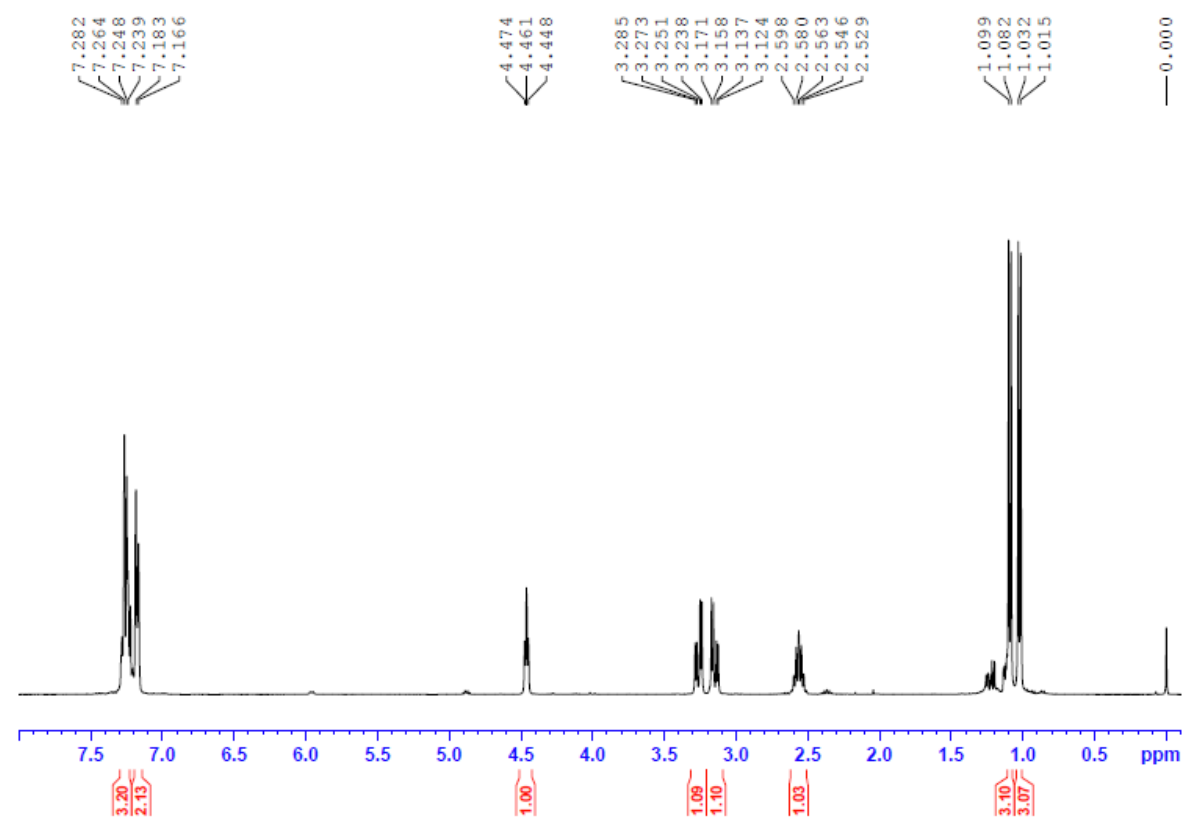

$\mathrm{CDCl}_{3},{ }^{13} \mathrm{C}\{1 \mathrm{H}\}$ NMR, $100 \mathrm{MHz}$
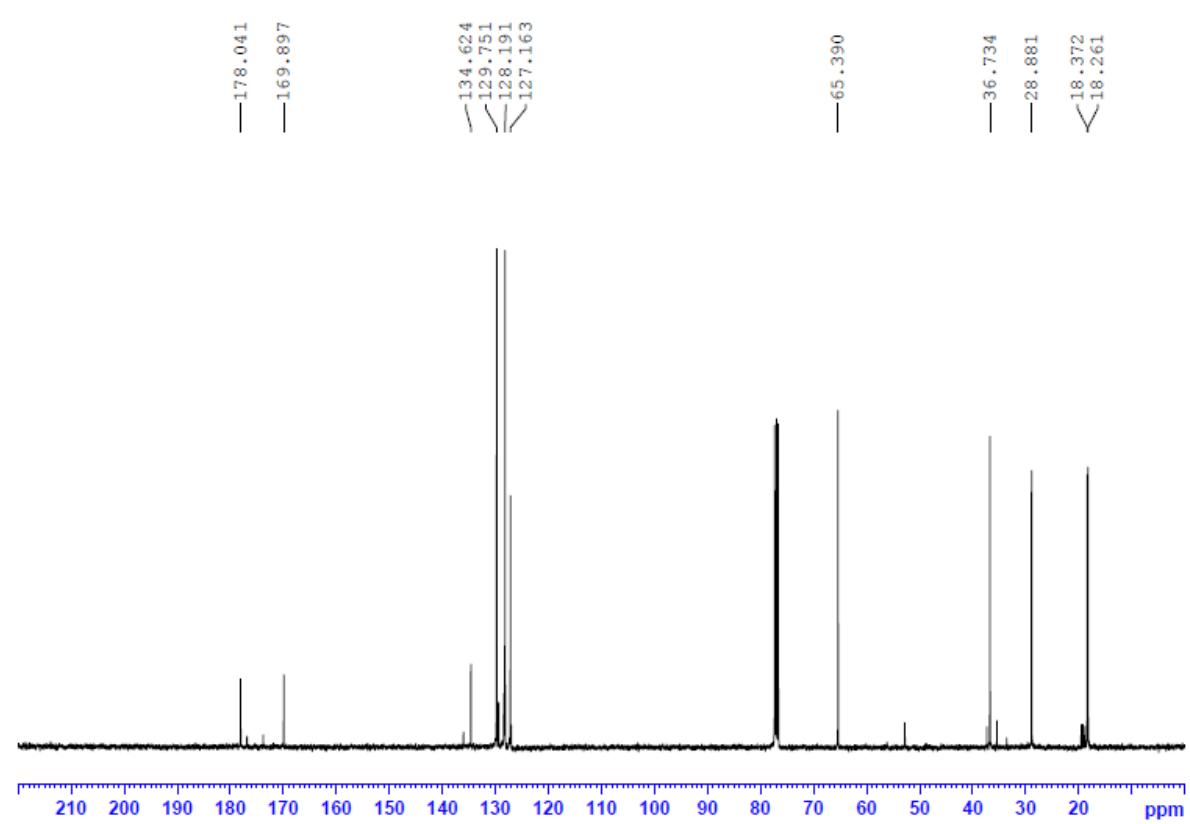


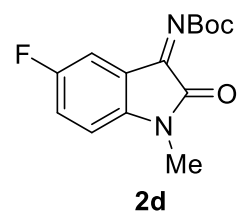

$\mathrm{CDCl}_{3},{ }^{1} \mathrm{H}$ NMR, $400 \mathrm{MHz}$

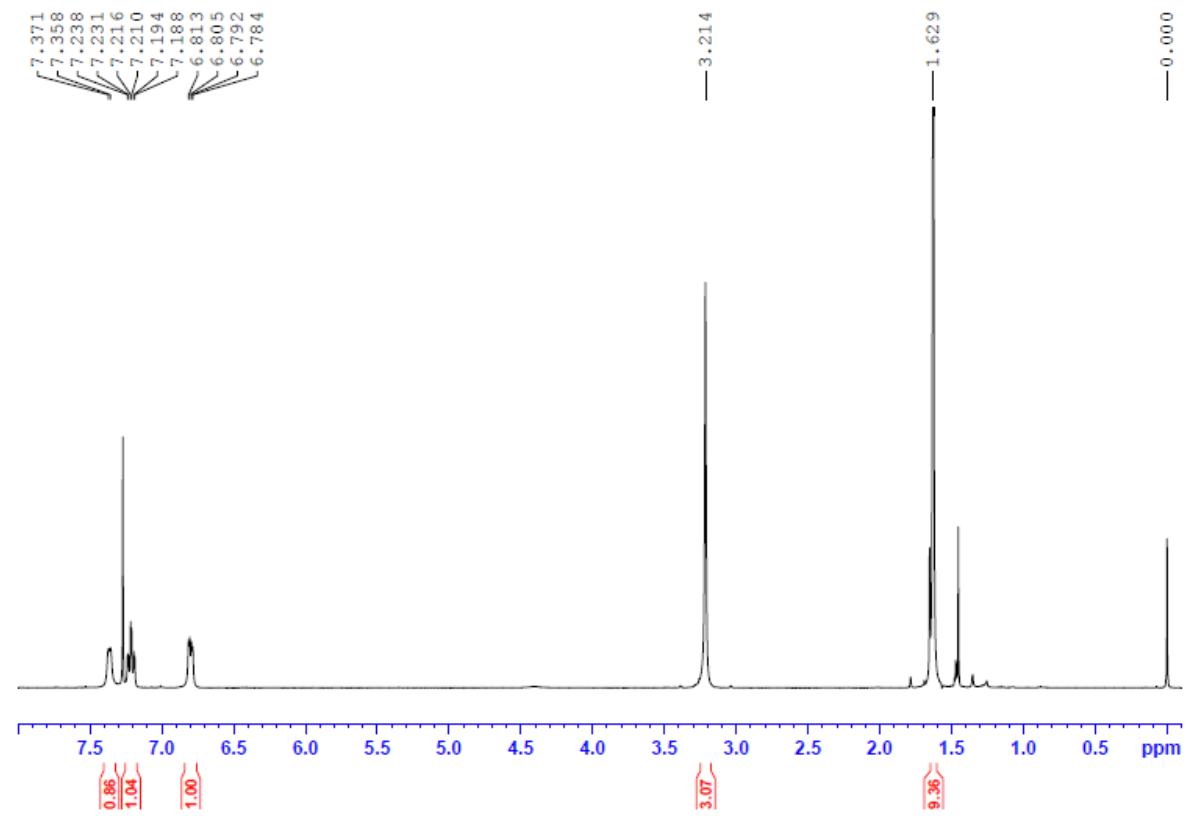

$\mathrm{CDCl}_{3},{ }^{13} \mathrm{C}\{1 \mathrm{H}\}$ NMR, $100 \mathrm{MHz}$

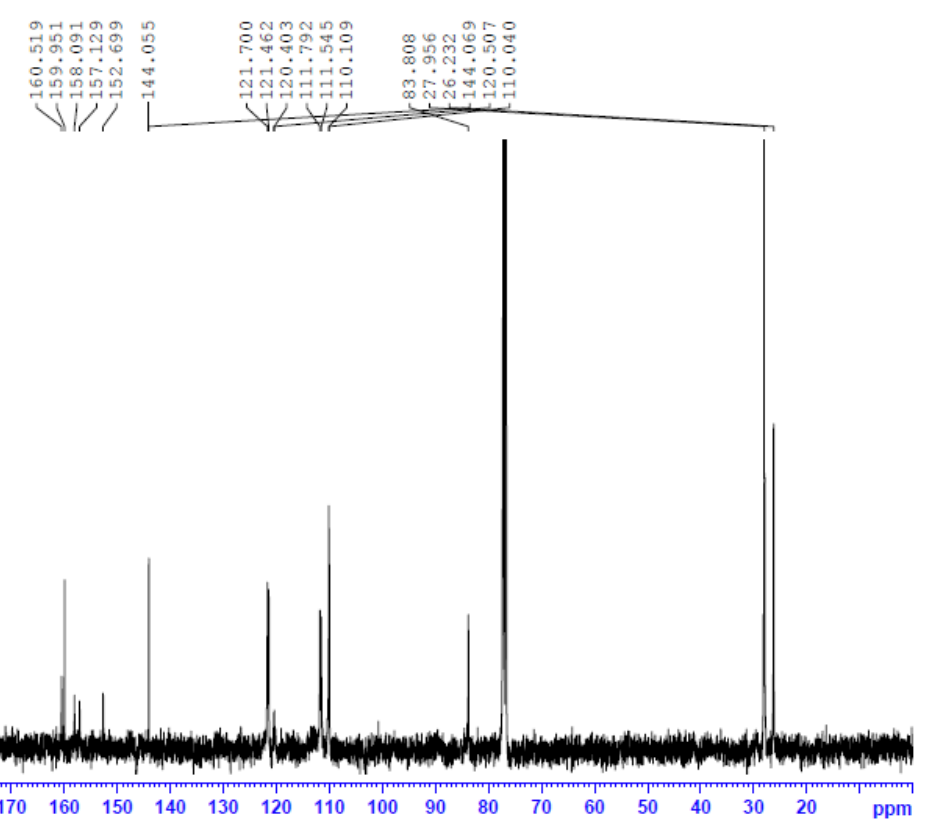




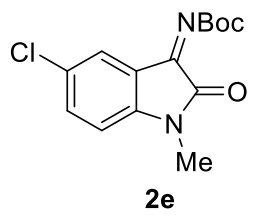

$\mathrm{CDCl}_{3},{ }^{1} \mathrm{H}$ NMR, $400 \mathrm{MHz}$

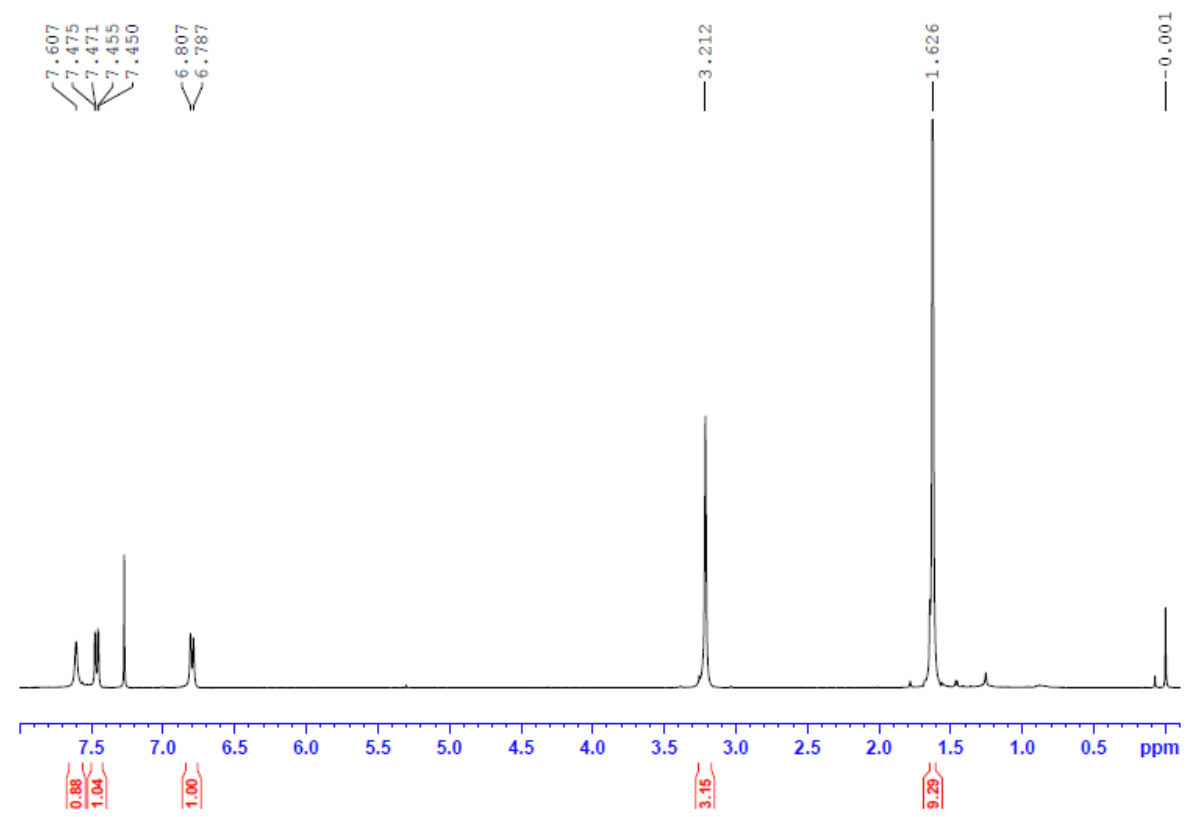

$\mathrm{CDCl}_{3},{ }^{13} \mathrm{C}\{1 \mathrm{H}\} \mathrm{NMR}, 100 \mathrm{MHz}$

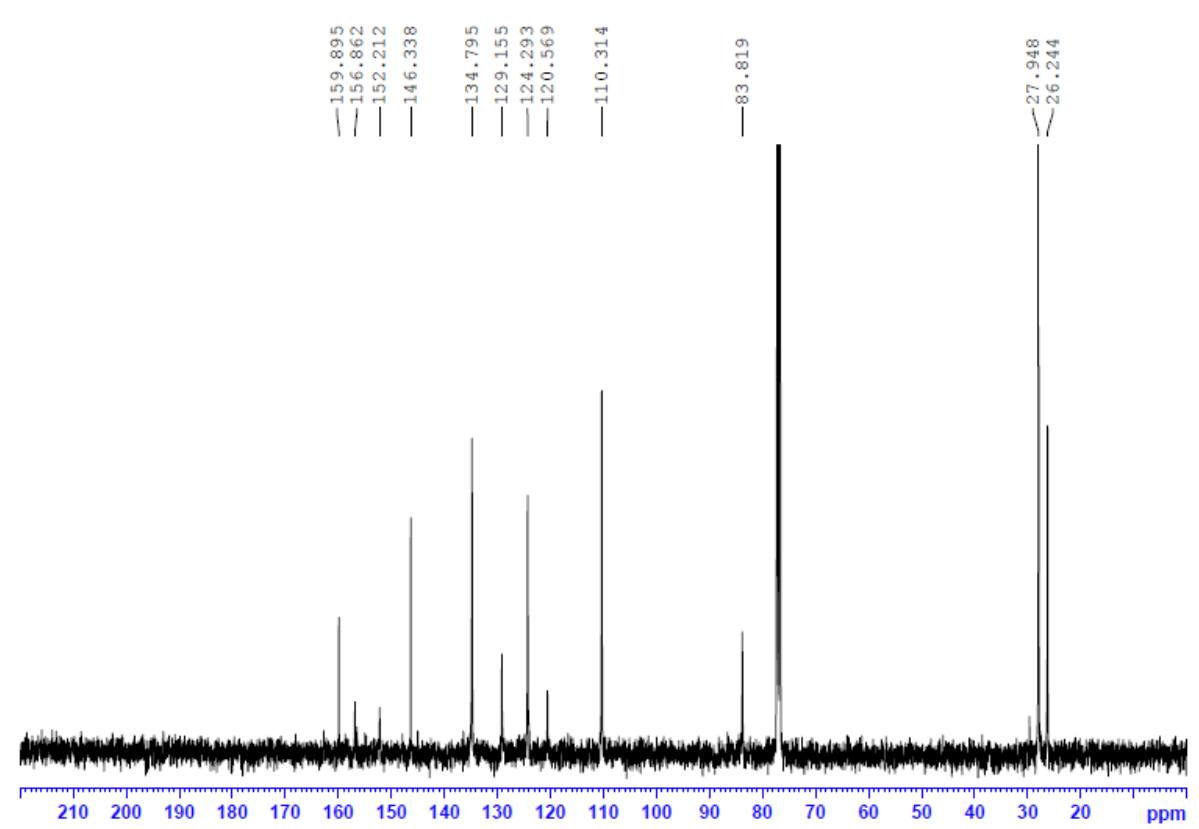




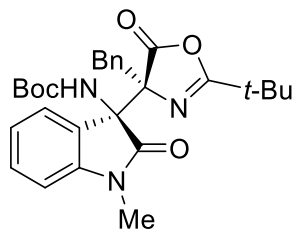

3a

$\mathrm{CDCl}_{3},{ }^{1} \mathrm{H} \mathrm{NMR}, 400 \mathrm{MHz}$

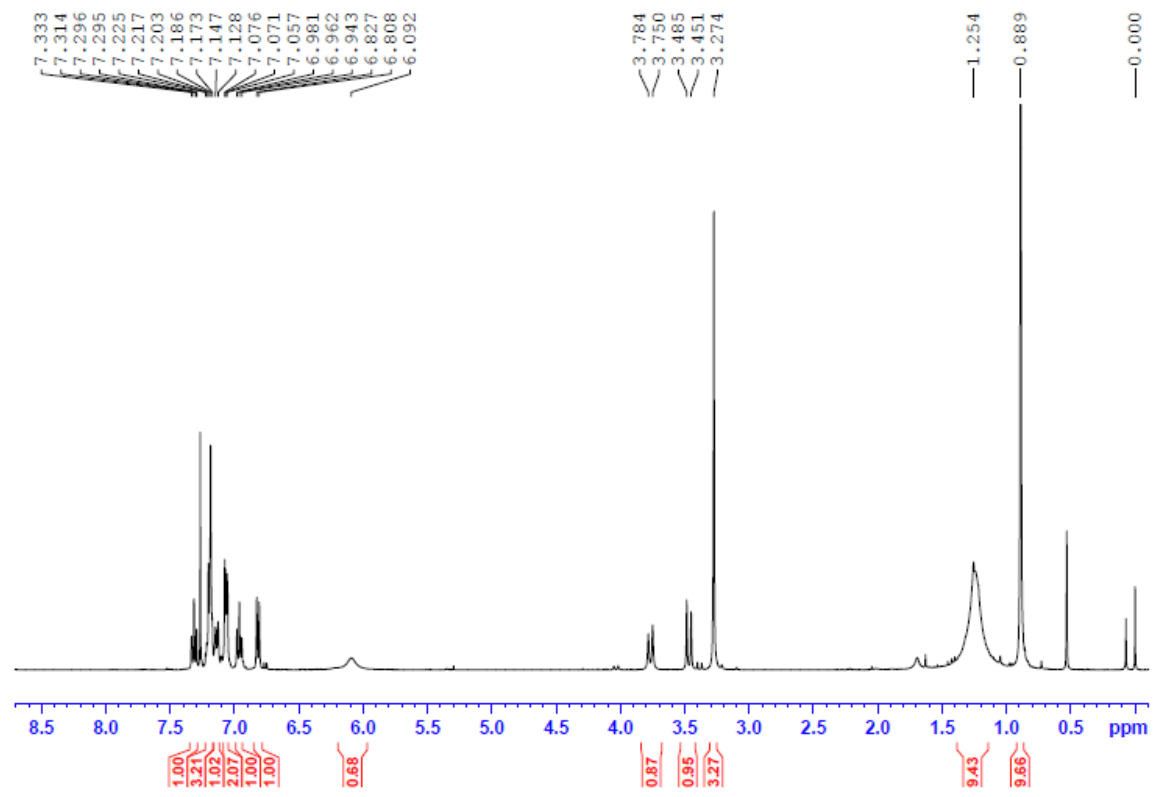

$\mathrm{CDCl}_{3},{ }^{13} \mathrm{C}\{1 \mathrm{H}\} \mathrm{NMR}, 100 \mathrm{MHz}$

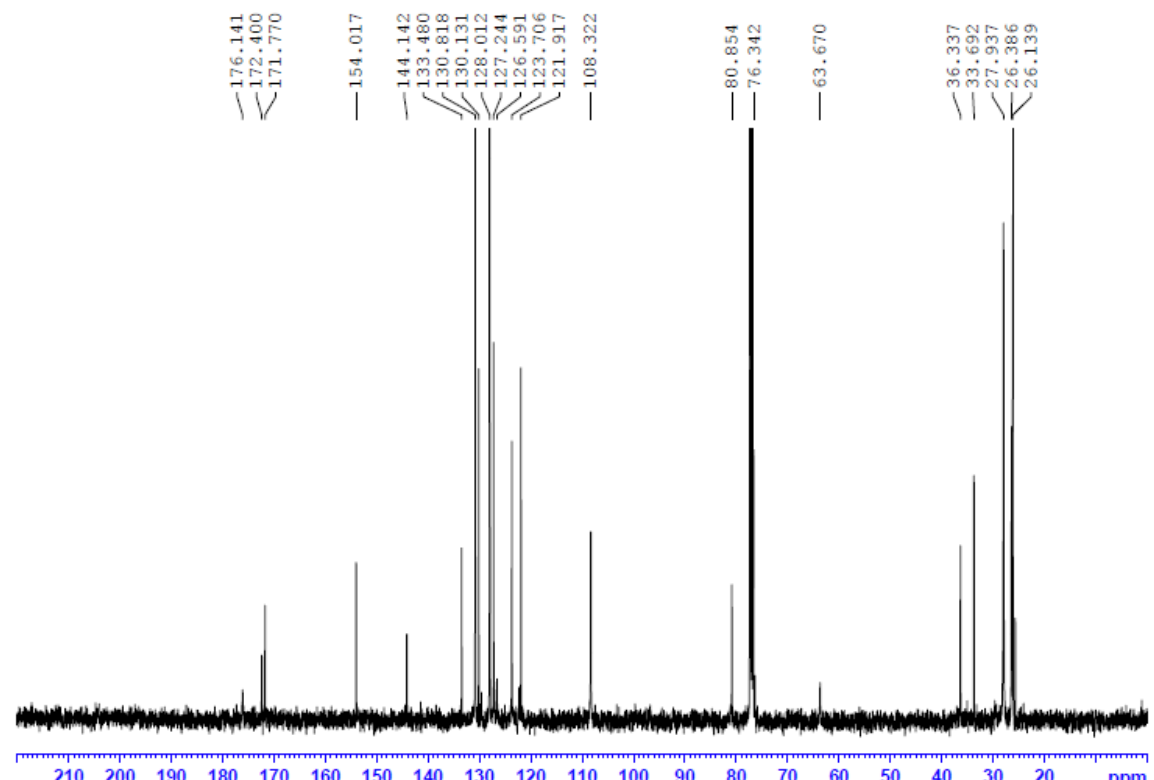

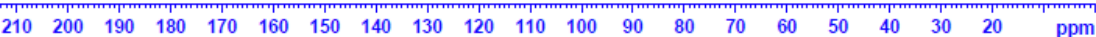




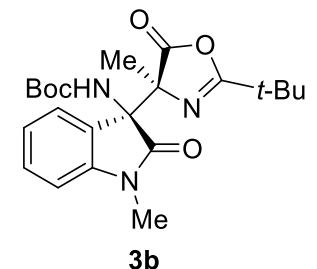

$\mathrm{CDCl}_{3},{ }^{1} \mathrm{H} \mathrm{NMR}, 400 \mathrm{MHz}$

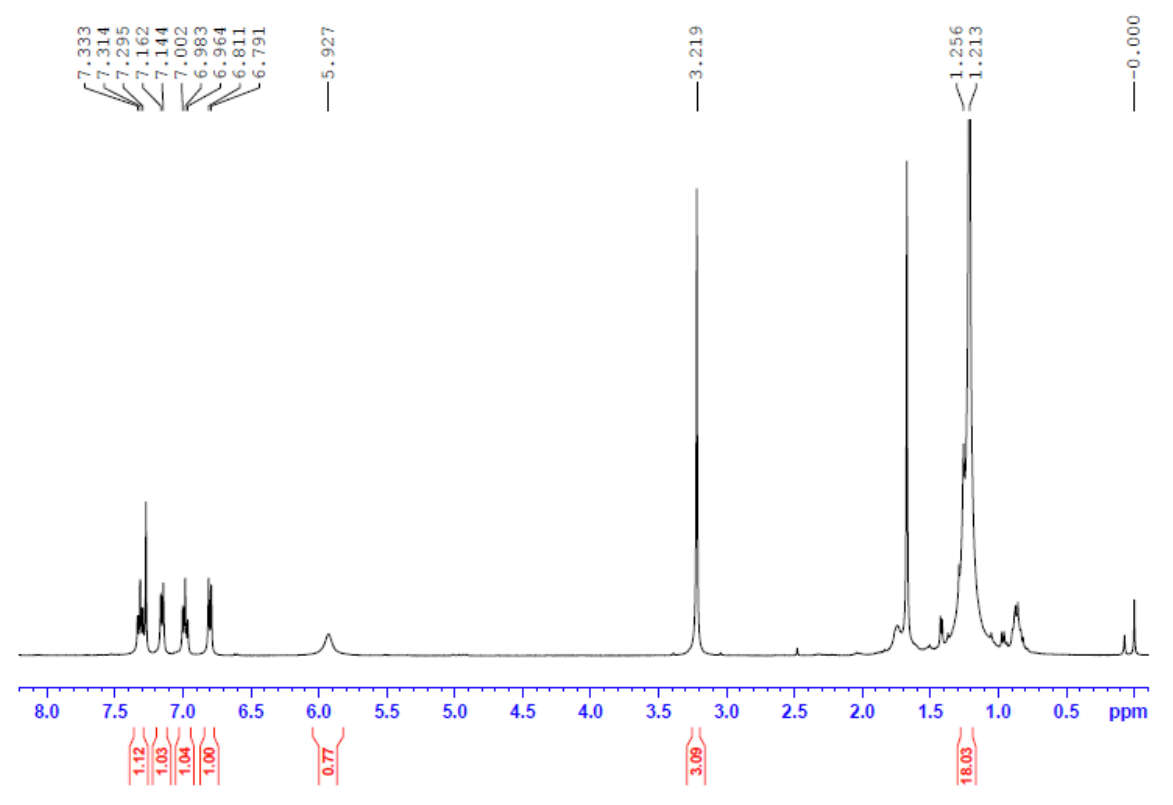

$\mathrm{CDCl}_{3},{ }^{13} \mathrm{C}\{1 \mathrm{H}\} \mathrm{NMR}, 100 \mathrm{MHz}$

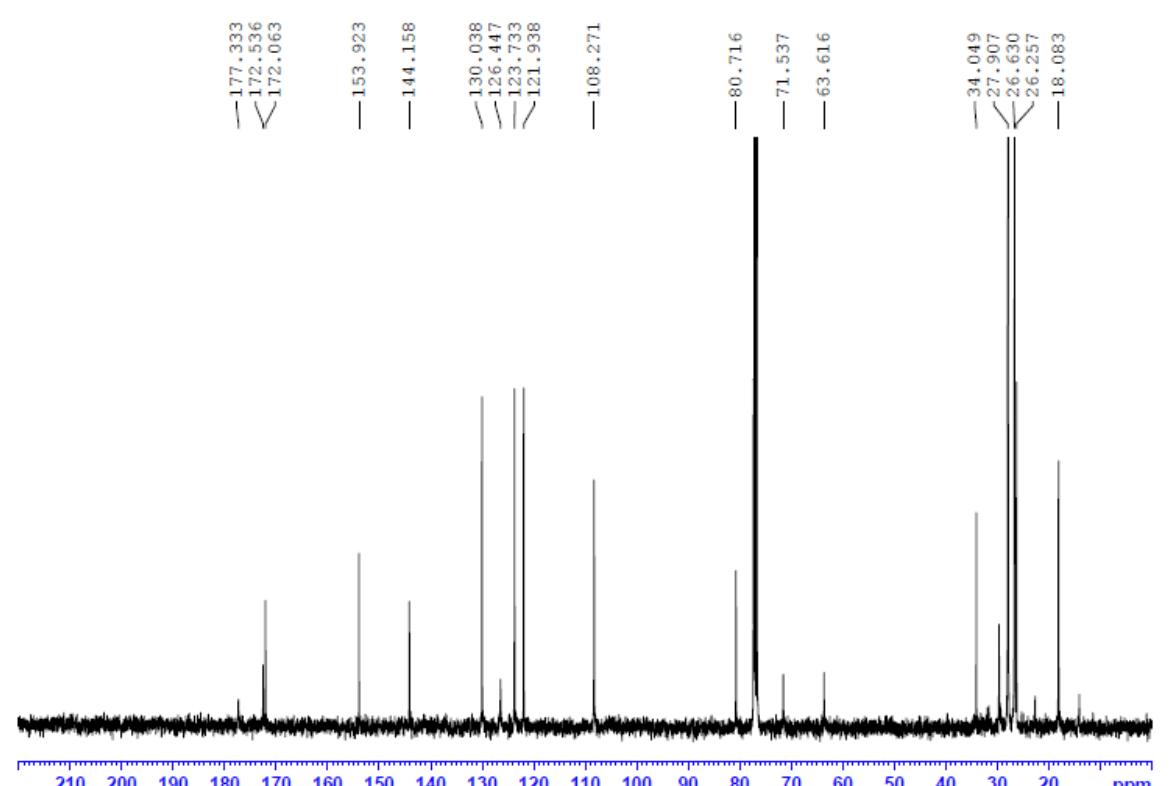

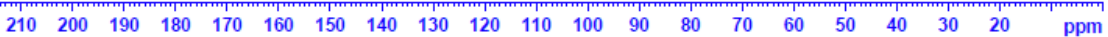




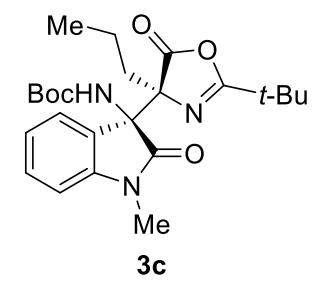

$\mathrm{CDCl}_{3},{ }^{1} \mathrm{H} \mathrm{NMR}, 400 \mathrm{MHz}$

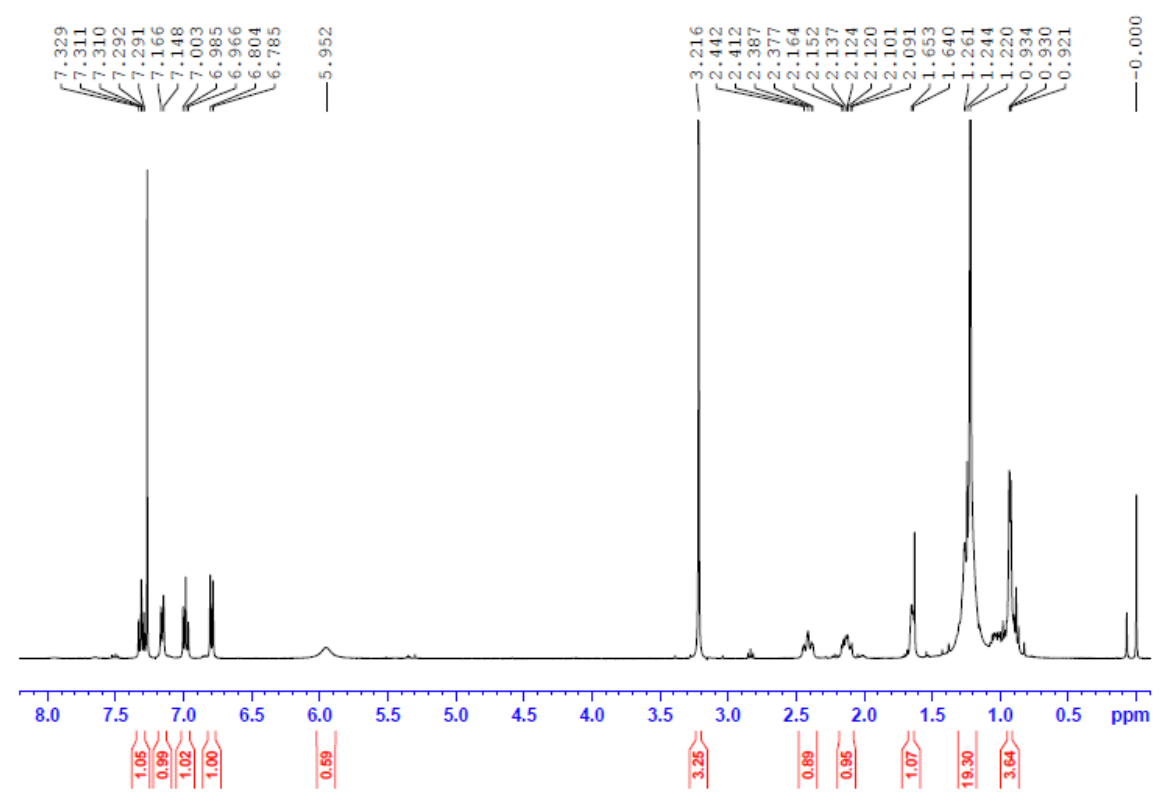

$\mathrm{CDCl}_{3},{ }^{13} \mathrm{C}\{1 \mathrm{H}\} \mathrm{NMR}, 100 \mathrm{MHz}$

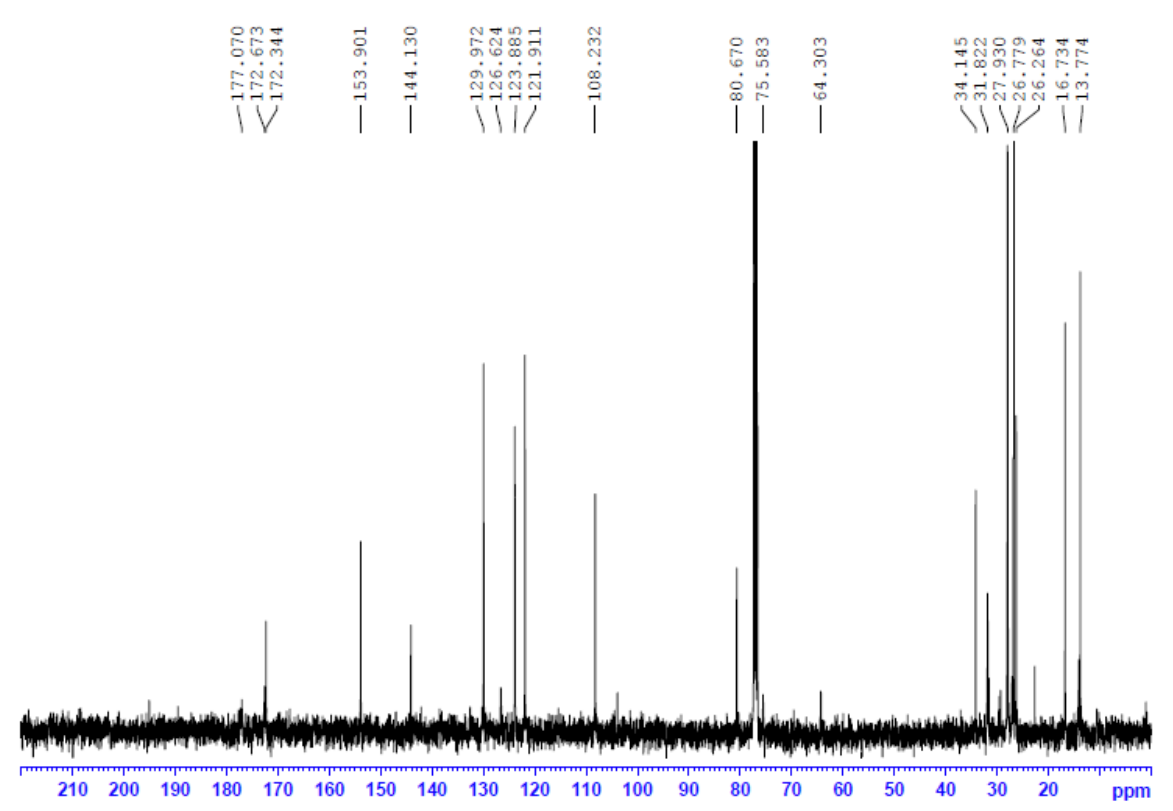




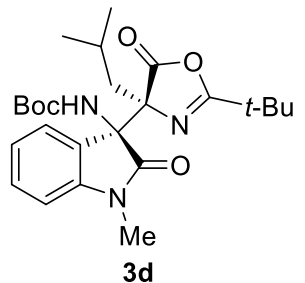

$\mathrm{CDCl}_{3},{ }^{1} \mathrm{H} \mathrm{NMR}, 400 \mathrm{MHz}$

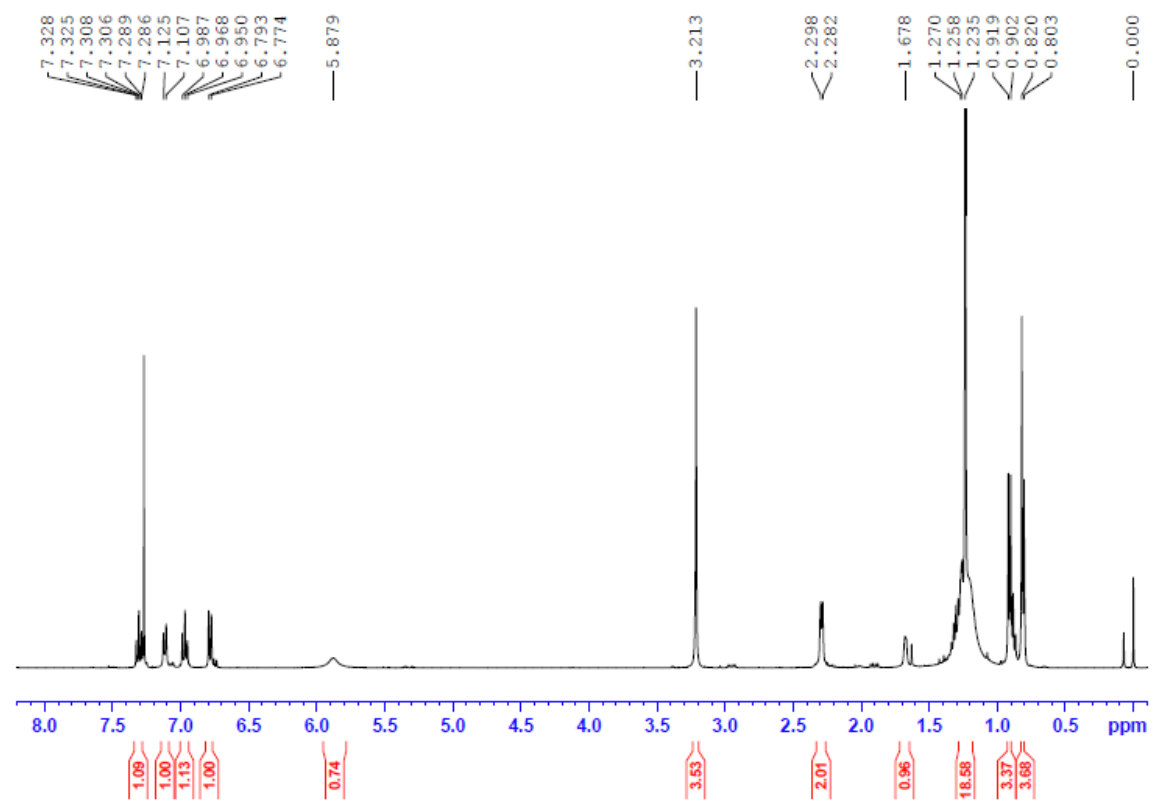

$\mathrm{CDCl}_{3},{ }^{13} \mathrm{C}\{1 \mathrm{H}\} \mathrm{NMR}, 100 \mathrm{MHz}$

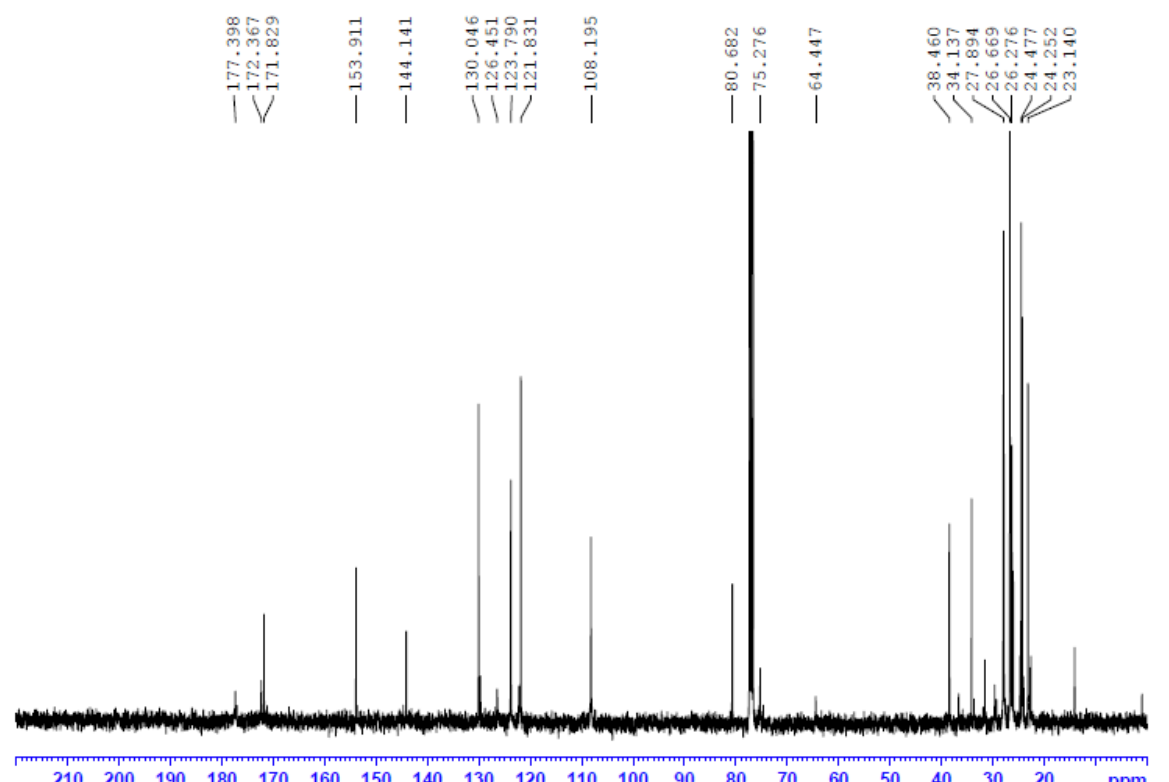




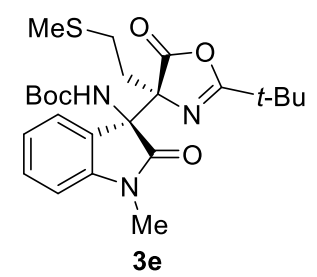

$\mathrm{CDCl}_{3},{ }^{1} \mathrm{H} \mathrm{NMR}, 400 \mathrm{MHz}$

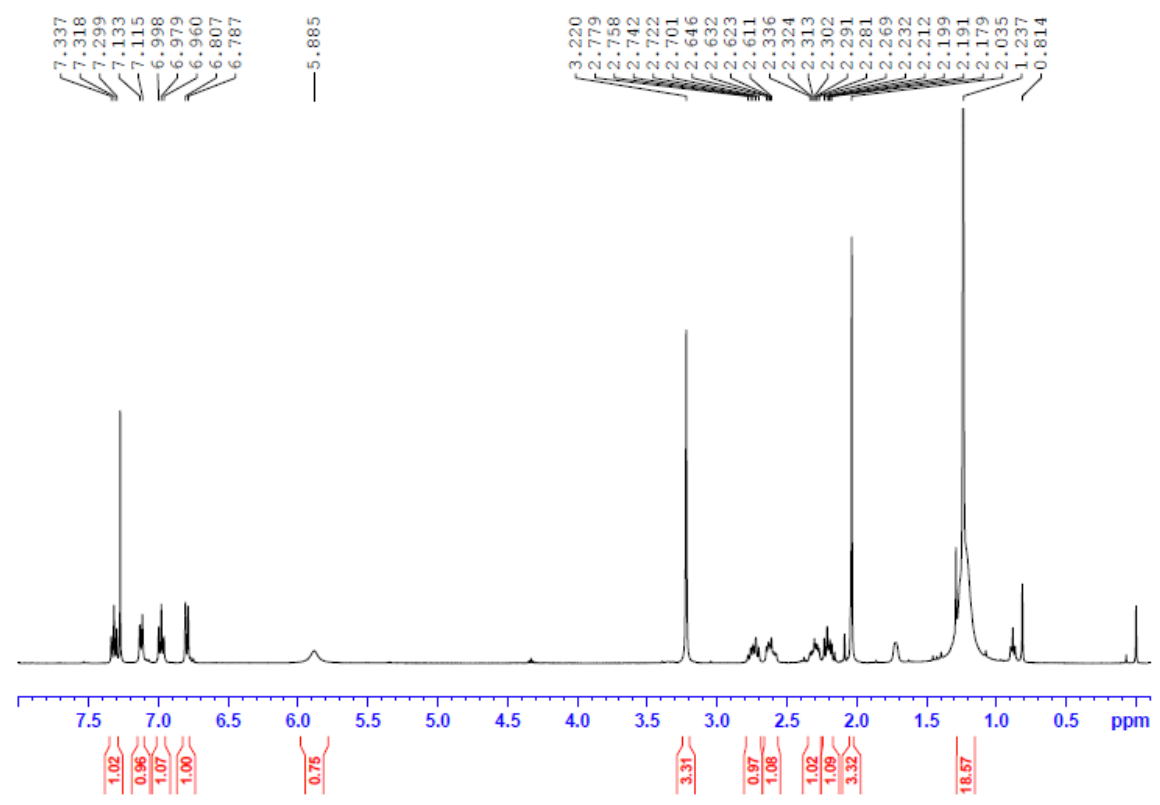

$\mathrm{CDCl}_{3},{ }^{13} \mathrm{C}\{1 \mathrm{H}\} \mathrm{NMR}, 100 \mathrm{MHz}$

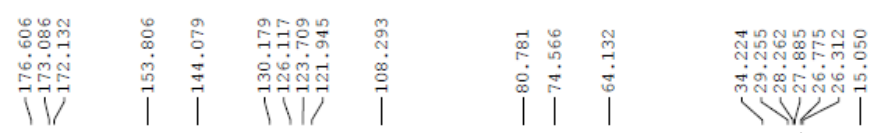

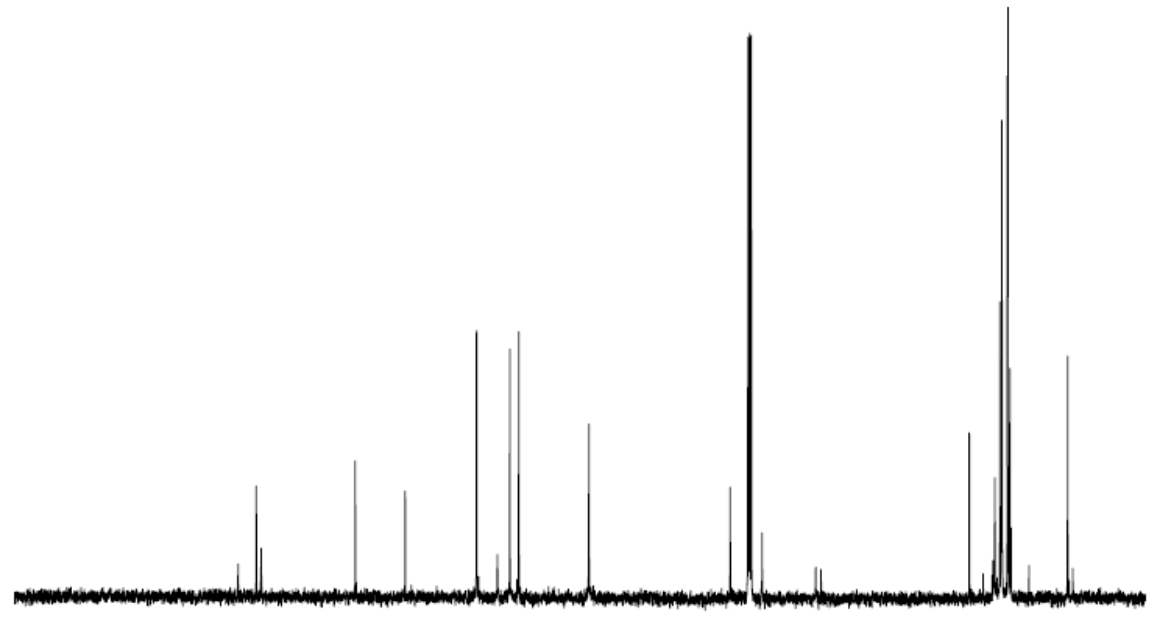

$\begin{array}{lllllllllllllllllllll}210 & 200 & 190 & 180 & 170 & 160 & 150 & 140 & 130 & 120 & 110 & 100 & 90 & 80 & 70 & 60 & 50 & 40 & 30 & 20 & \text { ppm }\end{array}$ 


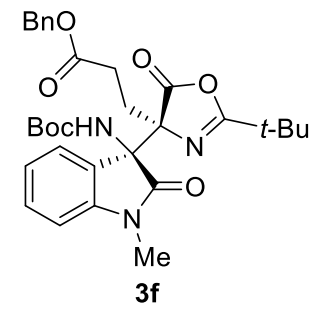

$\mathrm{CDCl}_{3},{ }^{1} \mathrm{H} \mathrm{NMR}, 400 \mathrm{MHz}$

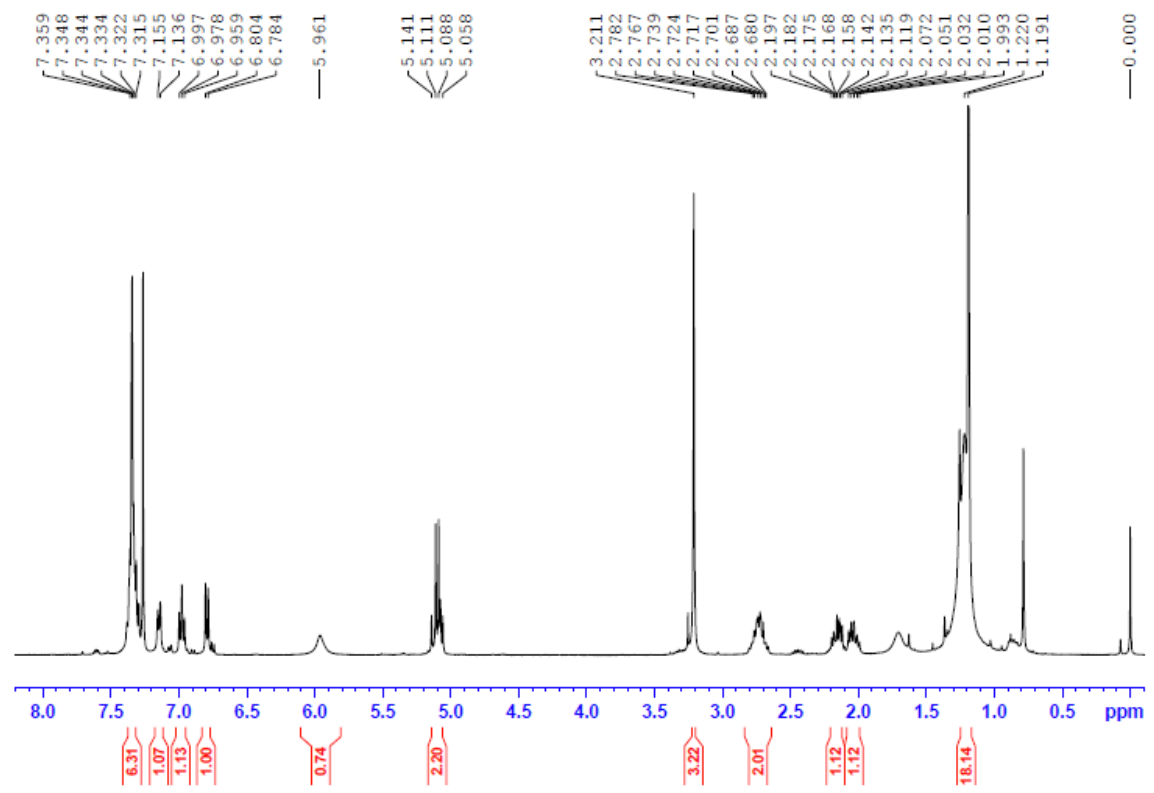

$\mathrm{CDCl}_{3},{ }^{13} \mathrm{C}\{1 \mathrm{H}\} \mathrm{NMR}, 100 \mathrm{MHz}$

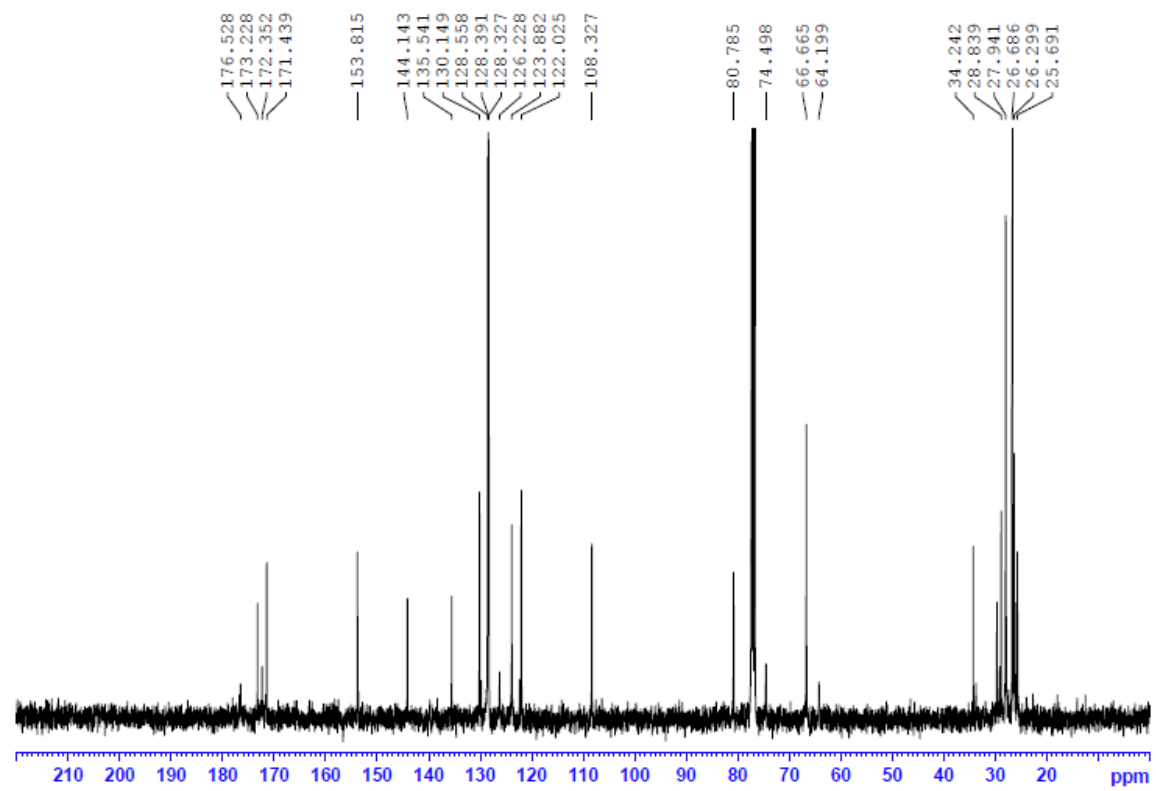




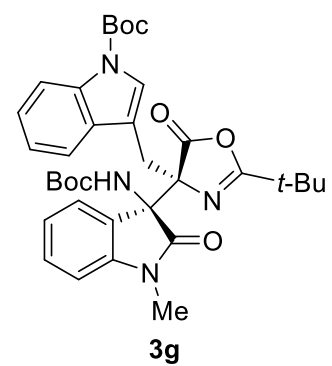

$\mathrm{CDCl}_{3},{ }^{1} \mathrm{H} \mathrm{NMR}, 400 \mathrm{MHz}$

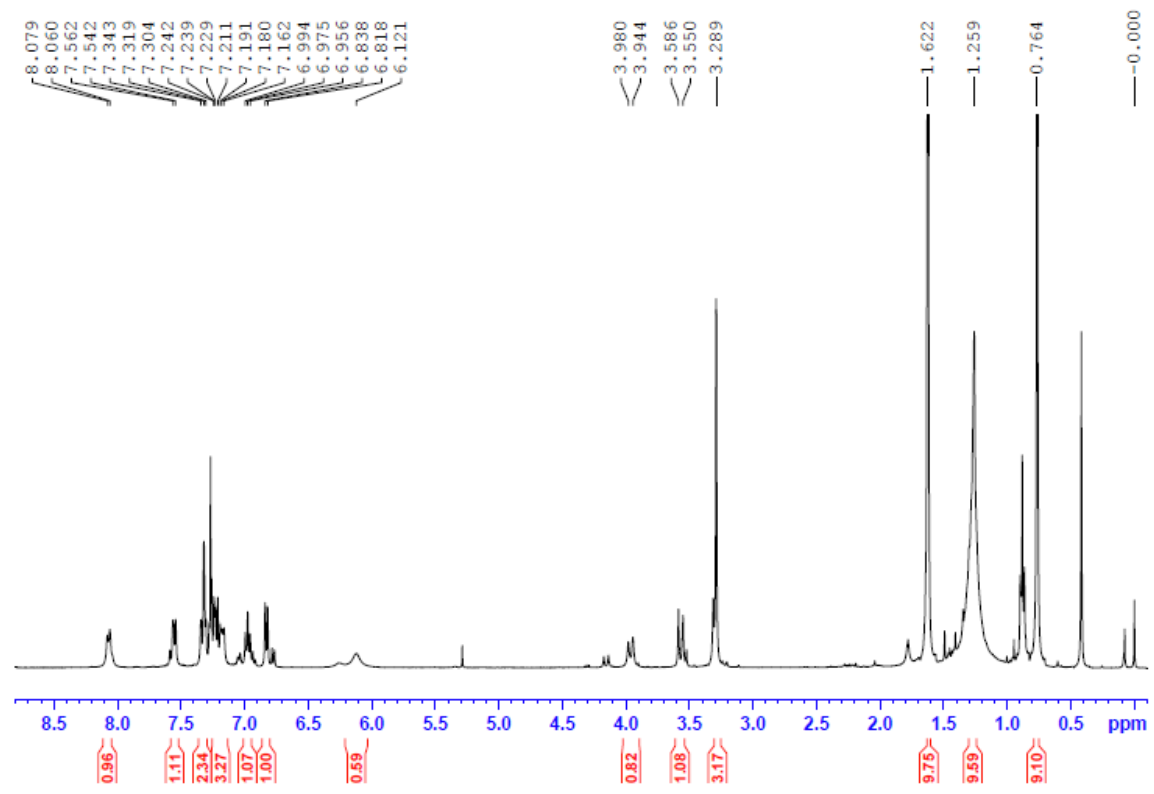

$\mathrm{CDCl}_{3},{ }^{13} \mathrm{C}\{1 \mathrm{H}\} \mathrm{NMR}, 100 \mathrm{MHz}$

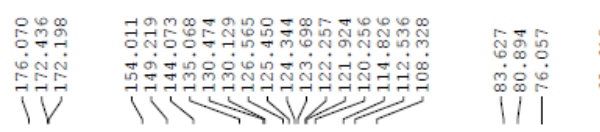

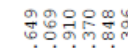

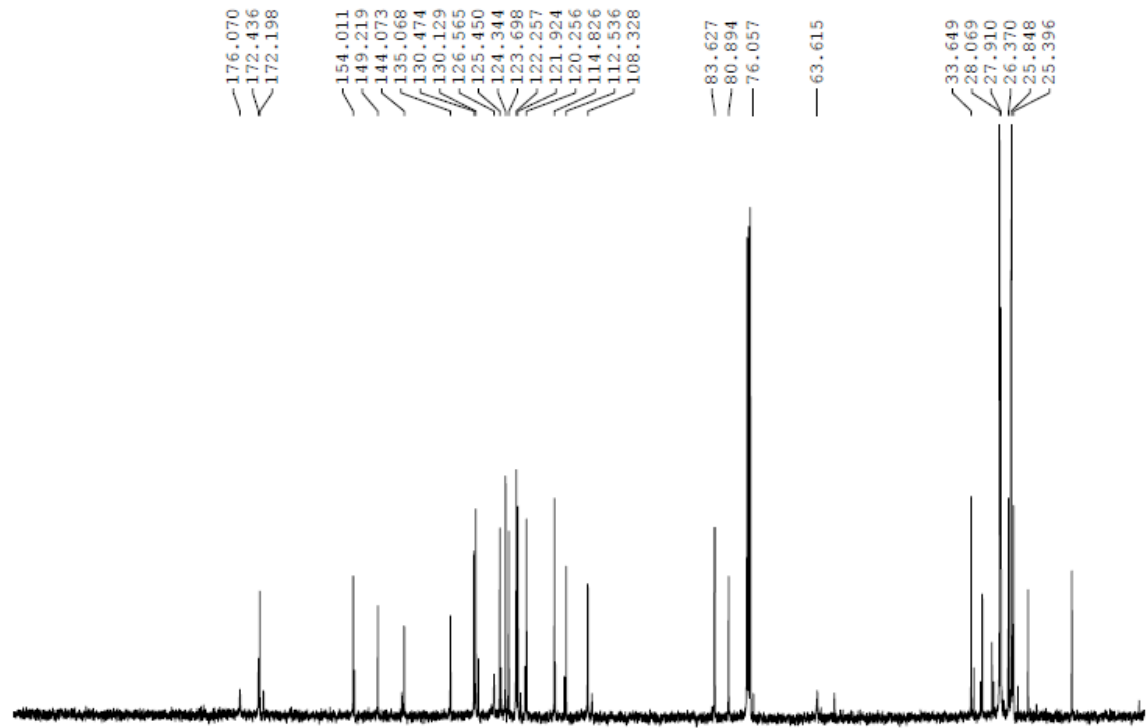

$\begin{array}{lllllllllllllllllllll}210 & 200 & 190 & 180 & 170 & 160 & 150 & 140 & 130 & 120 & 110 & 100 & 90 & 80 & 70 & 60 & 50 & 40 & 30 & 20 & \text { ppm }\end{array}$ 


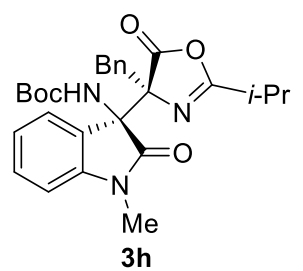

$\mathrm{CDCl}_{3},{ }^{1} \mathrm{H} \mathrm{NMR}, 400 \mathrm{MHz}$

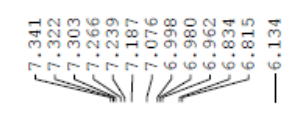

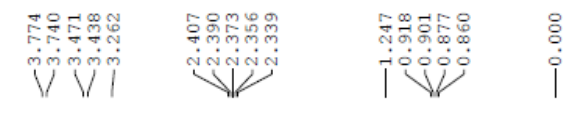

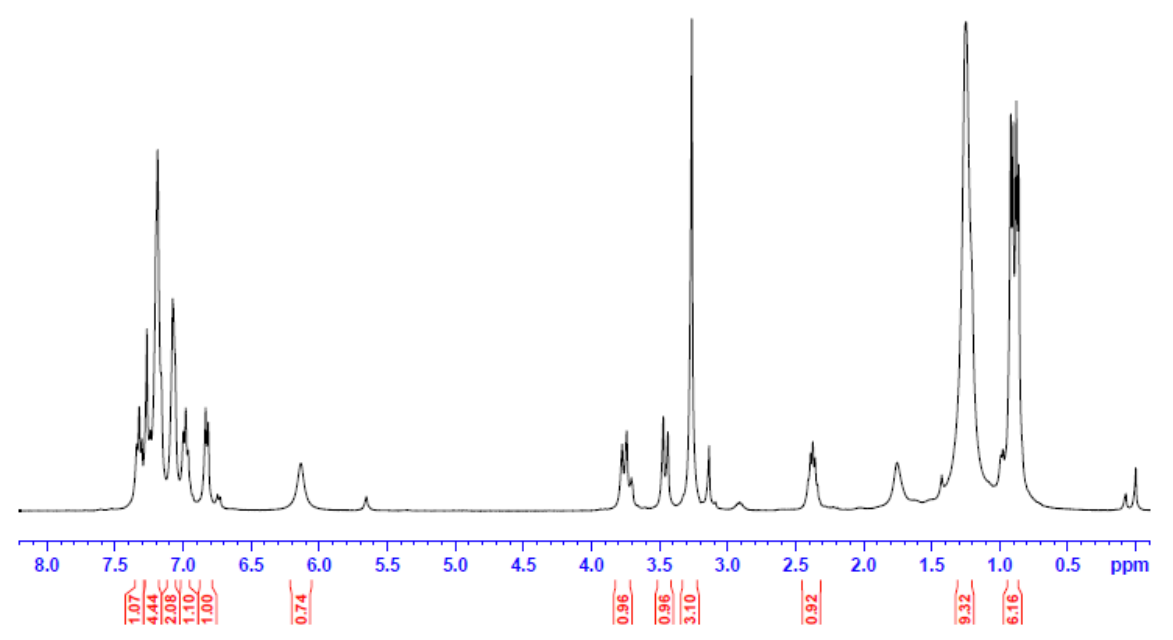

$\mathrm{CDCl}_{3},{ }^{13} \mathrm{C}\{1 \mathrm{H}\} \mathrm{NMR}, 100 \mathrm{MHz}$

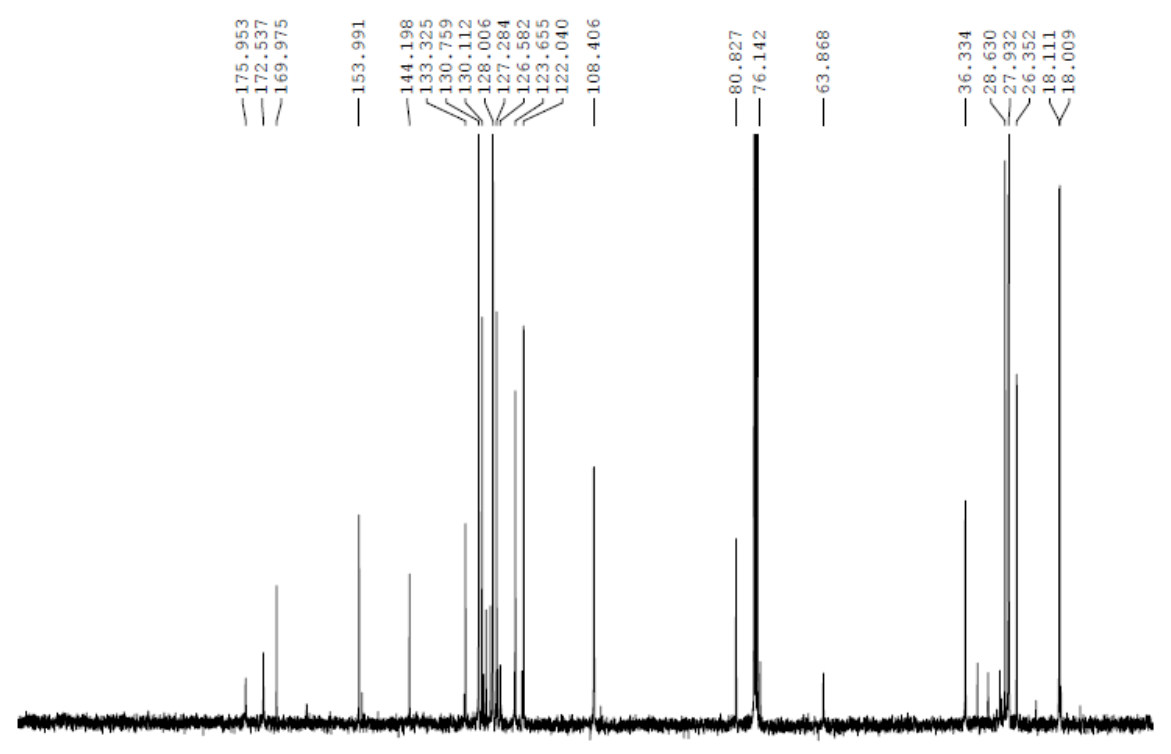

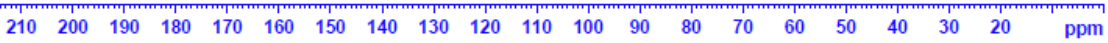




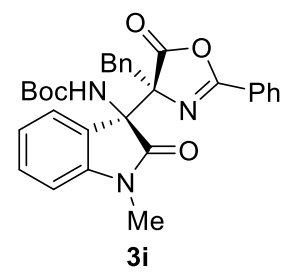

$\mathrm{CDCl}_{3},{ }^{1} \mathrm{H} \mathrm{NMR}, 400 \mathrm{MHz}$

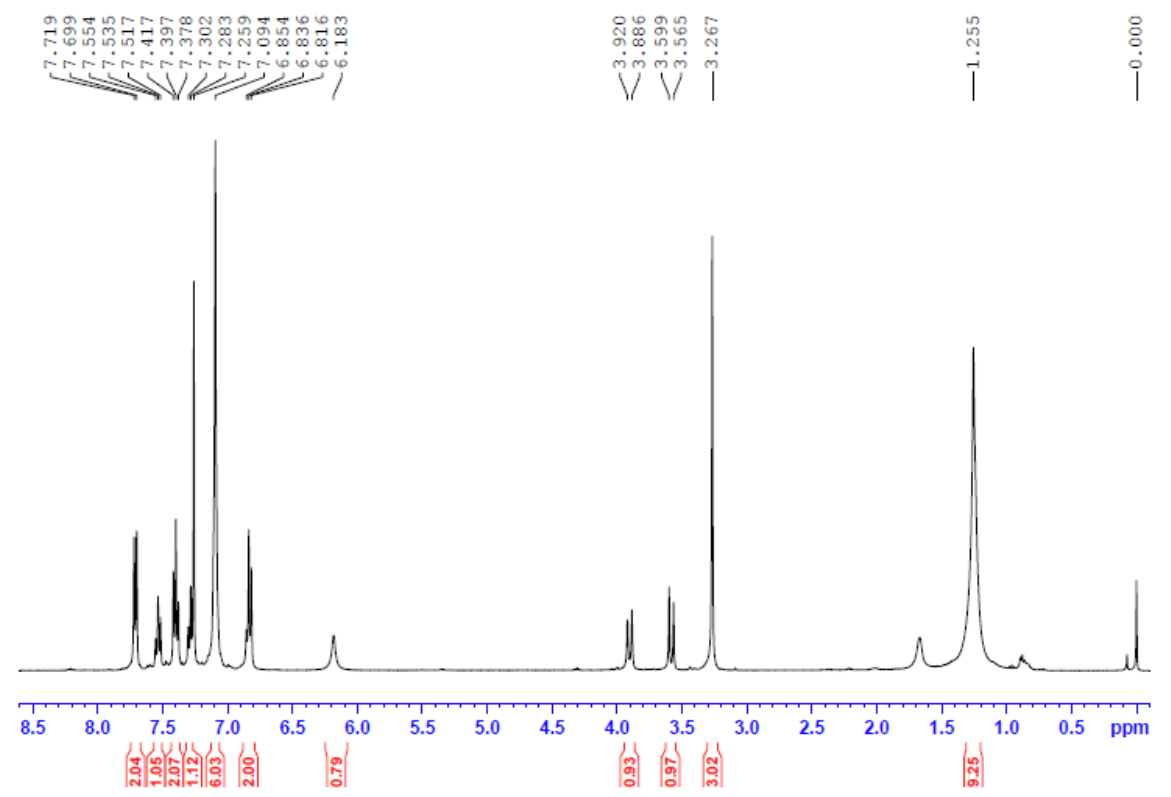

$\mathrm{CDCl}_{3},{ }^{13} \mathrm{C}\{1 \mathrm{H}\} \mathrm{NMR}, 100 \mathrm{MHz}$

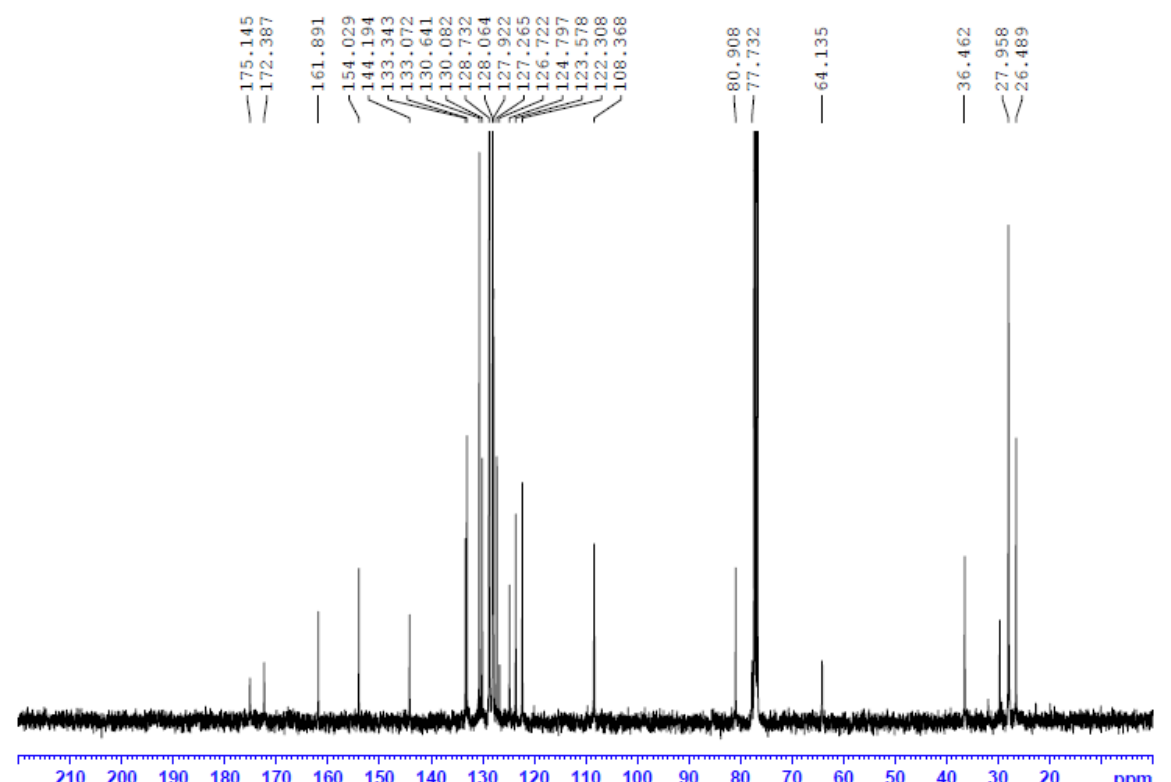

$\begin{array}{lllllllllllllllllllll}210 & 200 & 190 & 180 & 170 & 160 & 150 & 140 & 130 & 120 & 110 & 100 & 90 & 80 & 70 & 60 & 50 & 40 & 30 & 20 & \mathrm{ppm}\end{array}$ 


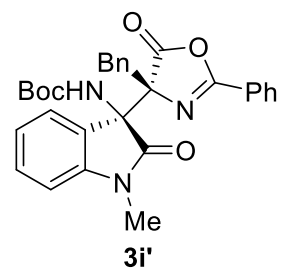

$\mathrm{CDCl}_{3},{ }^{1} \mathrm{H} \mathrm{NMR}, 400 \mathrm{MHz}$

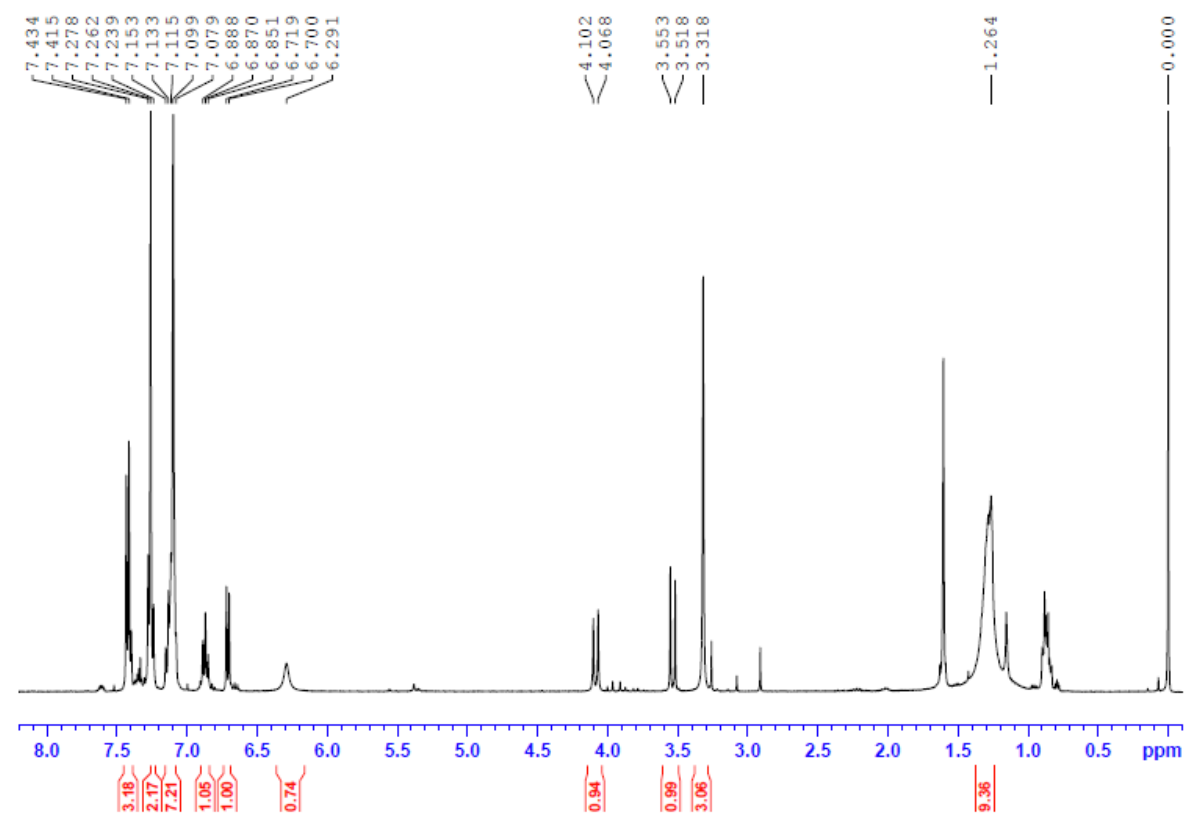

$\mathrm{CDCl}_{3},{ }^{13} \mathrm{C}\{1 \mathrm{H}\} \mathrm{NMR}, 100 \mathrm{MHz}$

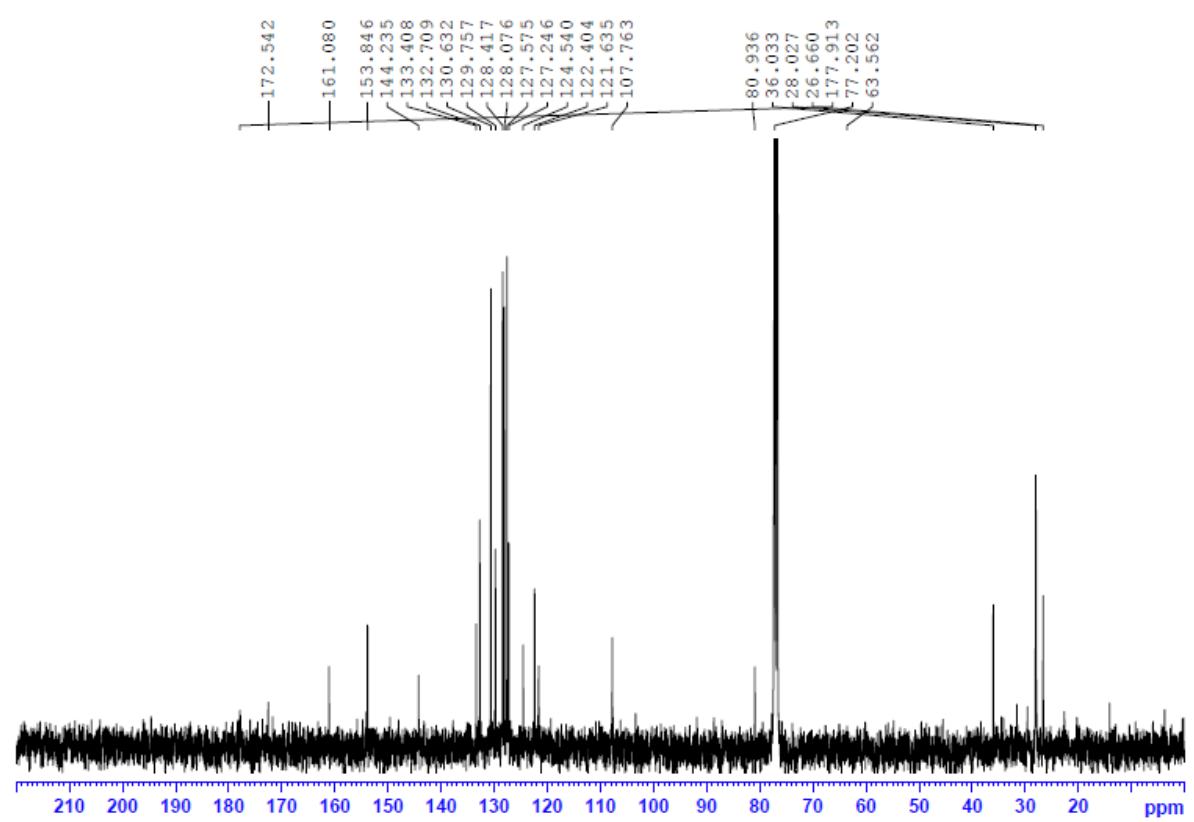




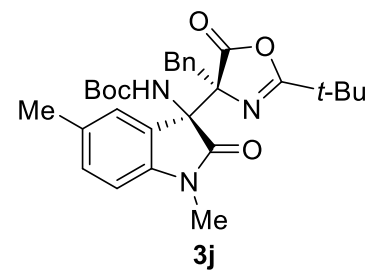

$\mathrm{CDCl}_{3},{ }^{1} \mathrm{H} \mathrm{NMR}, 400 \mathrm{MHz}$

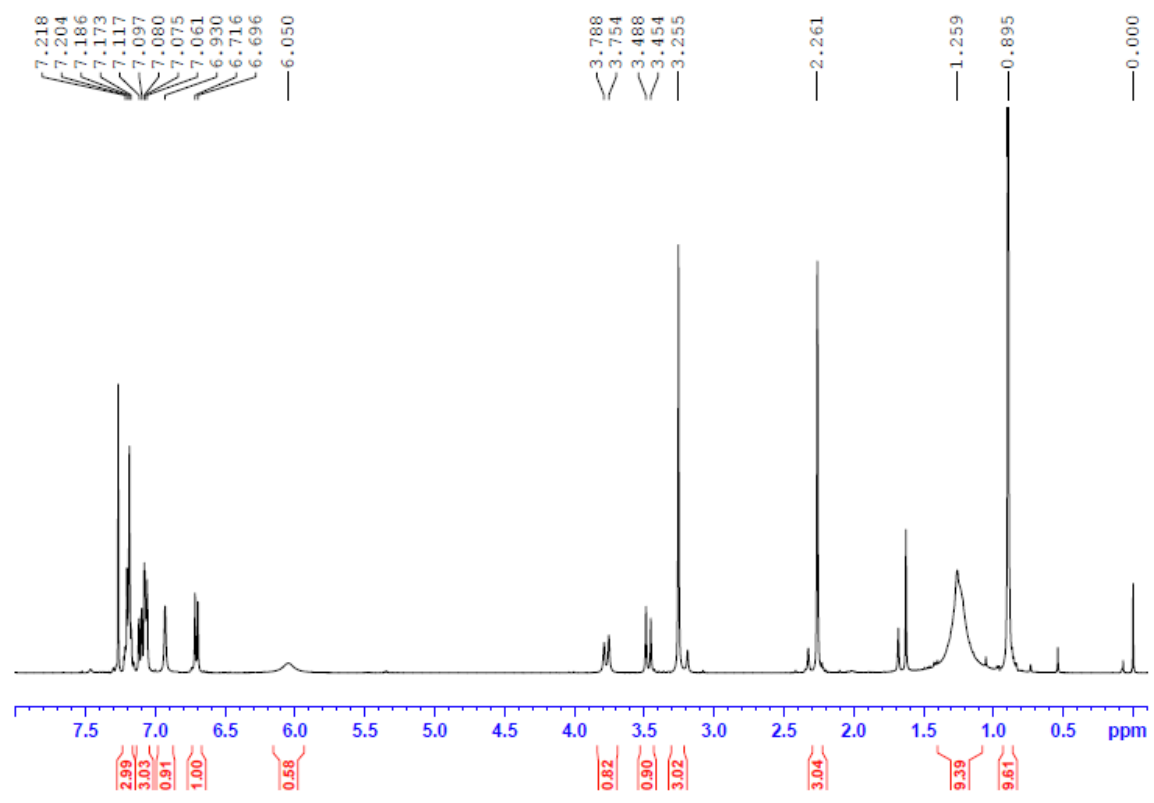

$\mathrm{CDCl}_{3},{ }^{13} \mathrm{C}\{1 \mathrm{H}\} \mathrm{NMR}, 100 \mathrm{MHz}$

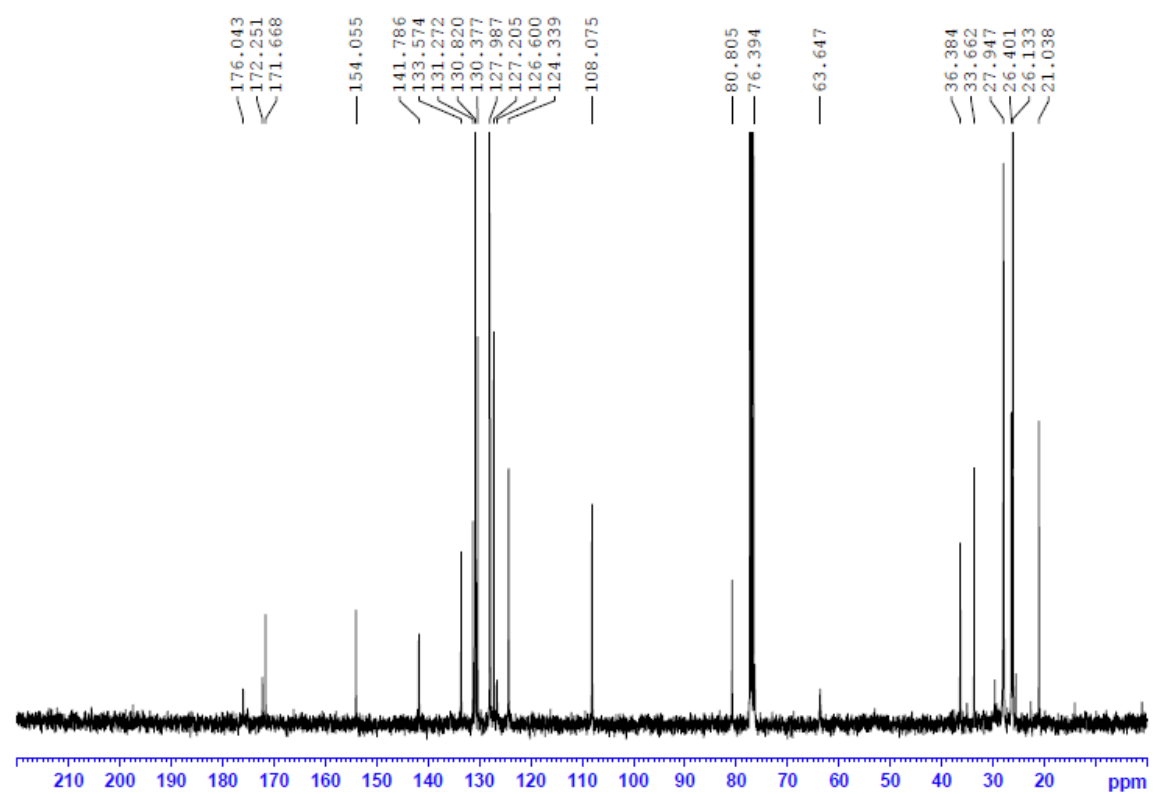




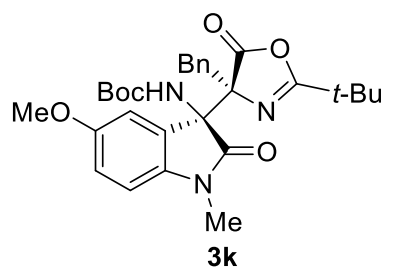

$\mathrm{CDCl}_{3},{ }^{1} \mathrm{H} \mathrm{NMR}, 400 \mathrm{MHz}$

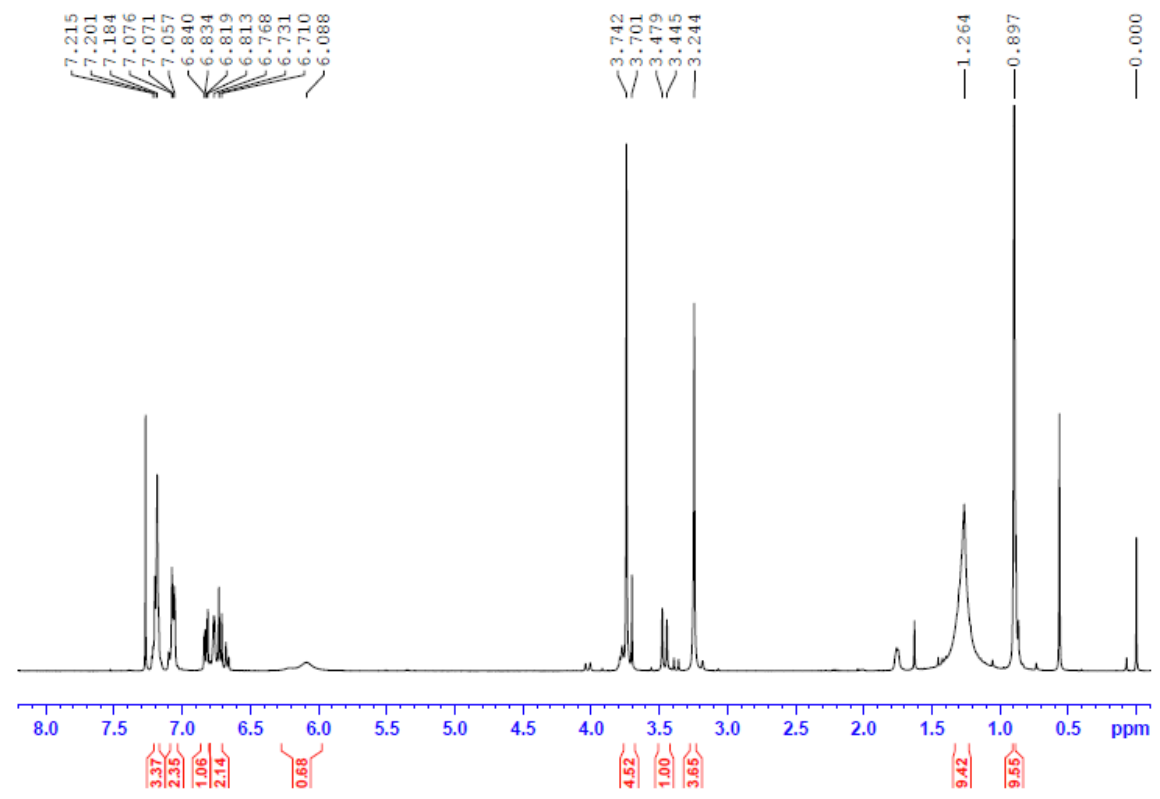

$\mathrm{CDCl}_{3},{ }^{13} \mathrm{C}\{1 \mathrm{H}\} \mathrm{NMR}, 100 \mathrm{MHz}$

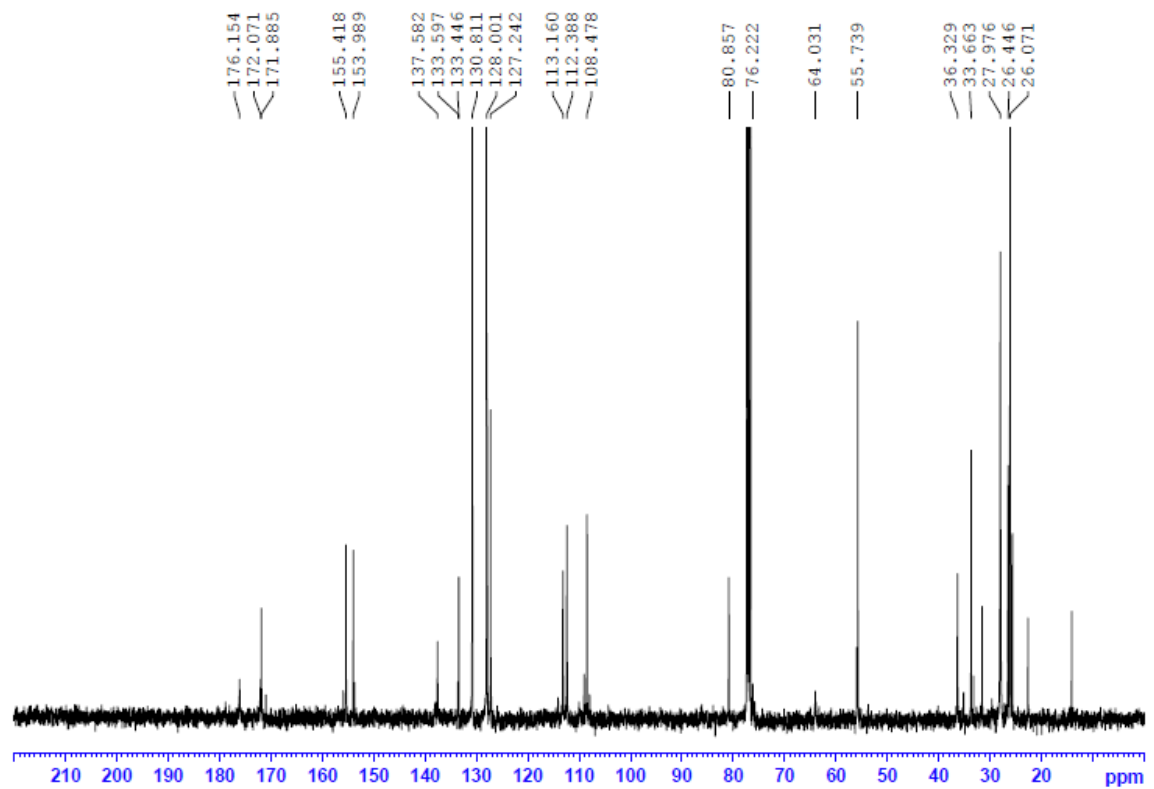




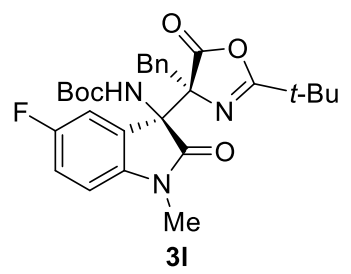

$\mathrm{CDCl}_{3},{ }^{1} \mathrm{H} \mathrm{NMR}, 400 \mathrm{MHz}$

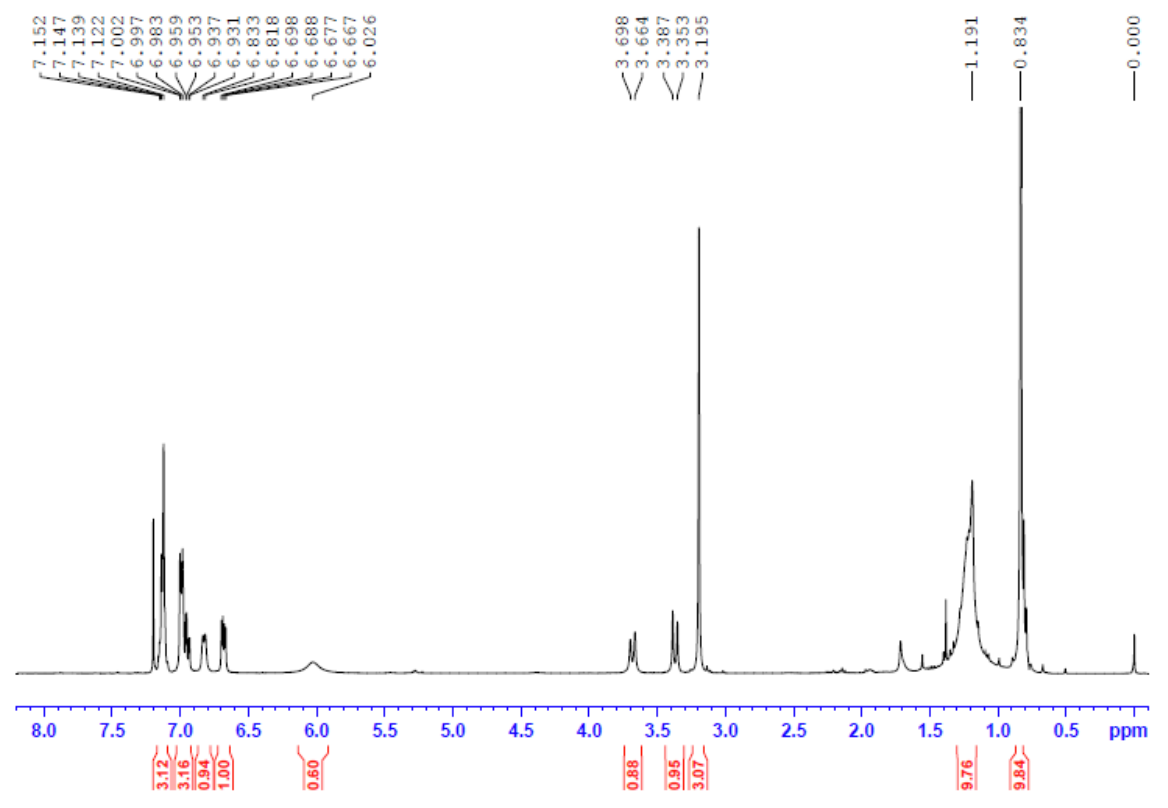

$\mathrm{CDCl}_{3},{ }^{13} \mathrm{C}\{1 \mathrm{H}\} \mathrm{NMR}, 100 \mathrm{MHz}$

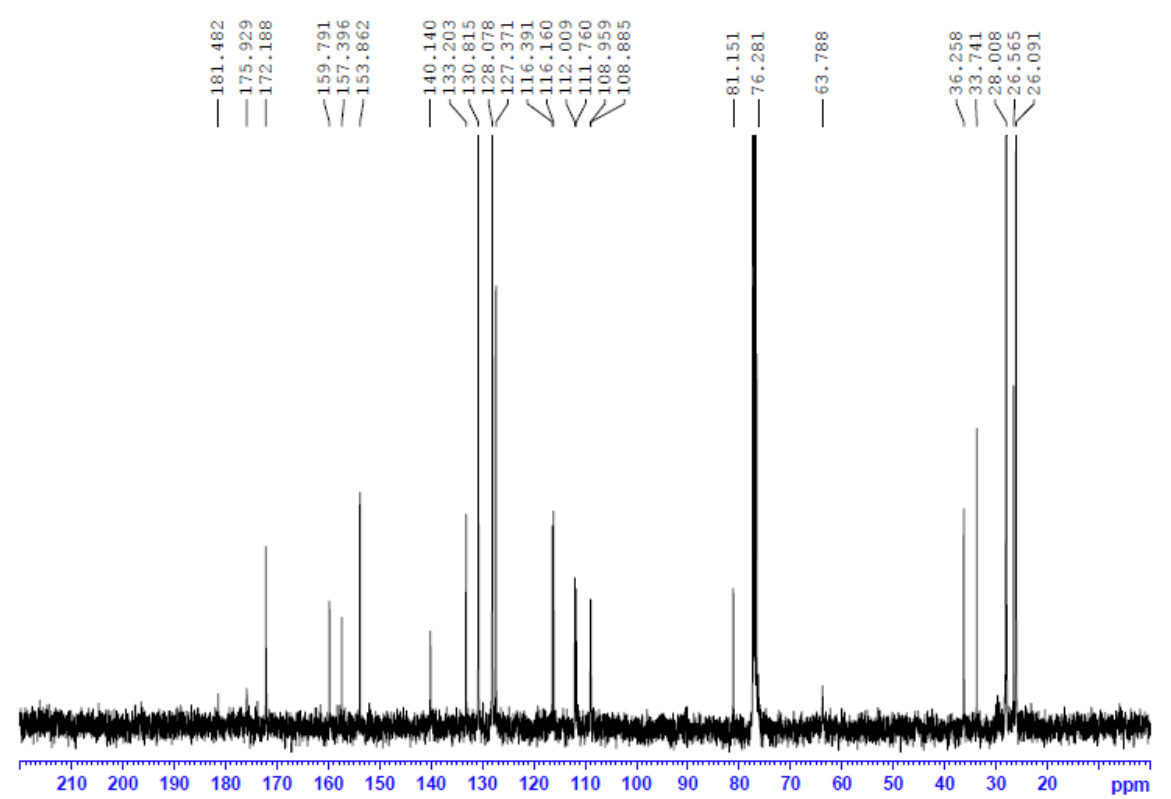




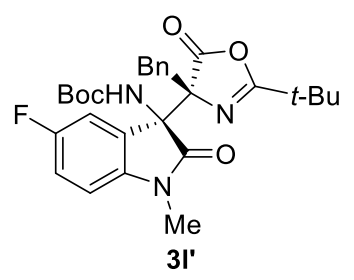

$\mathrm{CDCl}_{3},{ }^{1} \mathrm{H} \mathrm{NMR}, 400 \mathrm{MHz}$

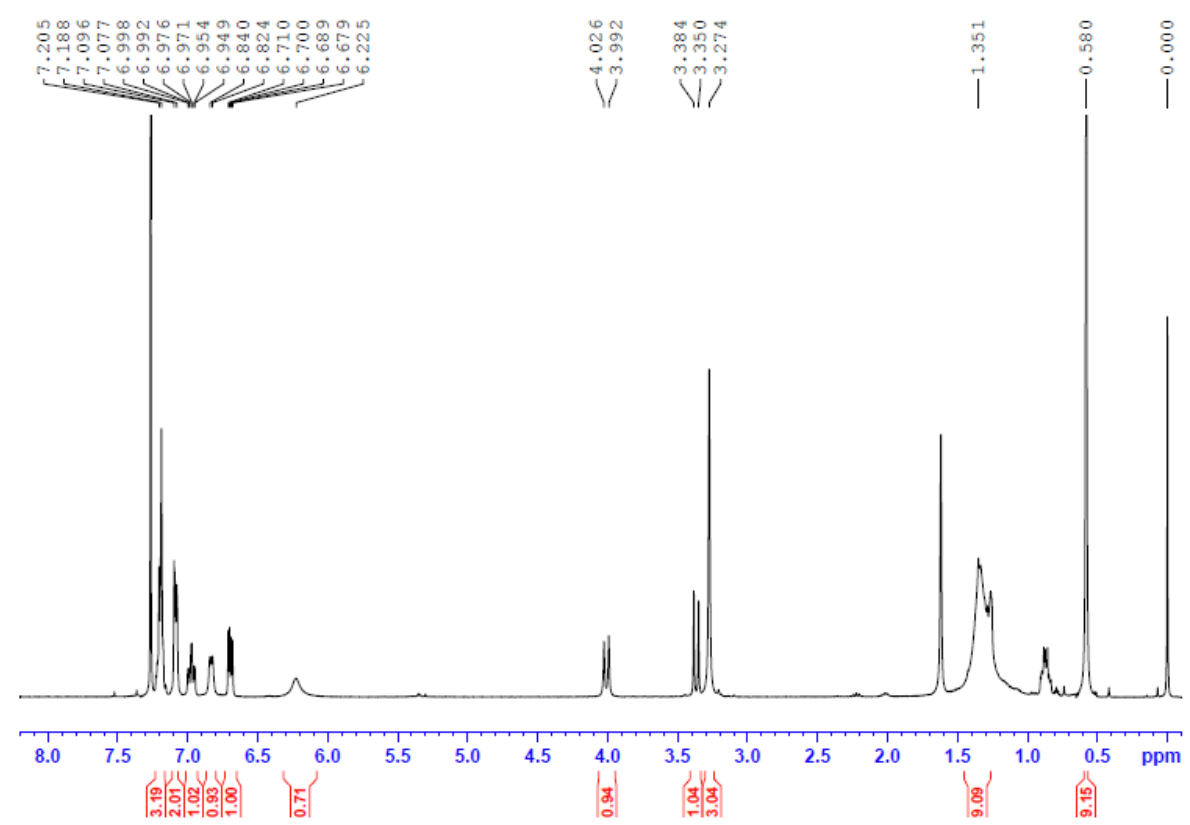

$\mathrm{CDCl}_{3},{ }^{13} \mathrm{C}\{1 \mathrm{H}\} \mathrm{NMR}, 100 \mathrm{MHz}$

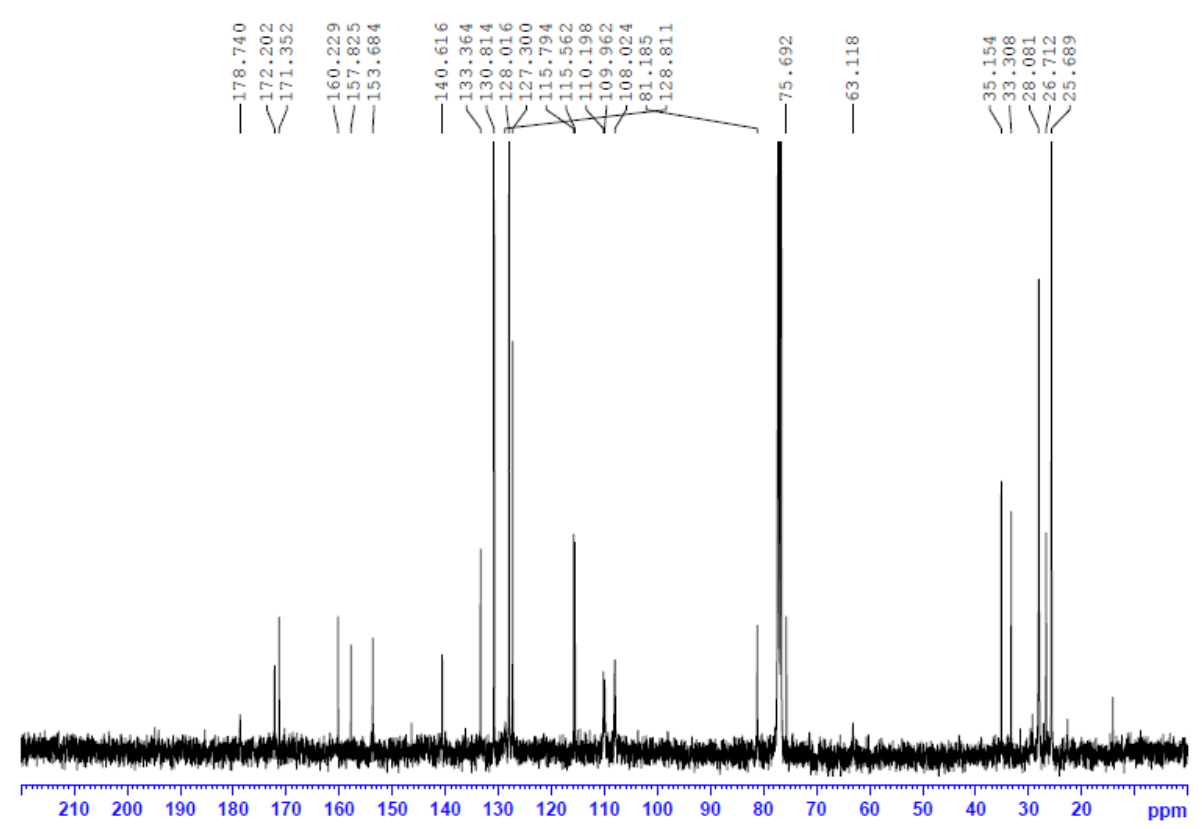




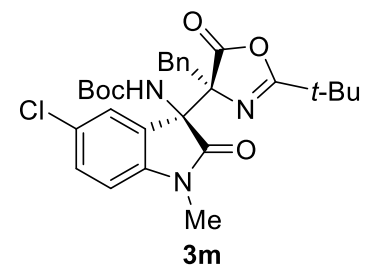

$\mathrm{CDCl}_{3},{ }^{1} \mathrm{H} \mathrm{NMR}, 400 \mathrm{MHz}$

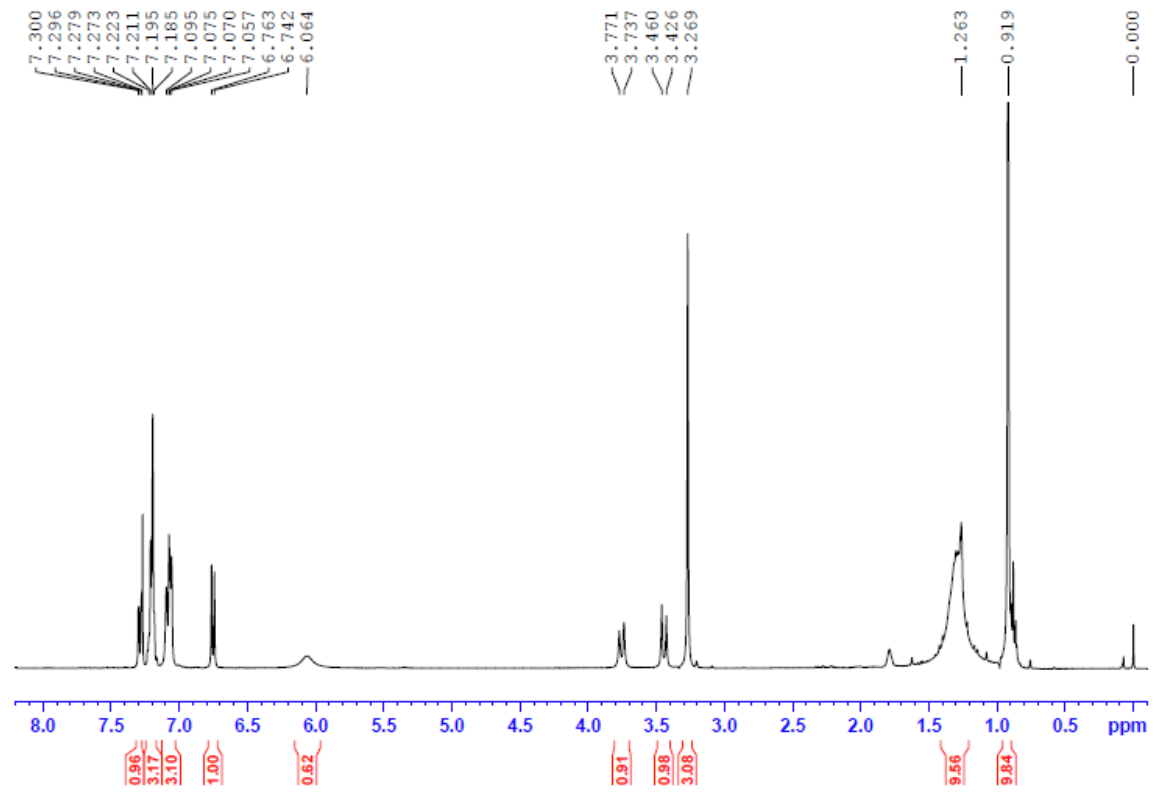

$\mathrm{CDCl}_{3},{ }^{13} \mathrm{C}\{1 \mathrm{H}\} \mathrm{NMR}, 100 \mathrm{MHz}$

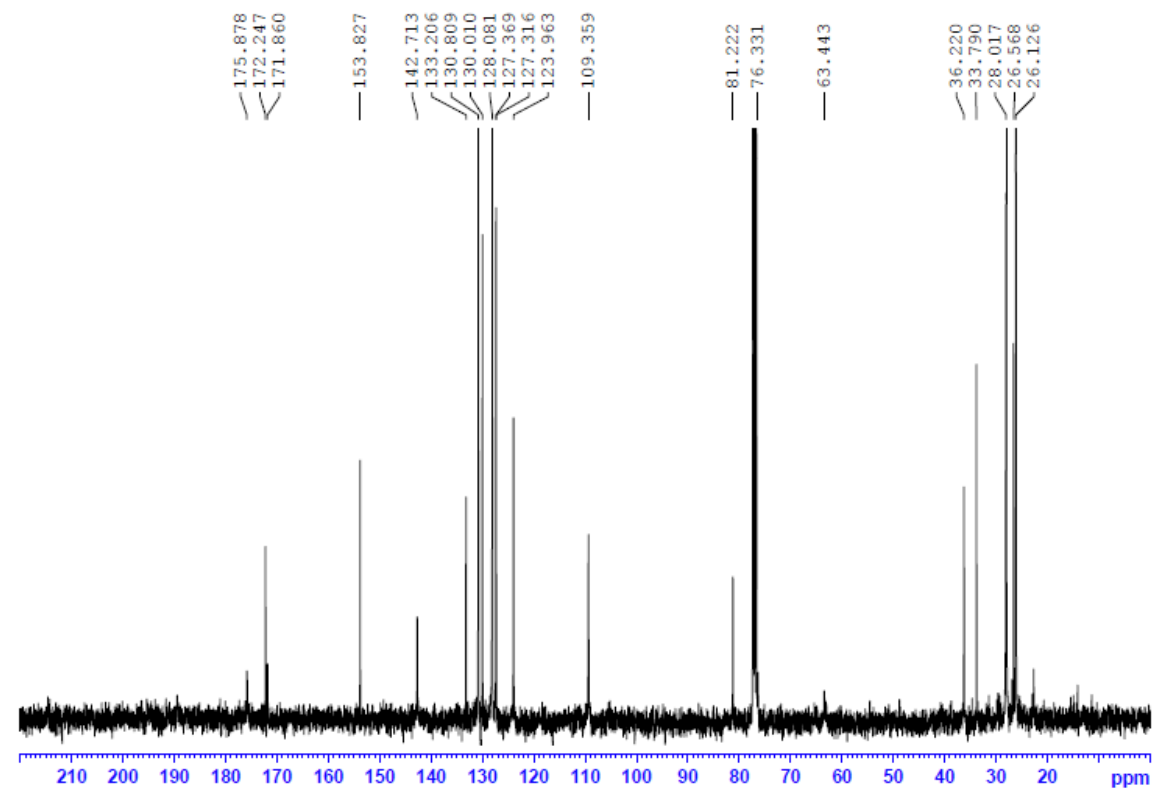




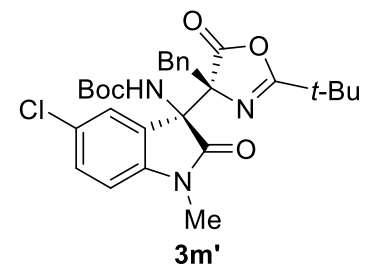

$\mathrm{CDCl}_{3},{ }^{1} \mathrm{H} \mathrm{NMR}, 400 \mathrm{MHz}$

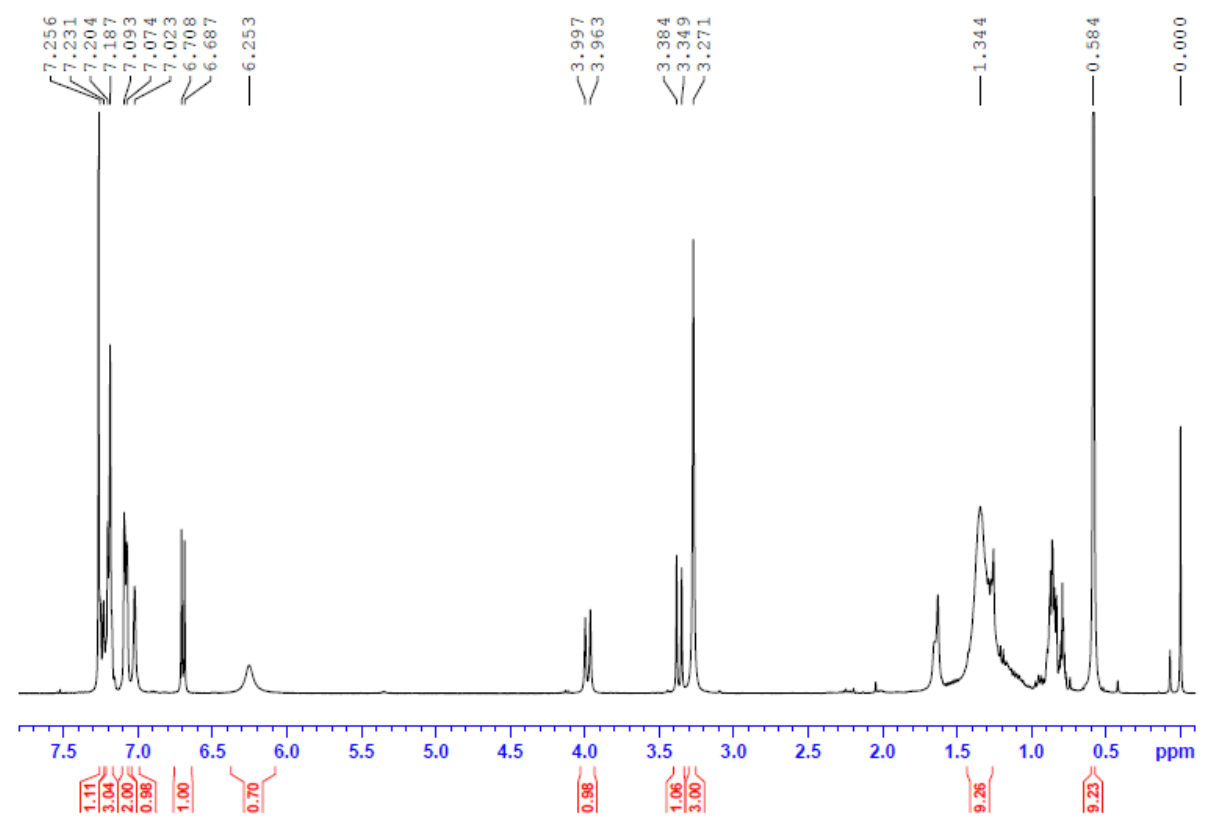

$\mathrm{CDCl}_{3},{ }^{13} \mathrm{C}\{1 \mathrm{H}\} \mathrm{NMR}, 100 \mathrm{MHz}$

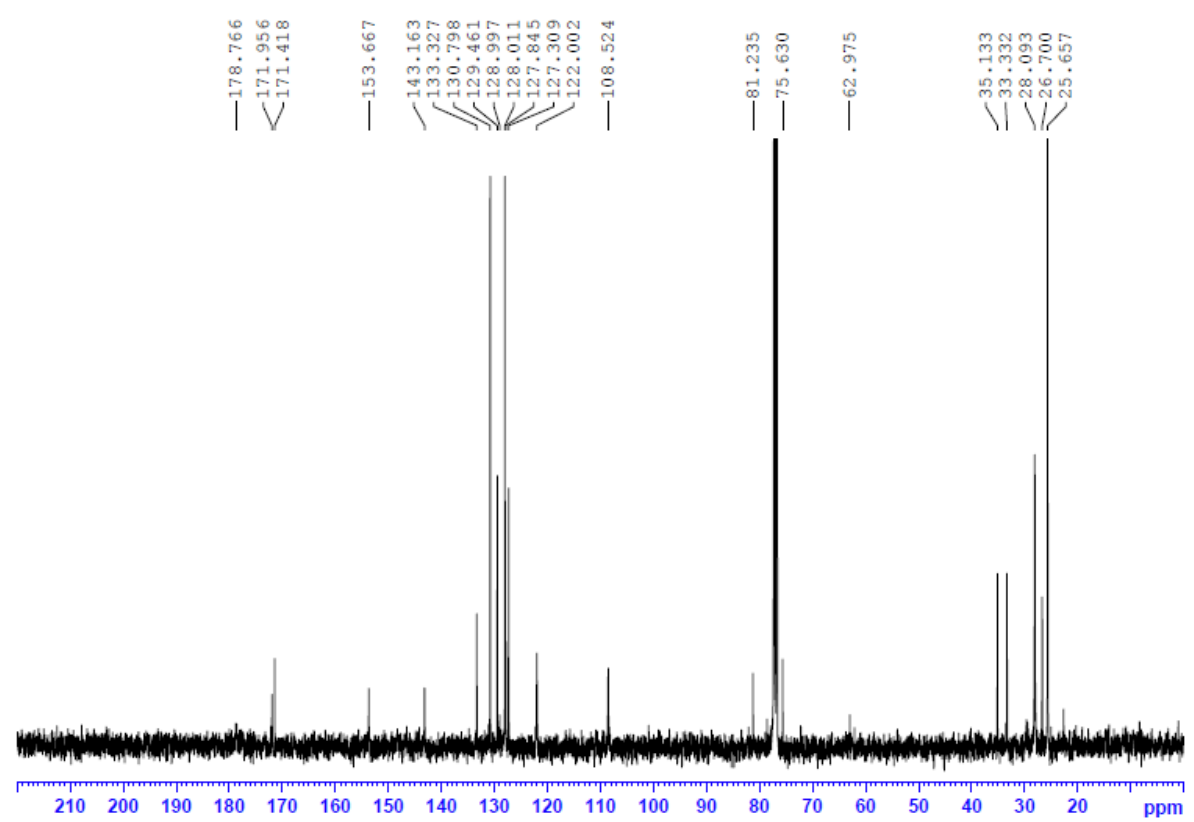




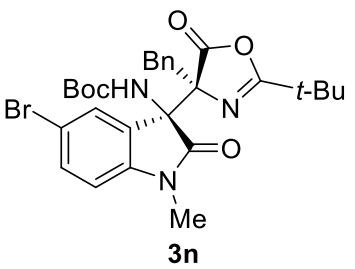

$\mathrm{CDCl}_{3},{ }^{1} \mathrm{H} \mathrm{NMR}, 400 \mathrm{MHz}$

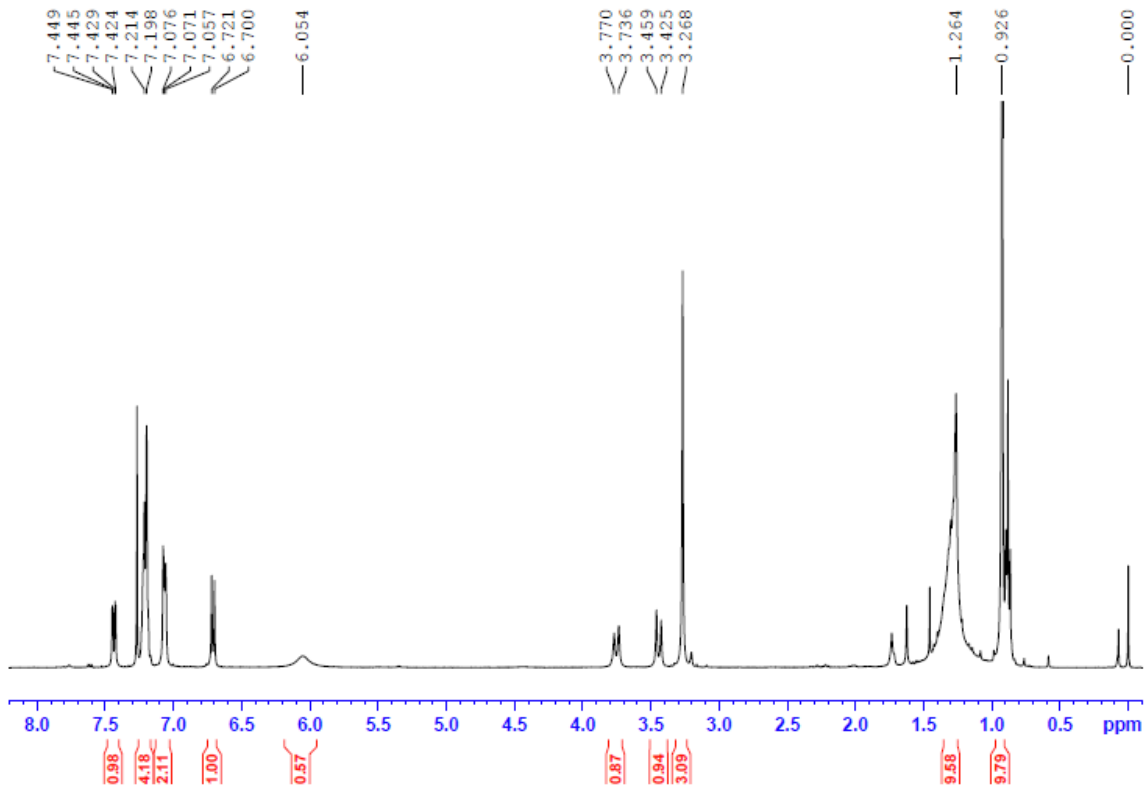

$\mathrm{CDCl}_{3},{ }^{13} \mathrm{C}\{1 \mathrm{H}\} \mathrm{NMR}, 100 \mathrm{MHz}$

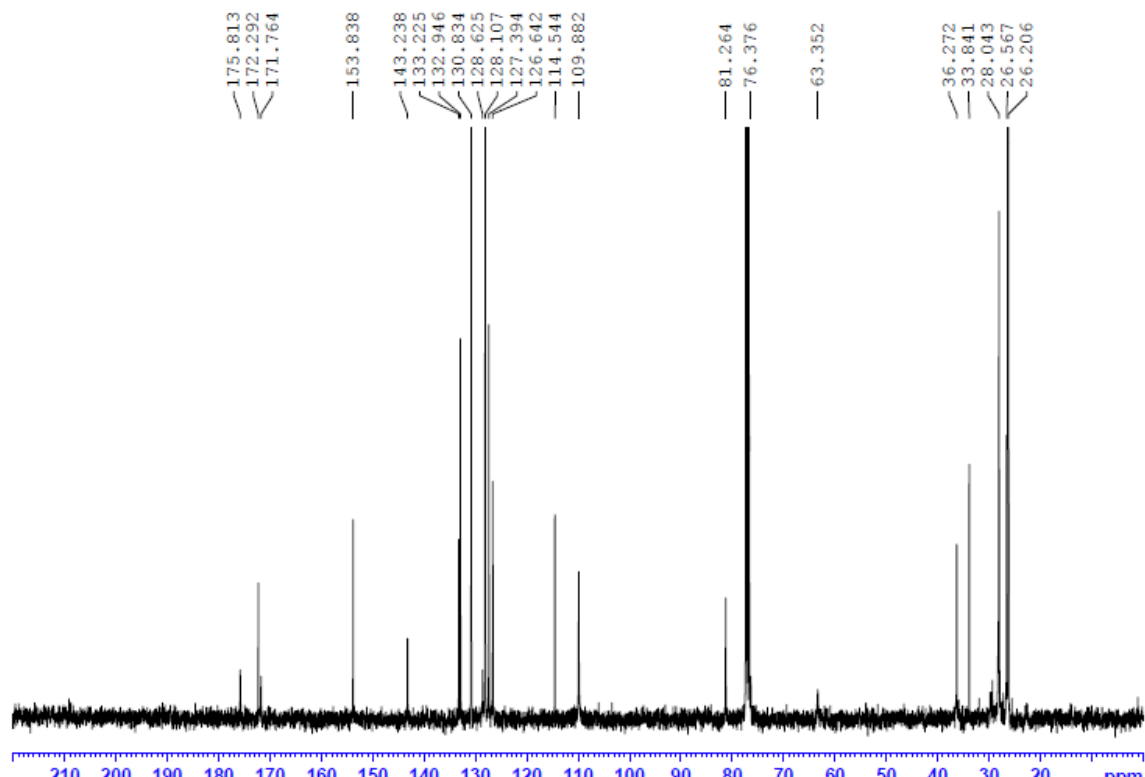

$\begin{array}{lllllllllllllllllllll}210 & 200 & 190 & 180 & 170 & 160 & 150 & 140 & 130 & 120 & 110 & 100 & 90 & 80 & 70 & 60 & 50 & 40 & 30 & 20 & \text { ppm }\end{array}$ 


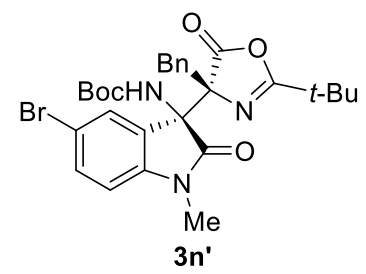

$\mathrm{CDCl}_{3},{ }^{1} \mathrm{H} \mathrm{NMR}, 400 \mathrm{MHz}$

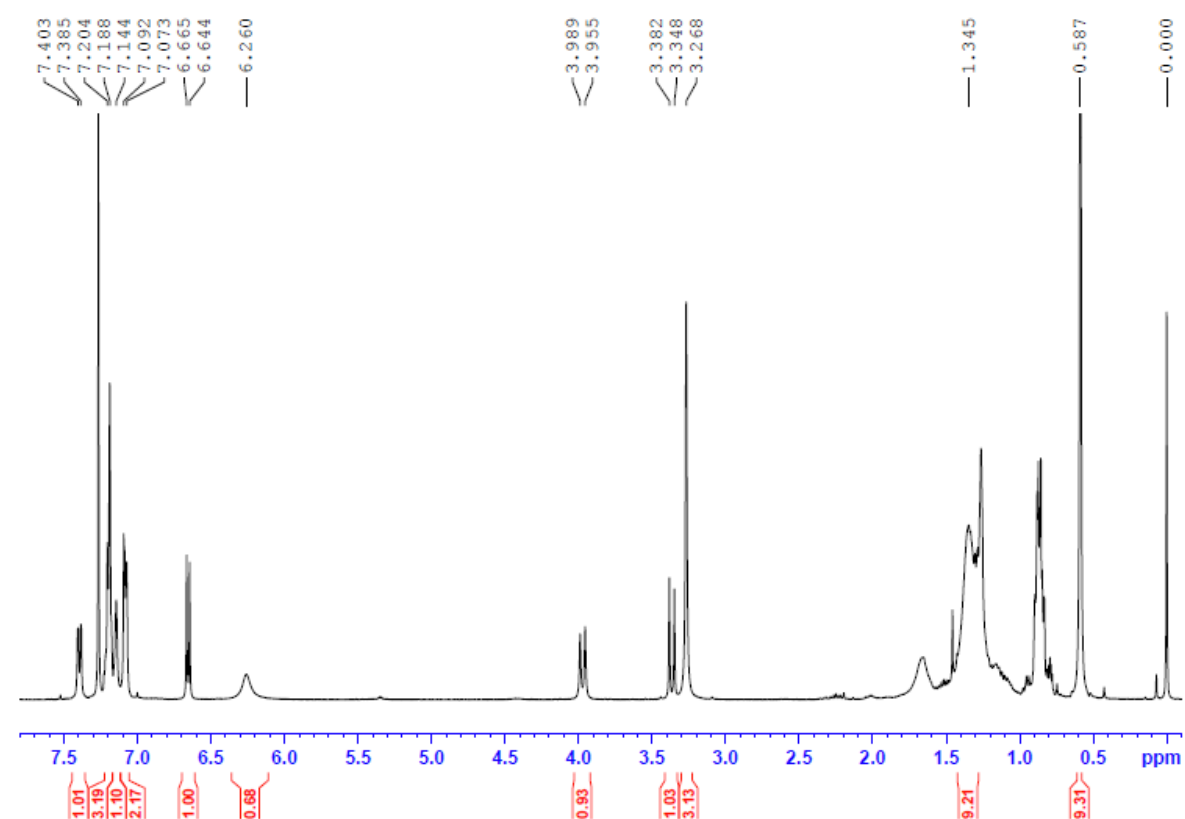

$\mathrm{CDCl}_{3},{ }^{13} \mathrm{C}\{1 \mathrm{H}\} \mathrm{NMR}, 100 \mathrm{MHz}$

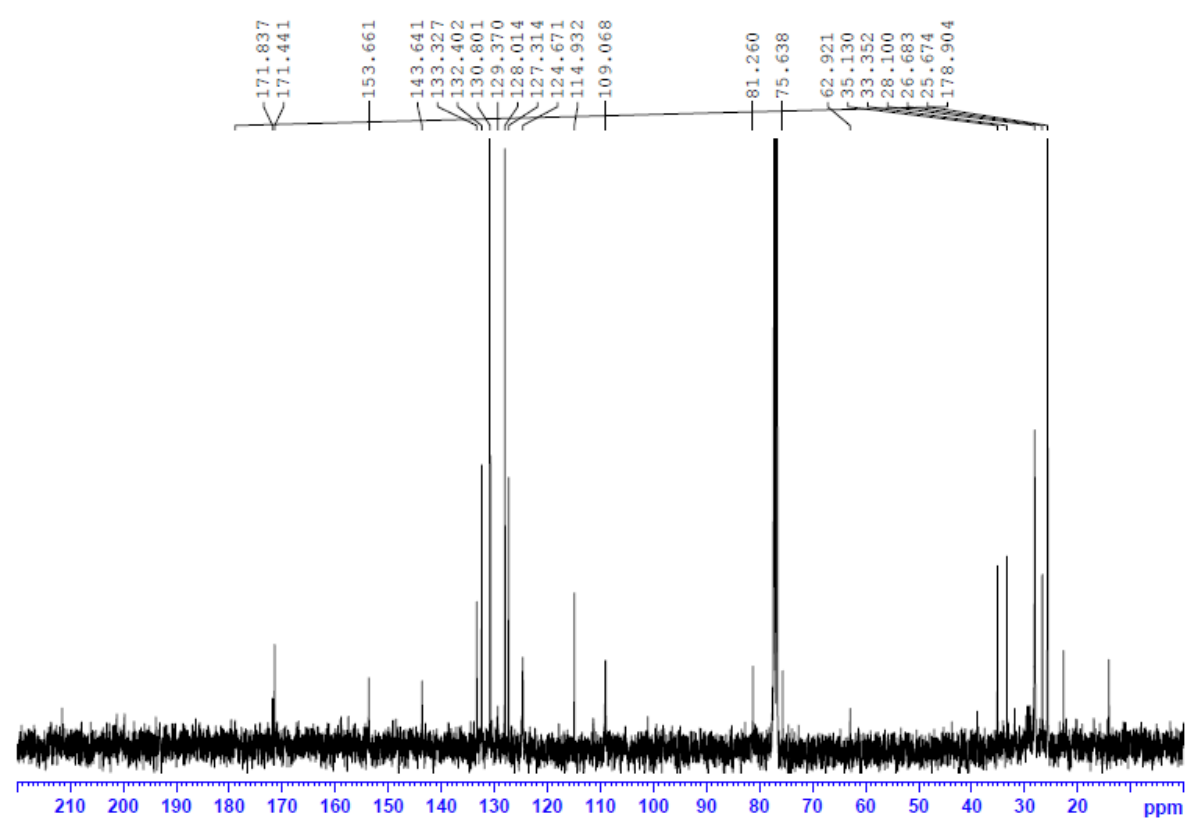




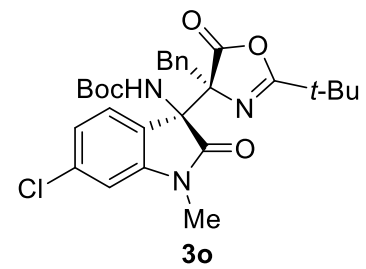

$\mathrm{CDCl}_{3},{ }^{1} \mathrm{H} \mathrm{NMR}, 400 \mathrm{MHz}$

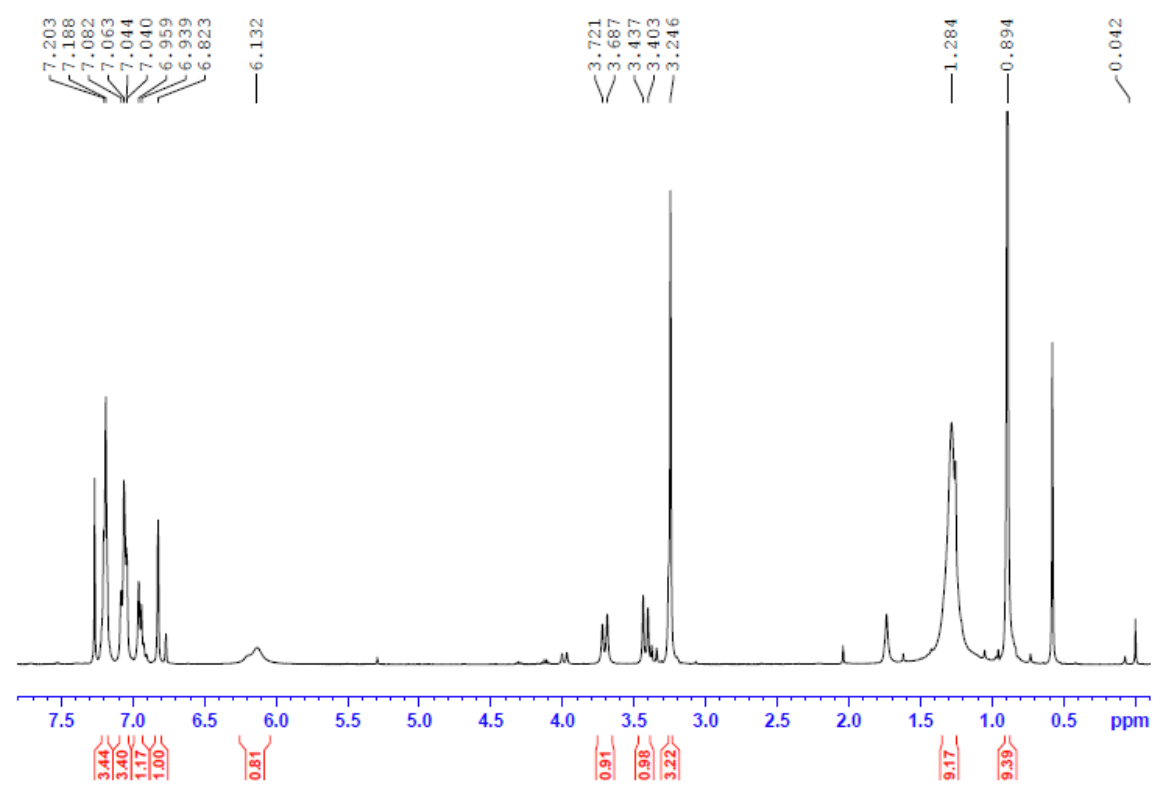

$\mathrm{CDCl}_{3},{ }^{13} \mathrm{C}\{1 \mathrm{H}\} \mathrm{NMR}, 100 \mathrm{MHz}$

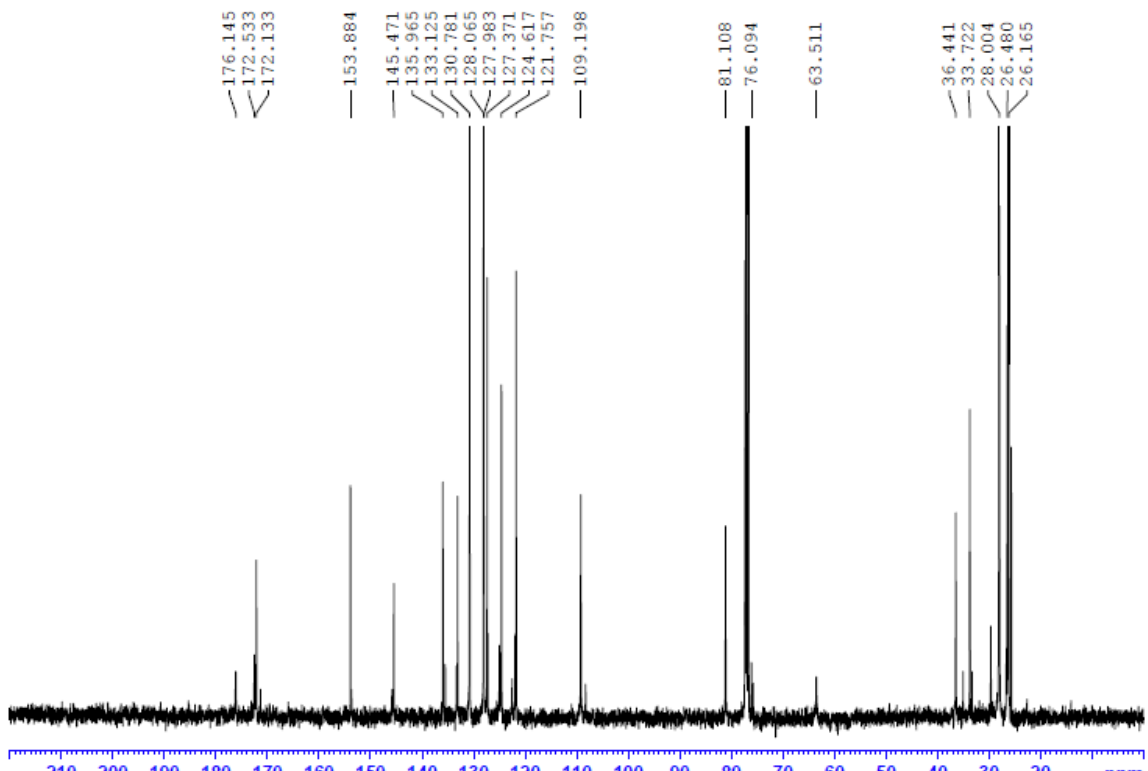

$\begin{array}{lllllllllllllllllllll}210 & 200 & 190 & 180 & 170 & 160 & 150 & 140 & 130 & 120 & 110 & 100 & 90 & 80 & 70 & 60 & 50 & 40 & 30 & 20 & \end{array}$ 


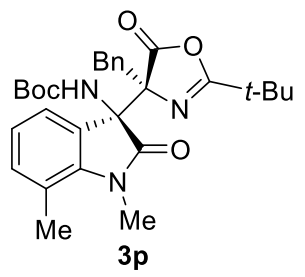

$\mathrm{CDCl}_{3},{ }^{1} \mathrm{H} \mathrm{NMR}, 400 \mathrm{MHz}$

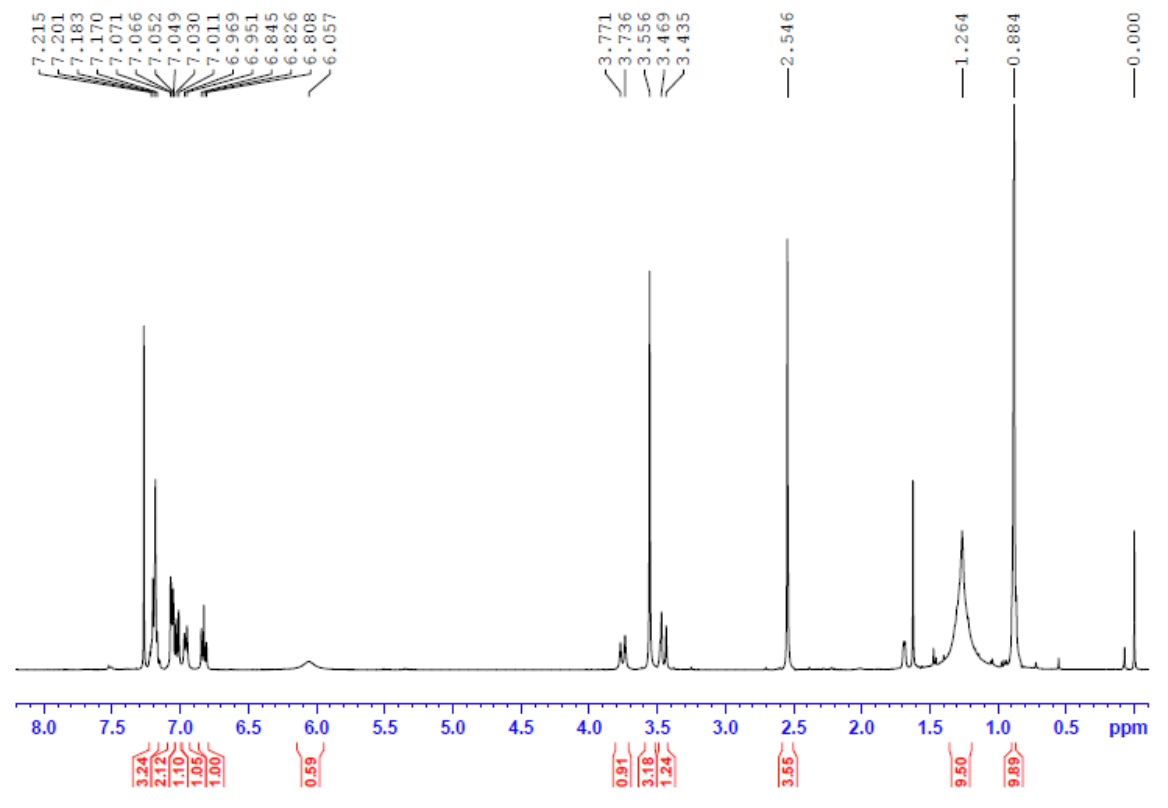

$\mathrm{CDCl}_{3},{ }^{13} \mathrm{C}\{1 \mathrm{H}\} \mathrm{NMR}, 100 \mathrm{MHz}$

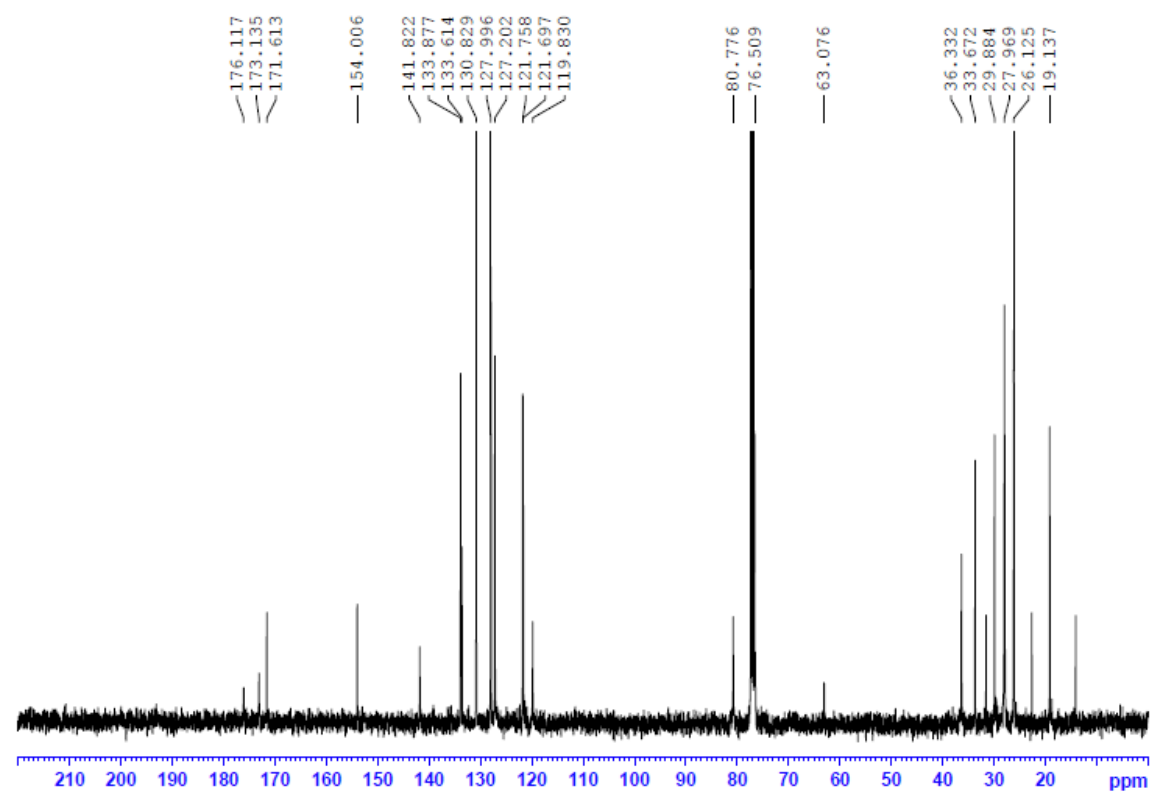




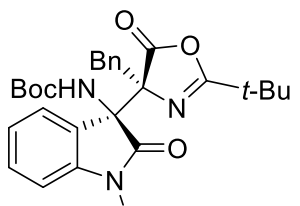

Allyl

$3 q$

$\mathrm{CDCl}_{3},{ }^{1} \mathrm{H} \mathrm{NMR}, 400 \mathrm{MHz}$

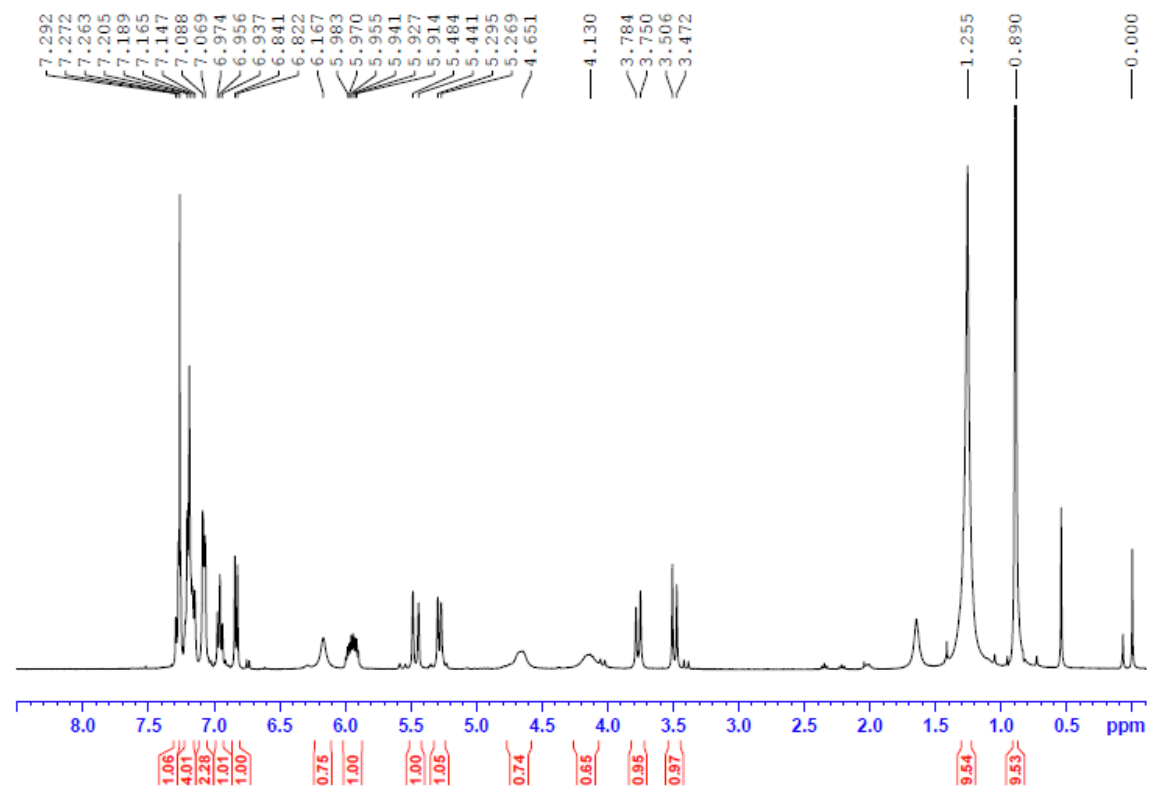

$\mathrm{CDCl}_{3},{ }^{13} \mathrm{C}\{1 \mathrm{H}\} \mathrm{NMR}, 100 \mathrm{MHz}$
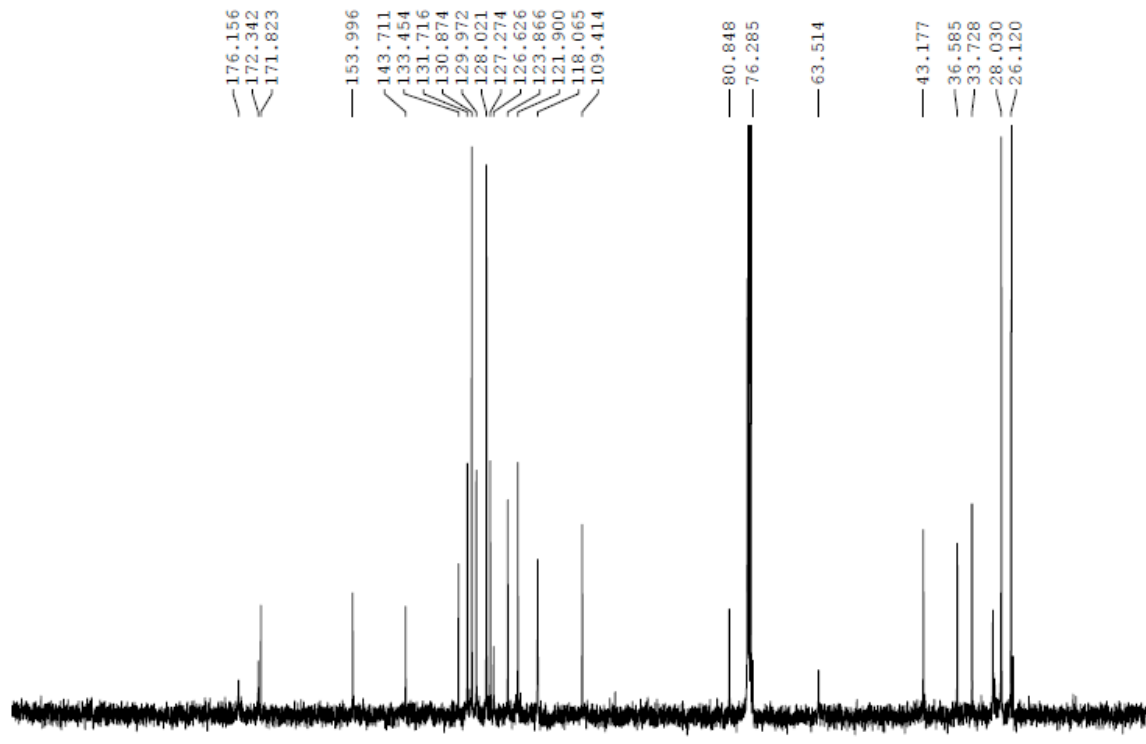

$\begin{array}{llllllllllllllllllll}210 & 200 & 190 & 180 & 170 & 160 & 150 & 140 & 130 & 120 & 110 & 100 & 90 & 80 & 70 & 60 & 50 & 40 & 30 & 20\end{array}$ 


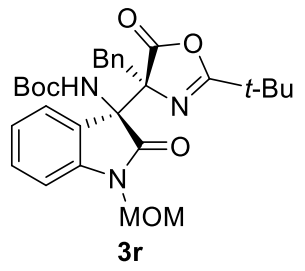

$\mathrm{CDCl}_{3},{ }^{1} \mathrm{H} \mathrm{NMR}, 400 \mathrm{MHz}$

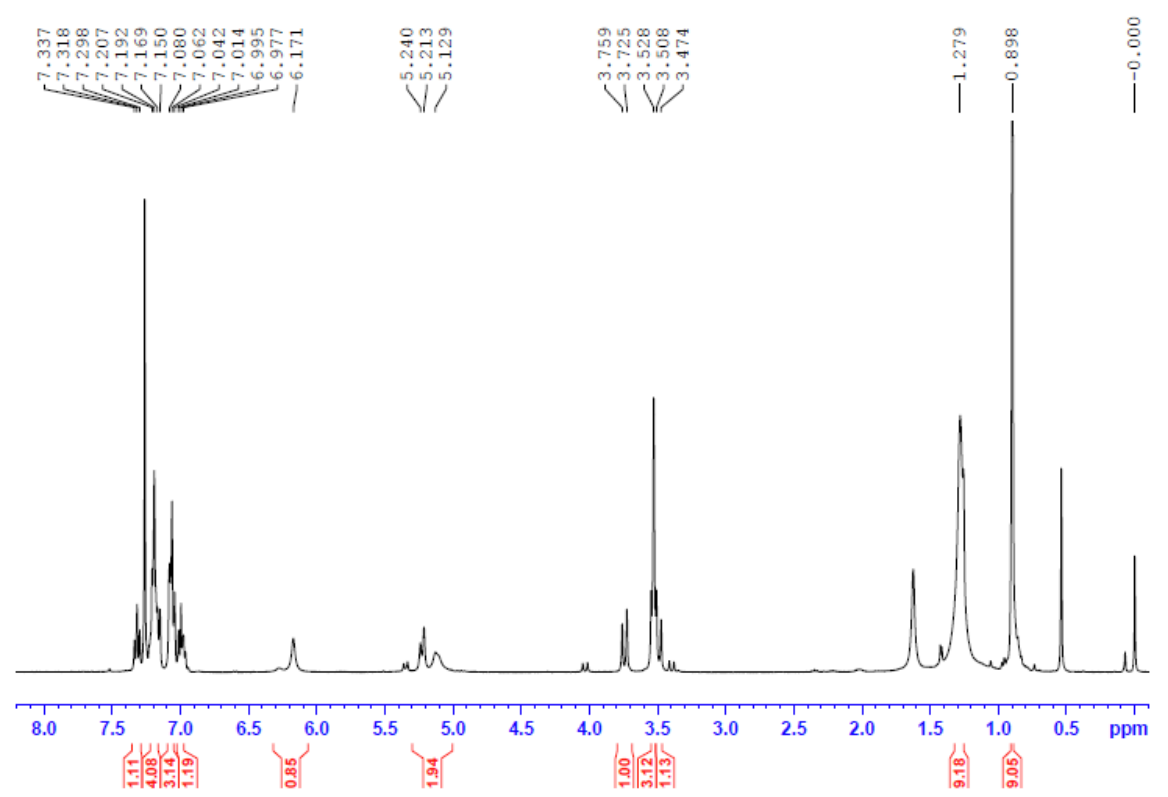

$\mathrm{CDCl}_{3},{ }^{13} \mathrm{C}\{1 \mathrm{H}\} \mathrm{NMR}, 100 \mathrm{MHz}$

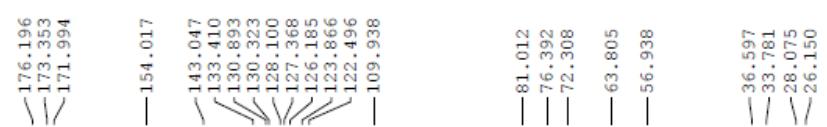

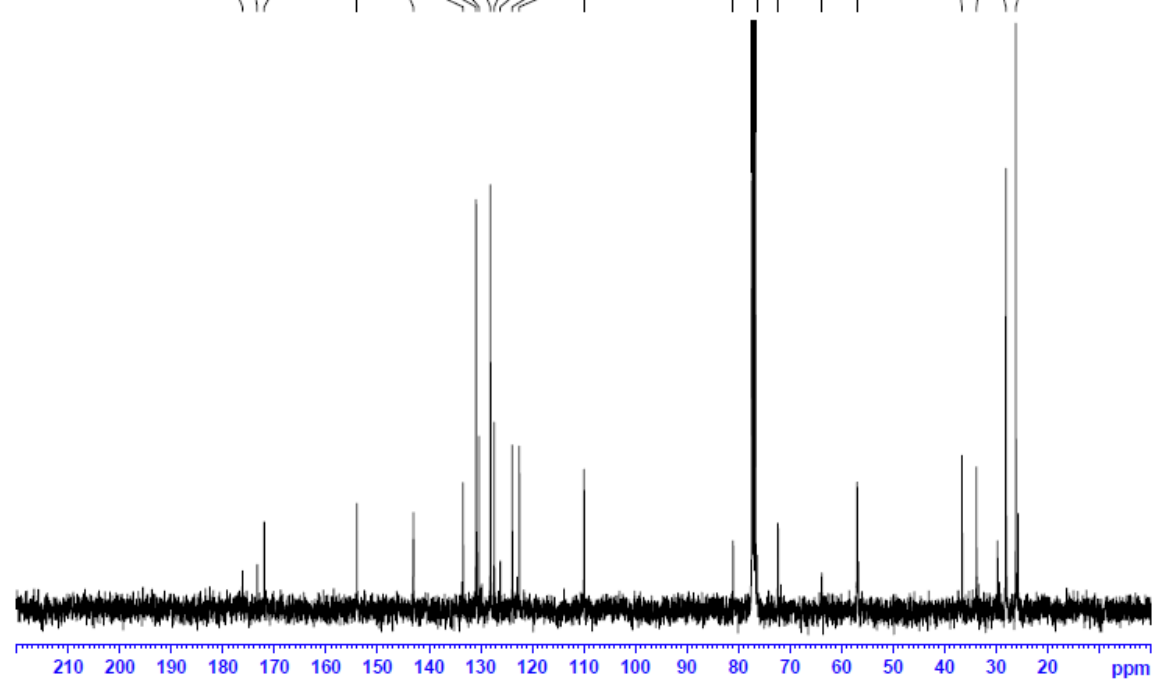




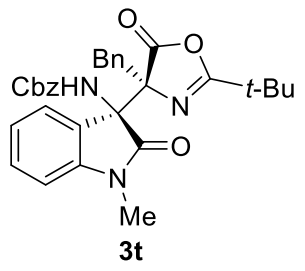

$\mathrm{CDCl}_{3},{ }^{1} \mathrm{H} \mathrm{NMR}, 400 \mathrm{MHz}$

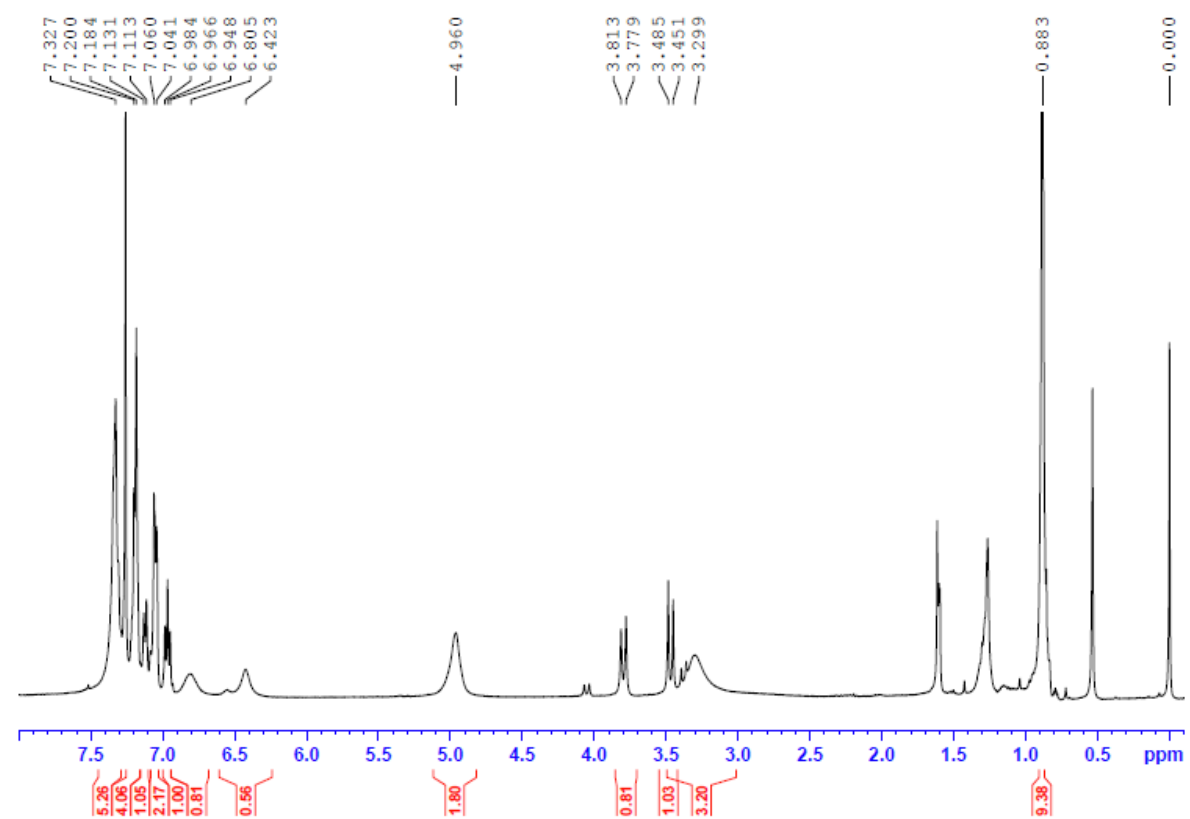

$\mathrm{CDCl}_{3},{ }^{13} \mathrm{C}\{1 \mathrm{H}\} \mathrm{NMR}, 100 \mathrm{MHz}$

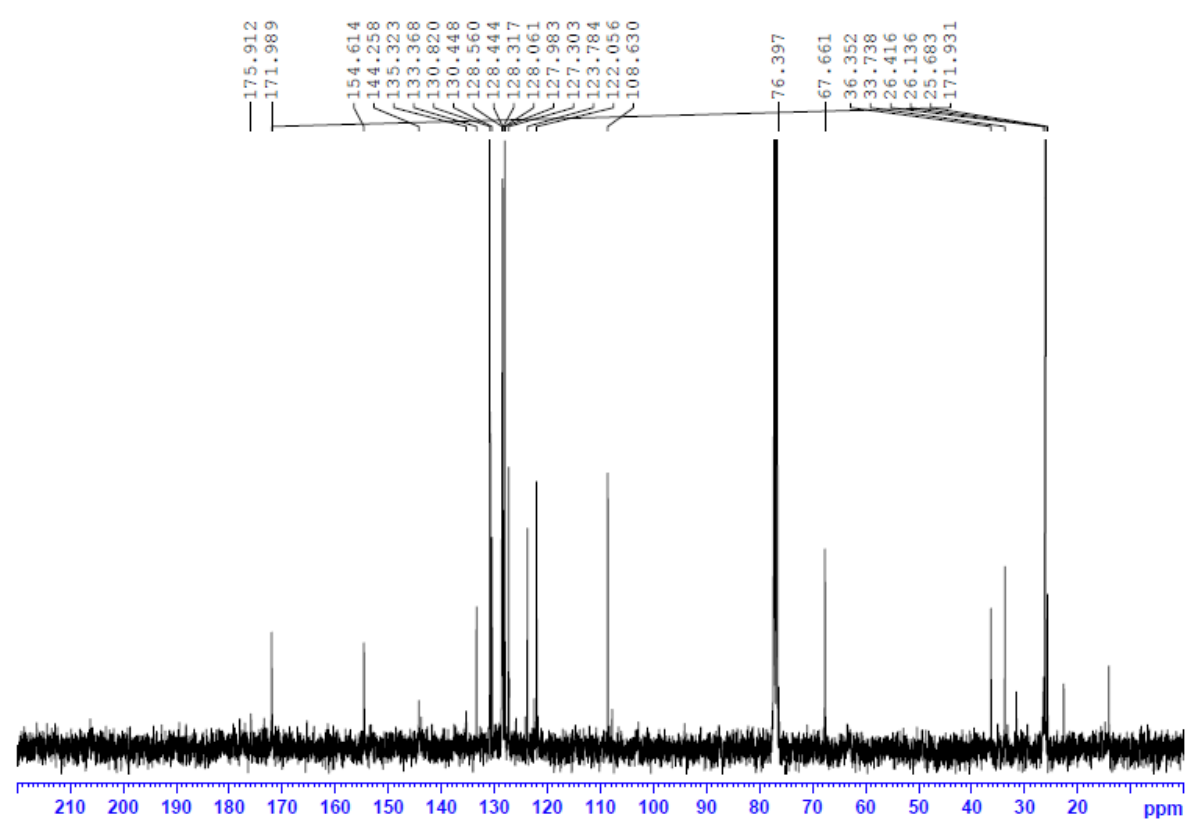




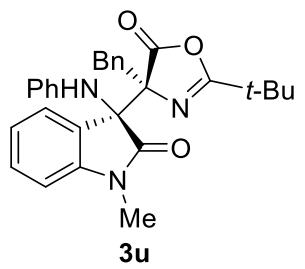

$\mathrm{CDCl}_{3},{ }^{1} \mathrm{H} \mathrm{NMR}, 400 \mathrm{MHz}$

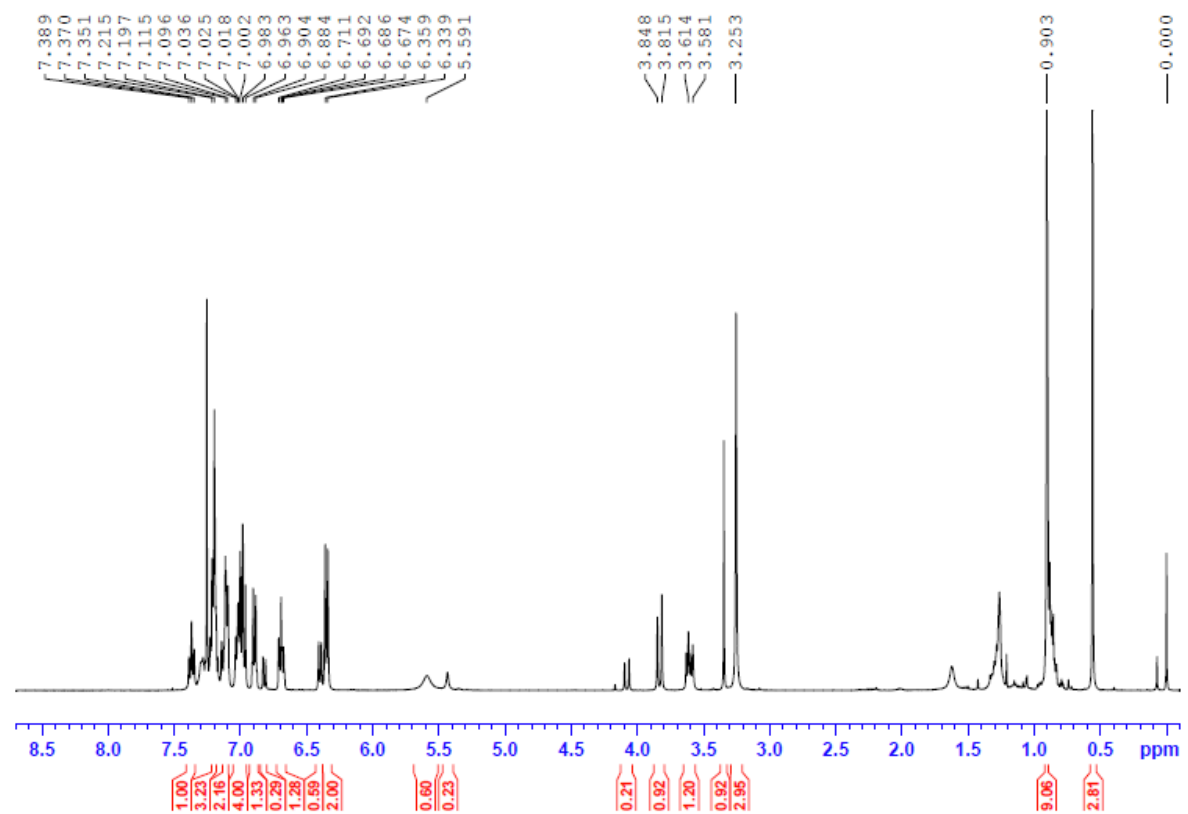

$\mathrm{CDCl}_{3},{ }^{13} \mathrm{C}\{1 \mathrm{H}\} \mathrm{NMR}, 100 \mathrm{MHz}$

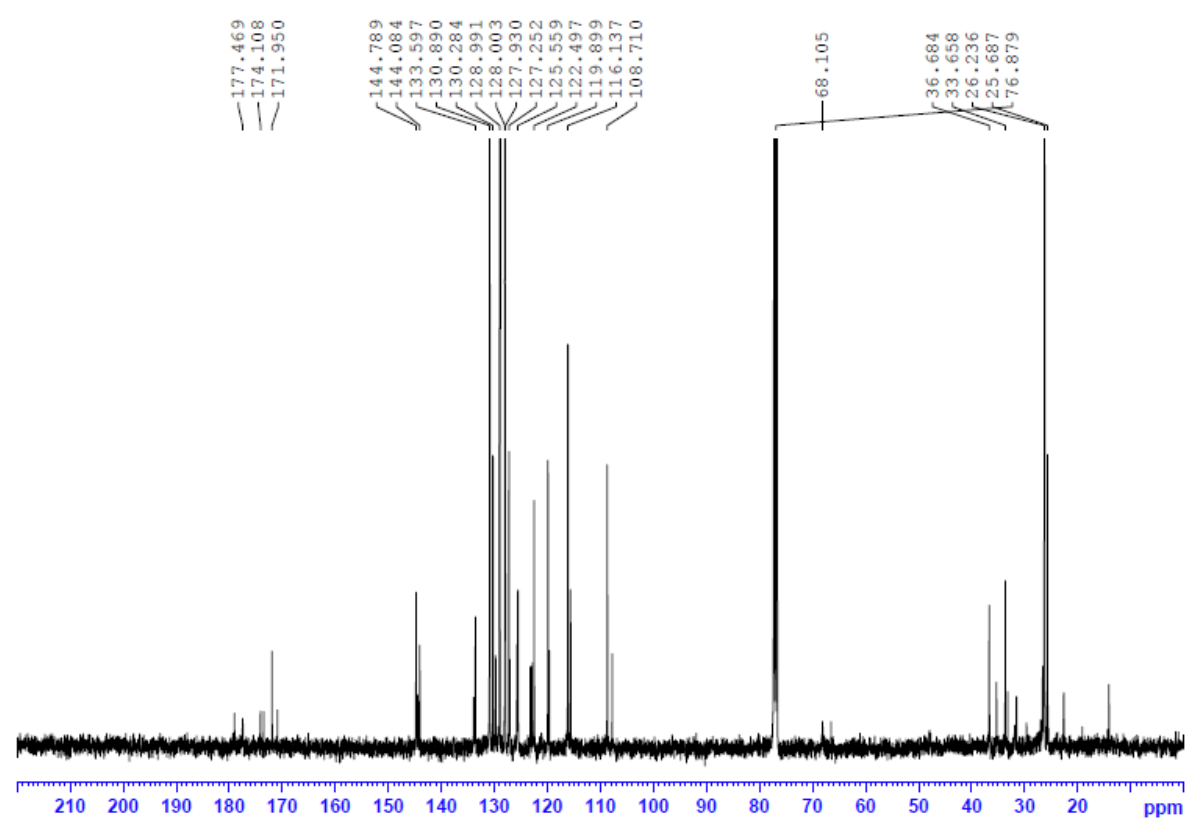




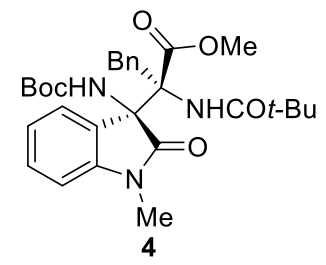

$\mathrm{CDCl}_{3},{ }^{1} \mathrm{H} \mathrm{NMR}, 400 \mathrm{MHz}$

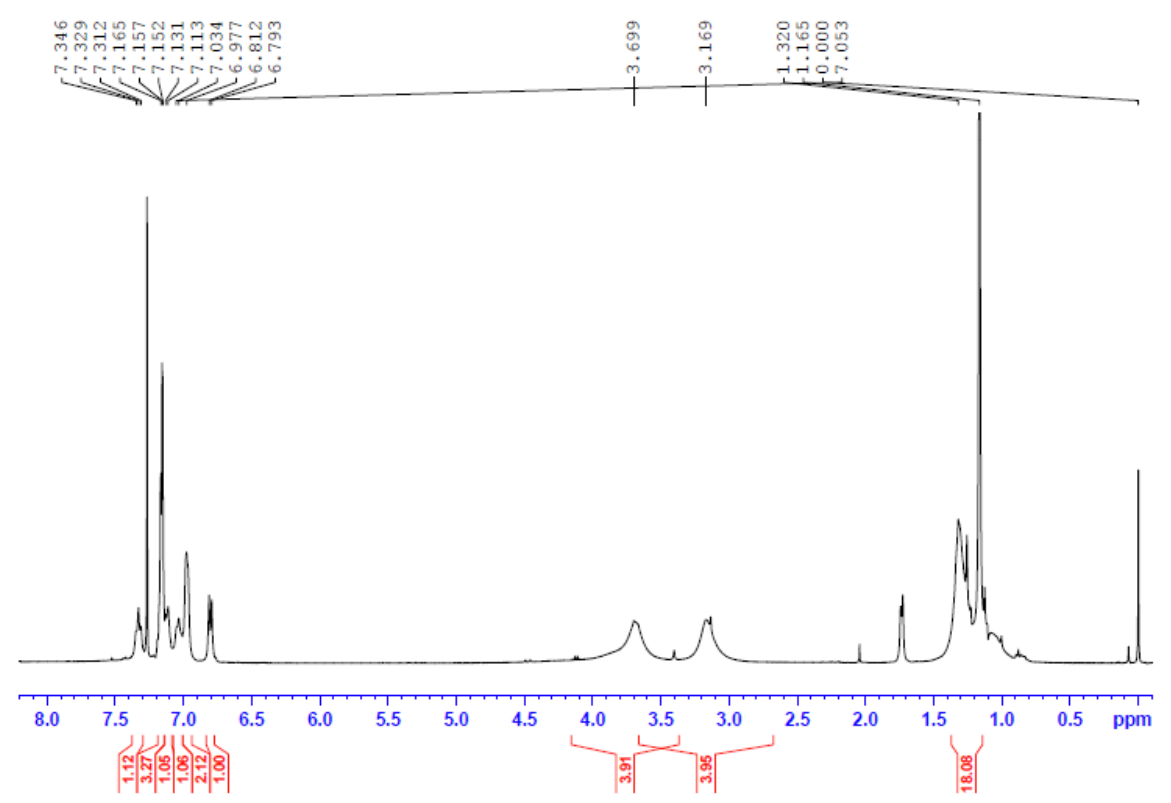

$\mathrm{CDCl}_{3},{ }^{13} \mathrm{C}\{1 \mathrm{H}\} \mathrm{NMR}, 100 \mathrm{MHz}$

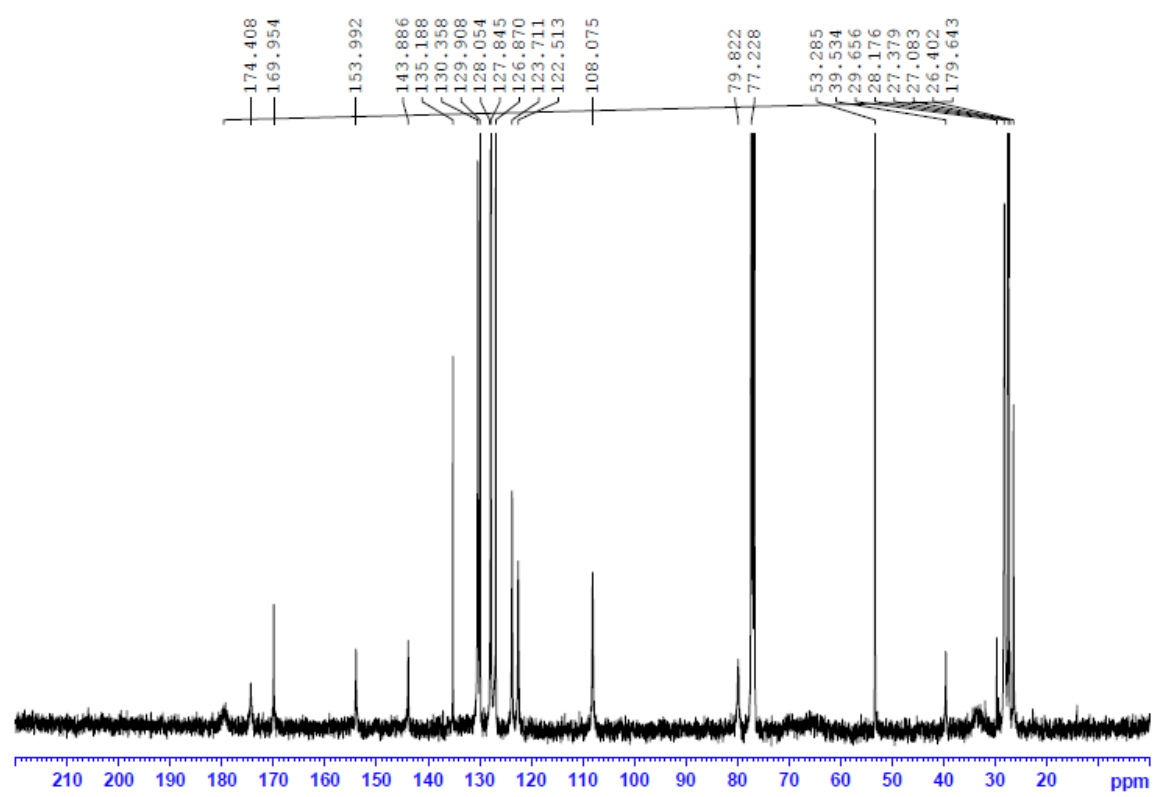



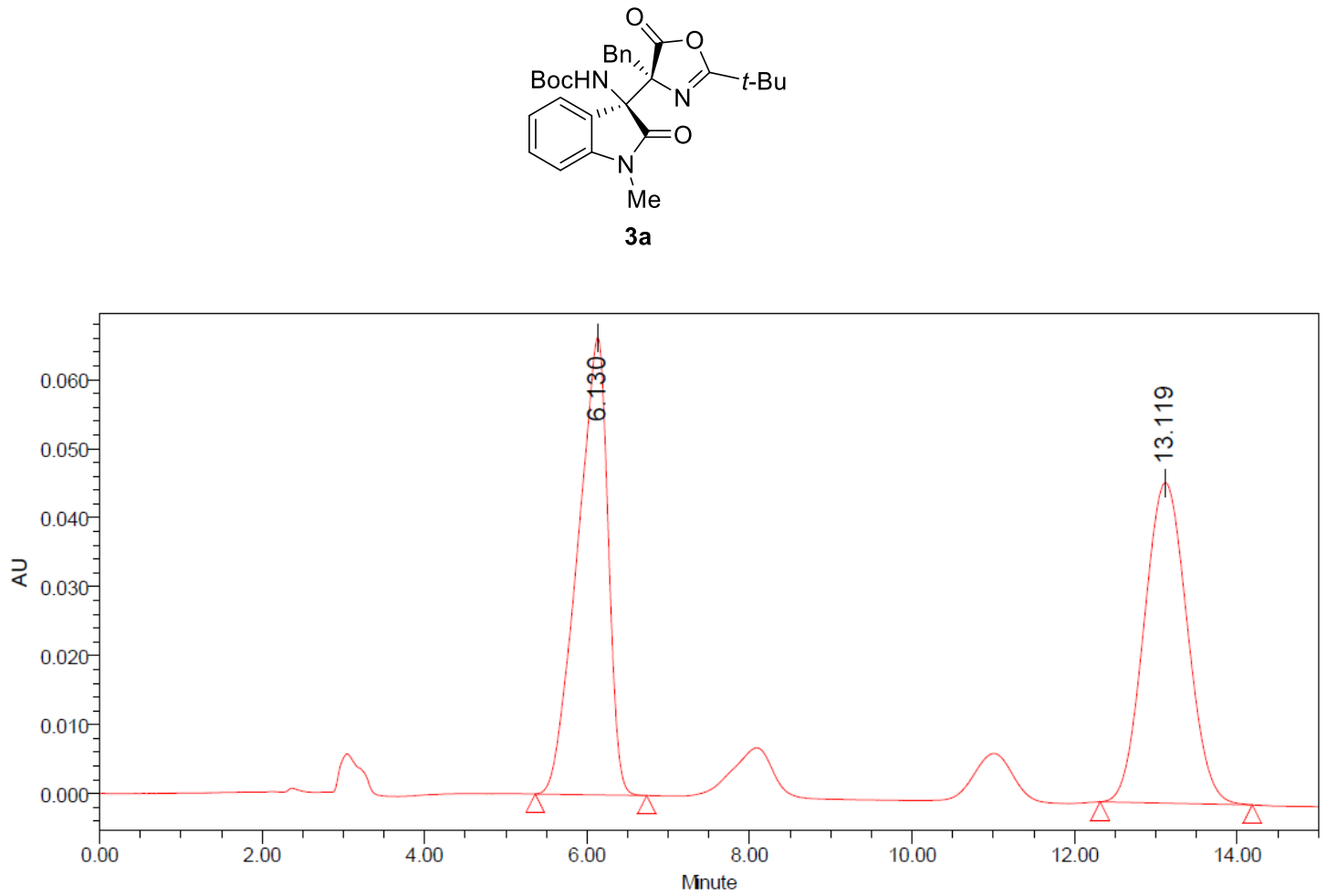

\begin{tabular}{|c|c|c|r|r|r|r|r|}
\hline & $\begin{array}{c}\text { RT } \\
(\text { Min) }\end{array}$ & $\begin{array}{c}\text { Area } \\
\left(\mu \mathrm{U}^{*} \mathrm{~s}\right)\end{array}$ & $\%$ Area & $\begin{array}{r}\text { He ight } \\
(\mu \mathrm{U})\end{array}$ & \% Height & $\begin{array}{c}\text { Start Time } \\
(\text { Min) }\end{array}$ & $\begin{array}{c}\text { End Time } \\
(\text { Min) }\end{array}$ \\
\hline 1 & 6.130 & 1726155 & 50.27 & 66252 & 58.80 & 5.362 & 6.733 \\
\hline 2 & 13.119 & 1707284 & 49.73 & 46418 & 41.20 & 12.315 & 14.188 \\
\hline
\end{tabular}

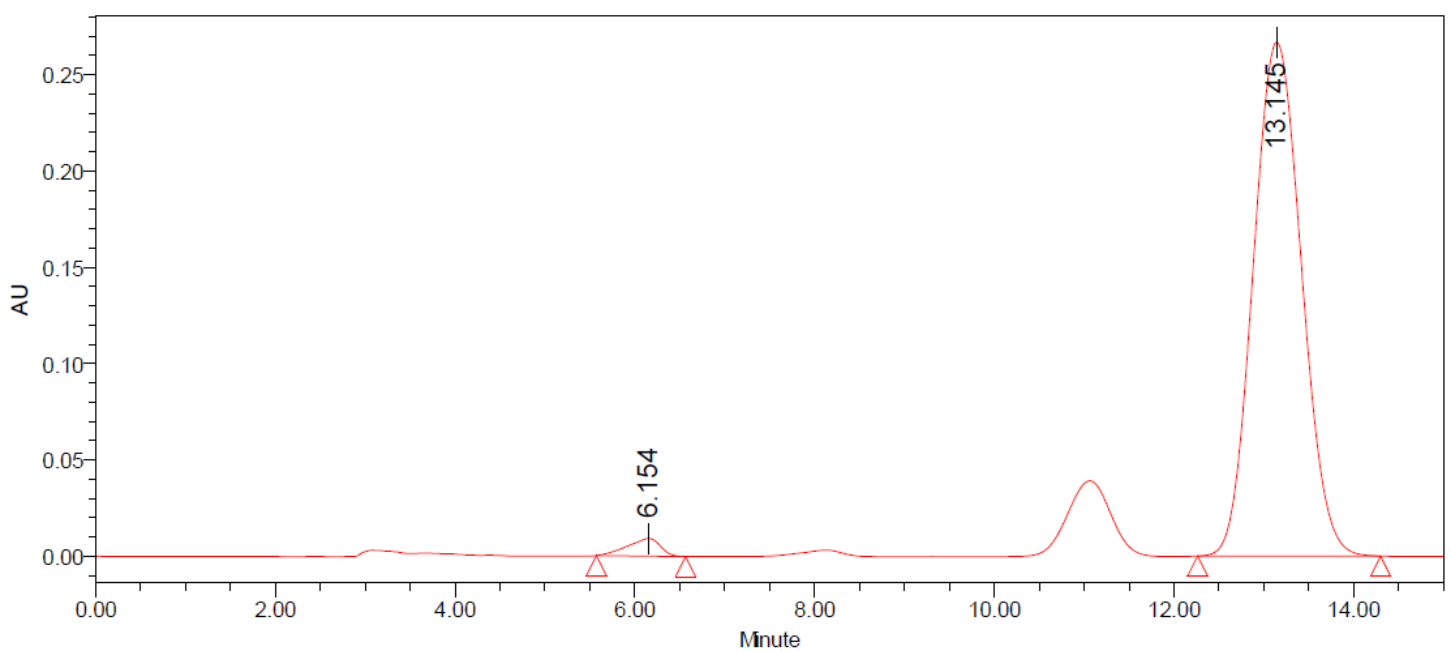

\begin{tabular}{|c|c|c|c|c|c|c|c|}
\hline & $\begin{array}{l}\text { RT } \\
\text { (Min) }\end{array}$ & $\begin{array}{c}\text { Area } \\
\left(\mu U^{*} s\right)\end{array}$ & $\%$ Area & $\begin{array}{l}\text { He ight } \\
(\mu U)\end{array}$ & $\%$ Height & $\begin{array}{l}\text { Start Time } \\
\text { (Min) }\end{array}$ & $\begin{array}{l}\text { End Time } \\
\text { (Min) }\end{array}$ \\
\hline 1 & 6.154 & 231392 & 2.27 & 9147 & 3.32 & 5.572 & 6.563 \\
\hline 2 & 13.145 & 9946293 & 97.73 & 266697 & 96.68 & 12.265 & 14.302 \\
\hline
\end{tabular}




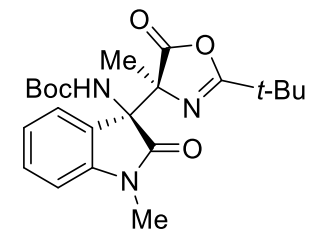

3b

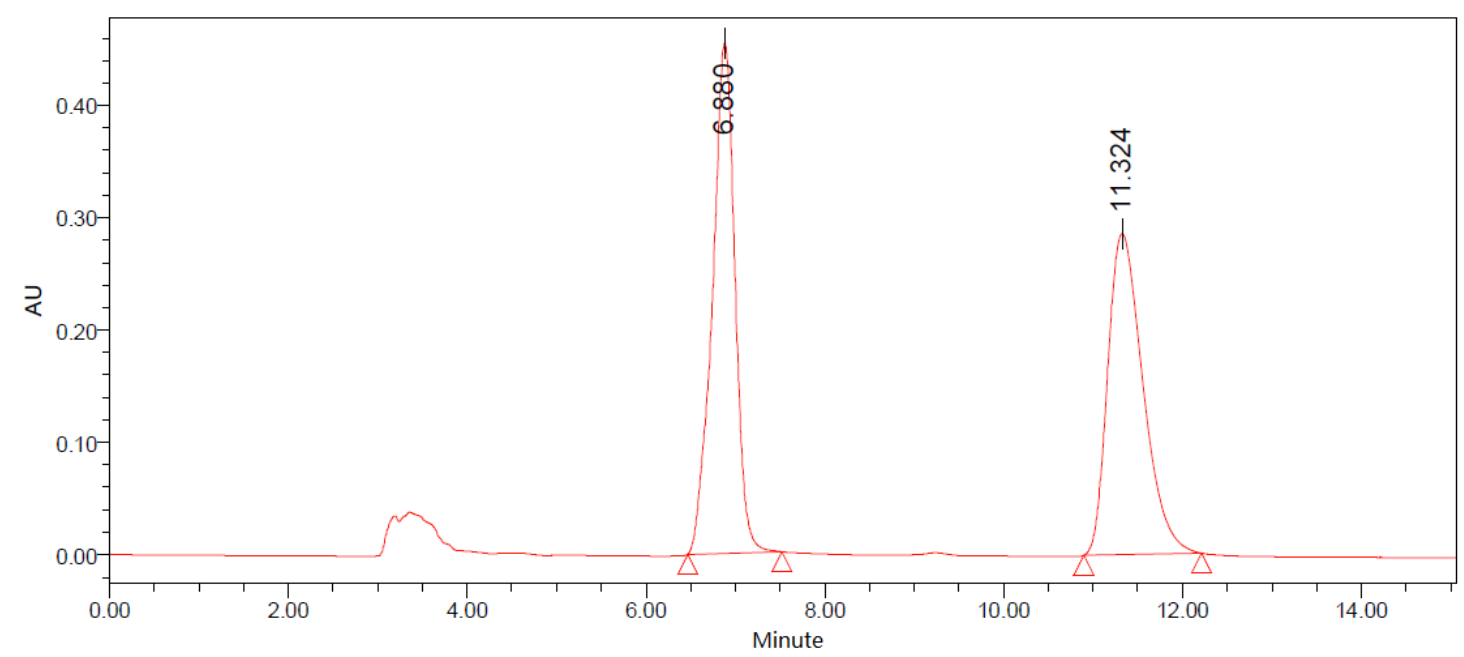

\begin{tabular}{|c|c|c|r|r|r|r|r|}
\hline & $\begin{array}{c}\text { RT } \\
(\text { Min) }\end{array}$ & $\begin{array}{c}\text { Area } \\
\left(\mu \mathrm{U}{ }^{*} \mathrm{~s}\right)\end{array}$ & $\%$ Area & $\begin{array}{c}\text { He ight } \\
(\mu \mathrm{U})\end{array}$ & $\%$ Height & $\begin{array}{c}\text { Start Time } \\
(\mathrm{Min})\end{array}$ & $\begin{array}{c}\text { End Time } \\
(\mathrm{Min})\end{array}$ \\
\hline 1 & 6.880 & 7945942 & 50.43 & 453839 & 61.38 & 6.467 & 7.517 \\
\hline 2 & 11.324 & 7810637 & 49.57 & 285593 & 38.62 & 10.897 & 12.213 \\
\hline
\end{tabular}

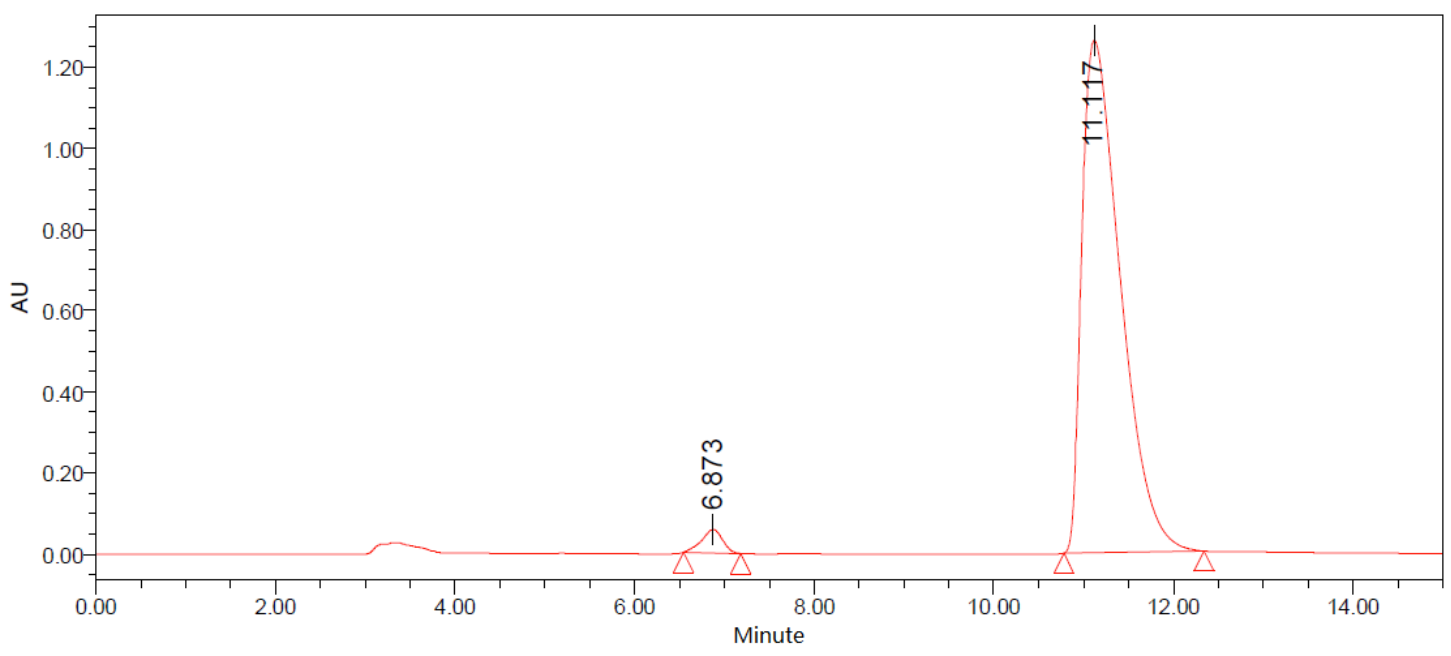

\begin{tabular}{|c|c|c|c|c|c|c|c|}
\hline & $\begin{array}{l}\text { RT } \\
\text { (Min) }\end{array}$ & $\begin{array}{c}\text { Area } \\
\left(u U^{*} s\right)\end{array}$ & $\%$ Area & $\begin{array}{l}\text { He ight } \\
(\mu U)\end{array}$ & $\%$ Height & $\underset{\text { (Min) }}{\text { Start Time }}$ & $\begin{array}{l}\text { End Time } \\
\text { (Min) }\end{array}$ \\
\hline 1 & 6.873 & 940003 & 2.43 & 57863 & 4.38 & 6.545 & 7.182 \\
\hline 2 & 11.117 & 37746334 & 97.57 & 1262888 & 95.62 & 10.778 & 12.335 \\
\hline
\end{tabular}



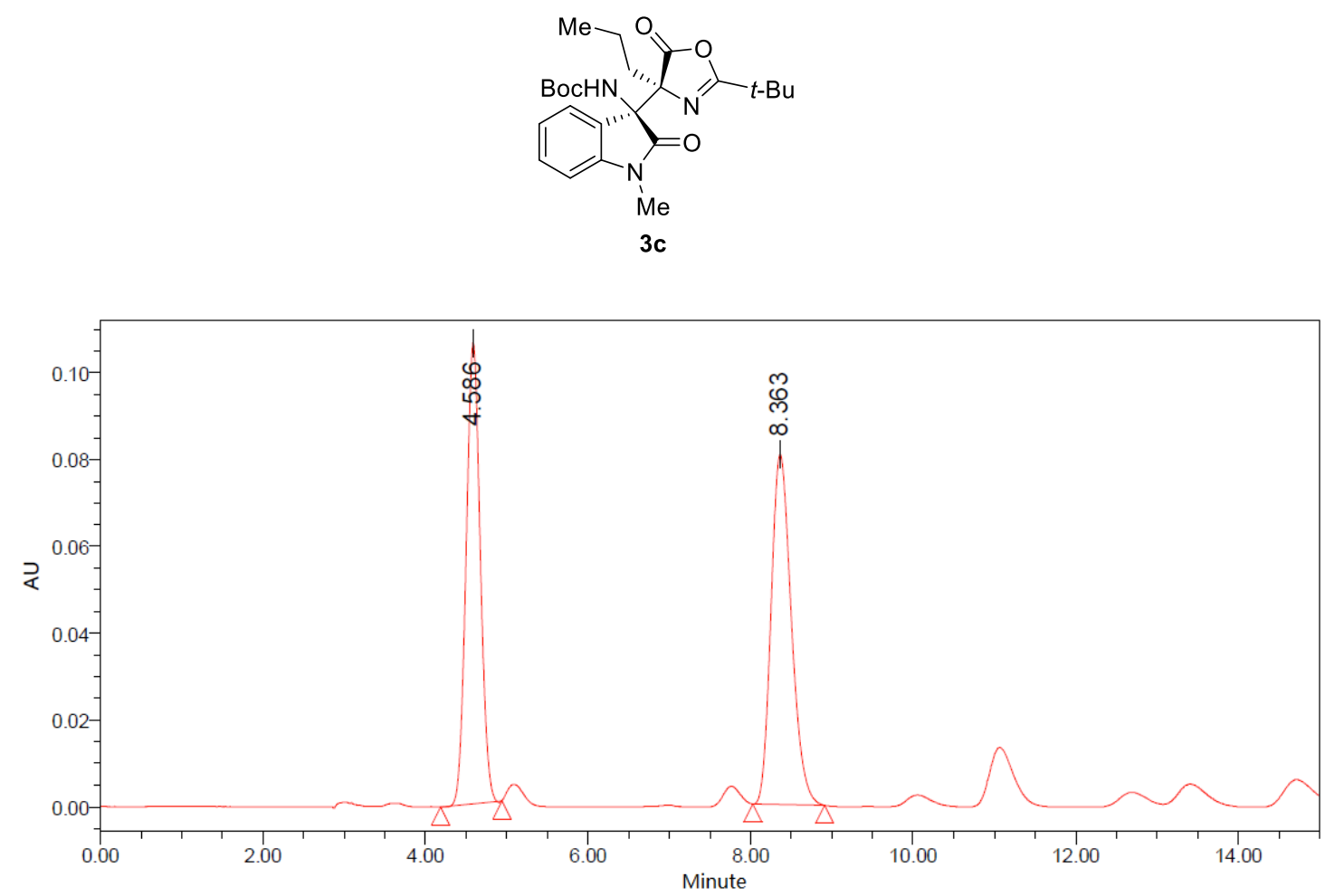

\begin{tabular}{|l|c|c|r|r|r|r|r|}
\hline & $\begin{array}{c}\text { RT } \\
(\text { Min) }\end{array}$ & $\begin{array}{c}\text { Area } \\
\left(\mu \mathrm{U}^{*} \mathrm{~s}\right)\end{array}$ & $\%$ Area & $\begin{array}{c}\text { He ight } \\
(\mu \mathrm{U})\end{array}$ & $\%$ Height & $\begin{array}{c}\text { Start Time } \\
(\mathrm{Min})\end{array}$ & $\begin{array}{c}\text { End Time } \\
(\mathrm{Min})\end{array}$ \\
\hline 1 & 4.586 & 1281231 & 47.82 & 106131 & 56.80 & 4.183 & 4.938 \\
\hline 2 & 8.363 & 1397899 & 52.18 & 80713 & 43.20 & 8.030 & 8.912 \\
\hline
\end{tabular}

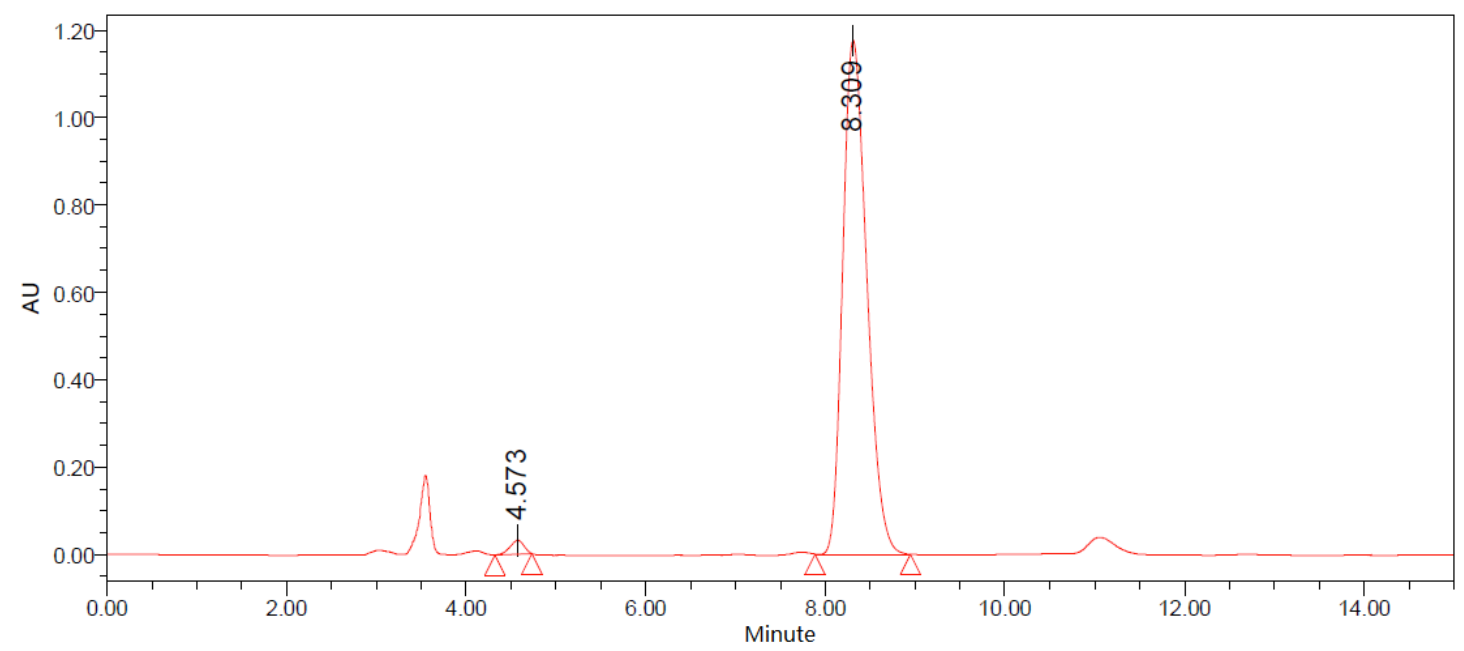

\begin{tabular}{|l|c|r|r|r|r|r|r|}
\hline & $\begin{array}{c}\mathrm{RT} \\
(\mathrm{Min})\end{array}$ & \multicolumn{1}{|c|}{$\begin{array}{c}\text { Area } \\
\left(\mu \mathrm{U}^{*} \mathrm{~s}\right)\end{array}$} & $\%$ Area & $\begin{array}{c}\text { He ight } \\
(\mu \mathrm{U})\end{array}$ & $\%$ He ight & $\begin{array}{c}\text { Start Time } \\
(\text { Min })\end{array}$ & $\begin{array}{c}\text { End Time } \\
(\mathrm{Min})\end{array}$ \\
\hline 1 & 4.573 & 347374 & 1.56 & 32153 & 2.66 & 4.318 & 4.735 \\
\hline 2 & 8.309 & 21928514 & 98.44 & 1176923 & 97.34 & 7.885 & 8.945 \\
\hline
\end{tabular}



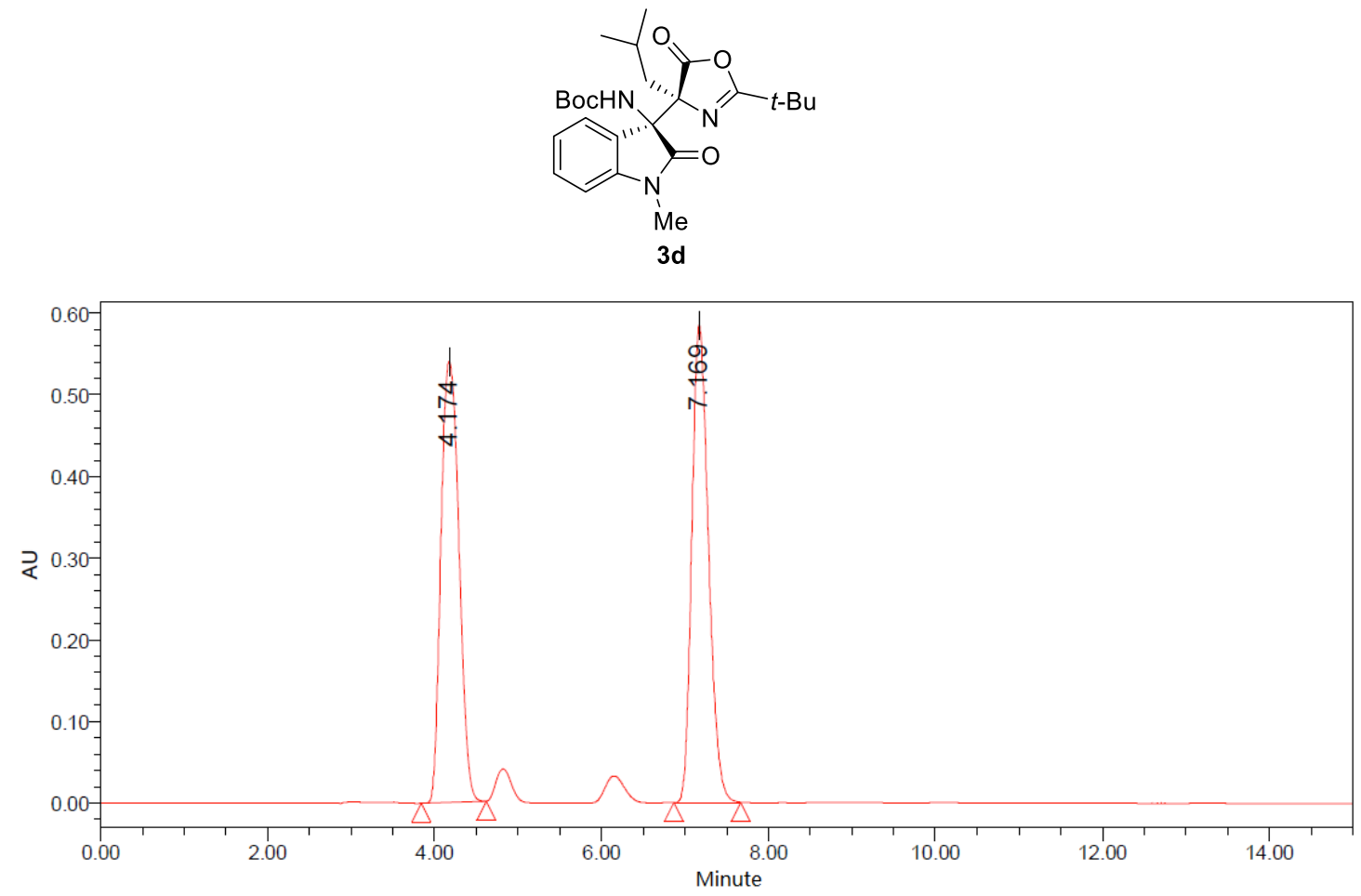

\begin{tabular}{|l|c|c|r|r|r|r|r|}
\hline & $\begin{array}{c}\text { RT } \\
(\text { Min) }\end{array}$ & $\begin{array}{c}\text { Area } \\
\left(\mu U^{*} \mathrm{~s}\right)\end{array}$ & $\%$ Area & $\begin{array}{c}\text { He ight } \\
(\mu \mathrm{U})\end{array}$ & $\%$ Height & $\begin{array}{c}\text { Start Time } \\
(\text { Min) }\end{array}$ & $\begin{array}{l}\text { End Time } \\
(\text { Min) }\end{array}$ \\
\hline 1 & 4.174 & 8101232 & 49.97 & 540044 & 48.03 & 3.843 & 4.622 \\
\hline 2 & 7.169 & 8111047 & 50.03 & 584257 & 51.97 & 6.868 & 7.670 \\
\hline
\end{tabular}

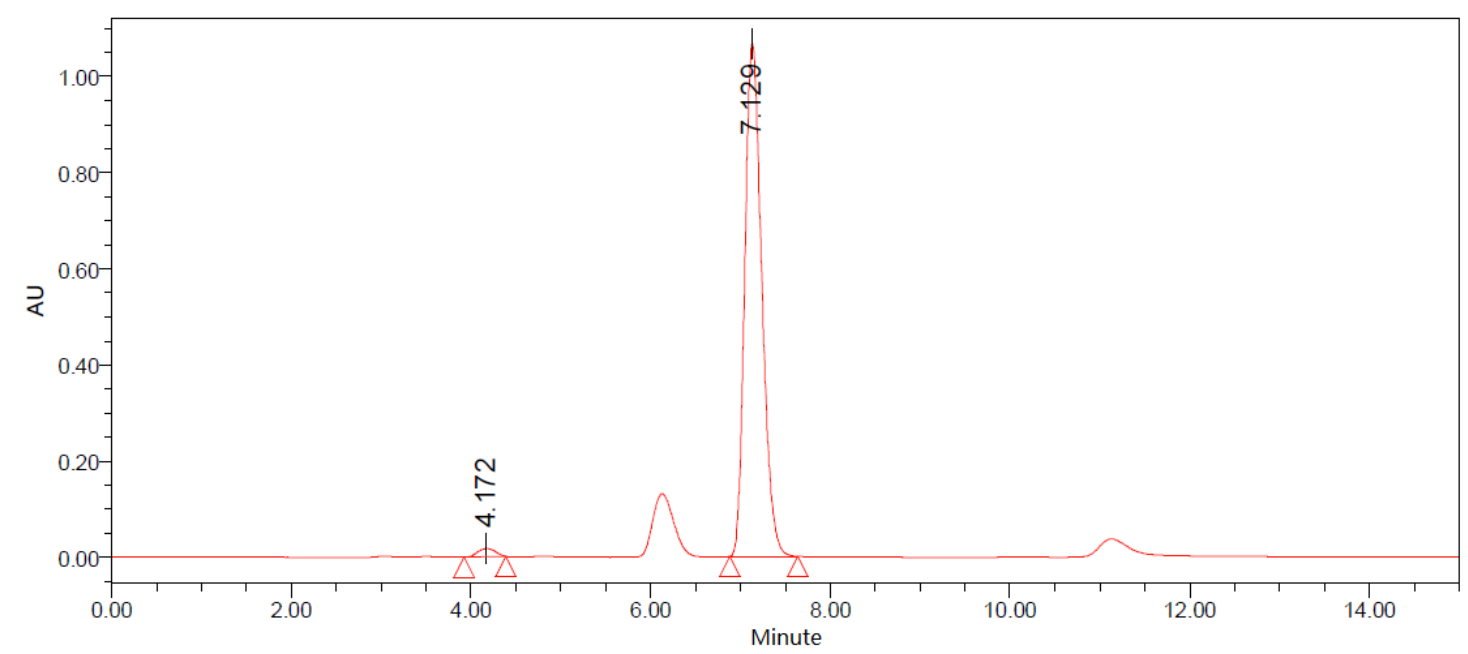

\begin{tabular}{|c|c|c|c|c|c|c|c|}
\hline & $\begin{array}{l}\text { RT } \\
\text { (Min) }\end{array}$ & $\begin{array}{c}\text { Area } \\
\left(\mu U^{*} s\right)\end{array}$ & $\%$ Area & $\begin{array}{l}\text { He ight } \\
(\mu U)\end{array}$ & $\%$ Height & $\begin{array}{l}\text { Start Time } \\
\text { (Min) }\end{array}$ & $\begin{array}{l}\text { End Time } \\
\text { (Min) }\end{array}$ \\
\hline 1 & 4.172 & 235711 & 1.66 & 17223 & 1.59 & 3.923 & 4.385 \\
\hline 2 & 7.129 & 13992497 & 98.34 & 1066827 & 98.41 & 6.880 & 7.637 \\
\hline
\end{tabular}



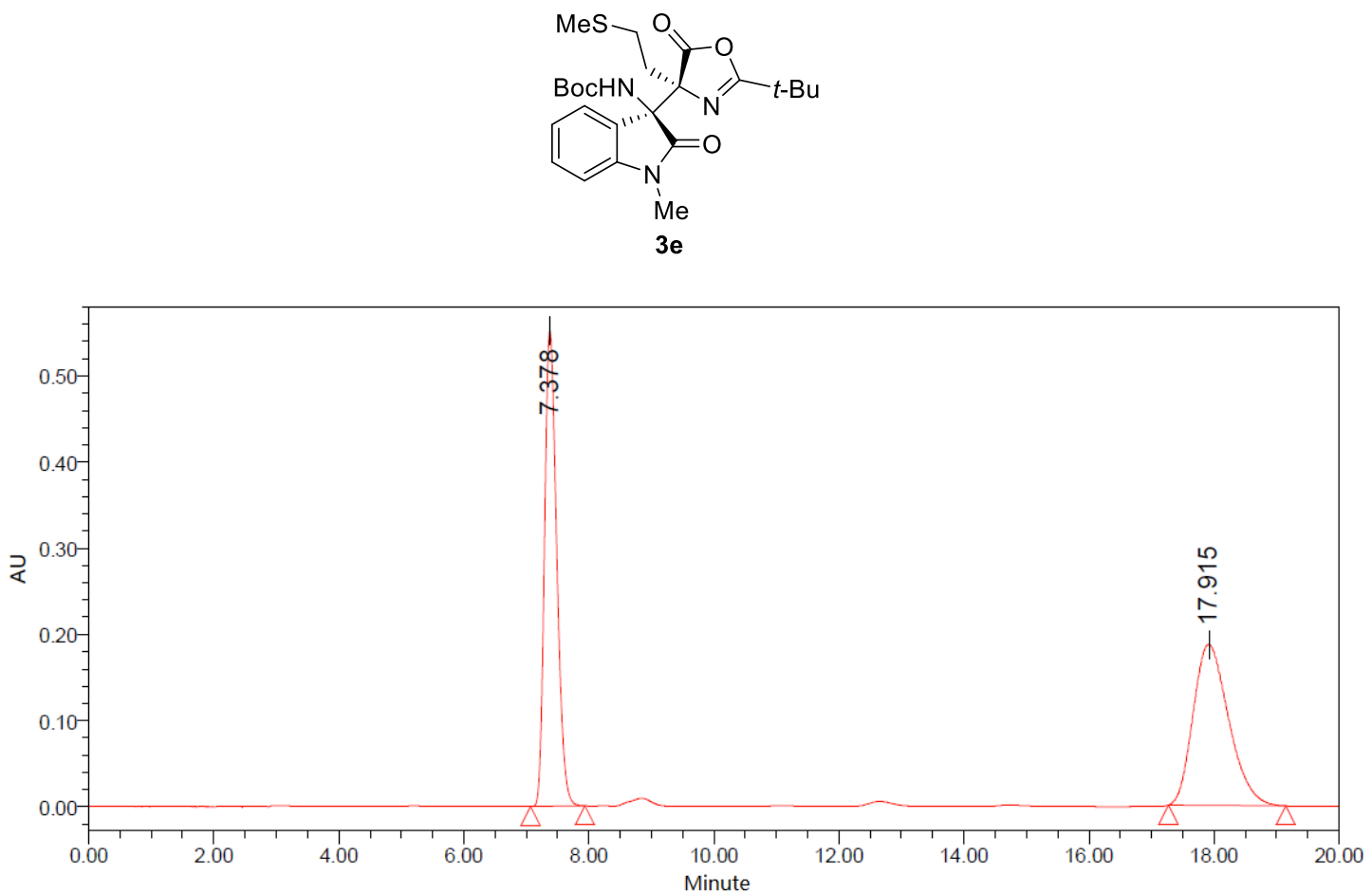

\begin{tabular}{|l|r|r|r|r|r|r|r|}
\hline & $\begin{array}{c}\text { RT } \\
(\text { Min) }\end{array}$ & $\begin{array}{c}\text { Area } \\
\left(\mu U^{*} \mathrm{~s}\right)\end{array}$ & $\%$ Area & $\begin{array}{c}\text { He ight } \\
(\mu \mathrm{U})\end{array}$ & $\%$ Height & $\begin{array}{c}\text { Start Time } \\
(\text { Min) }\end{array}$ & $\begin{array}{r}\text { End Time } \\
(\text { Min) }\end{array}$ \\
\hline 1 & 7.378 & 7489354 & 50.37 & 552691 & 74.71 & 7.067 & 7.938 \\
\hline 2 & 17.915 & 7377992 & 49.63 & 187100 & 25.29 & 17.268 & 19.150 \\
\hline
\end{tabular}

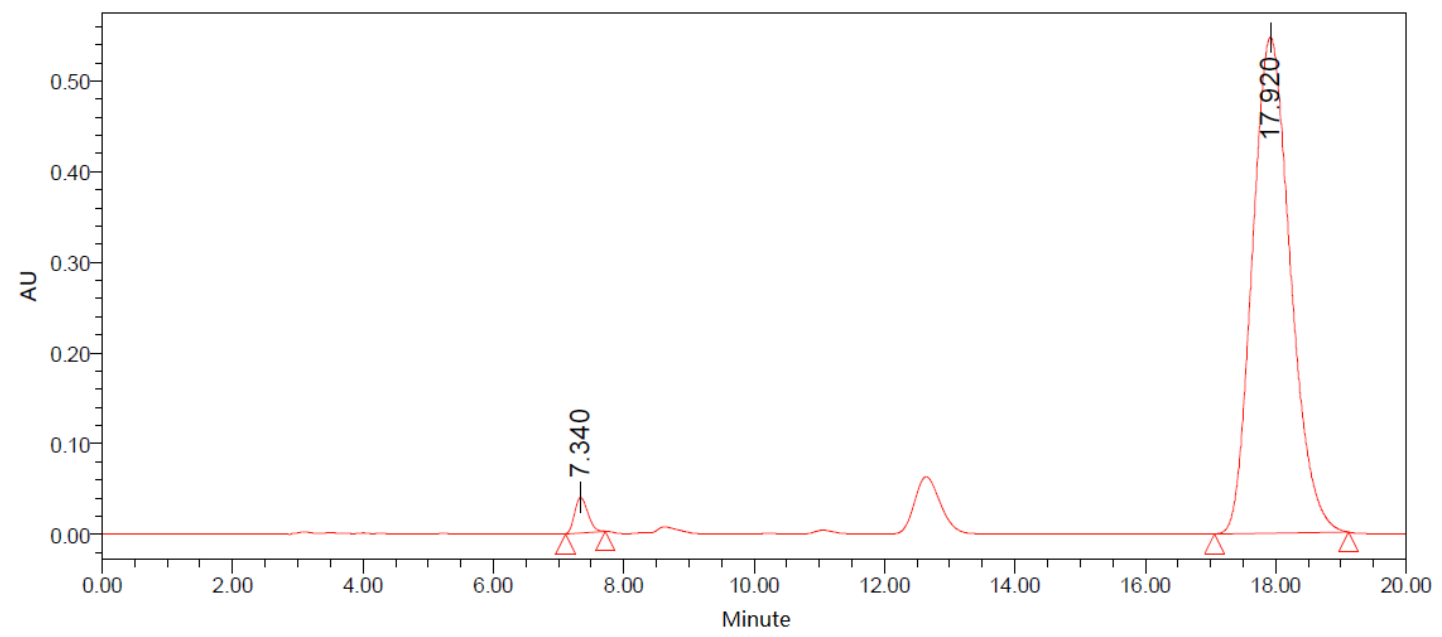

\begin{tabular}{|l|r|r|r|r|r|r|r|}
\hline & $\begin{array}{c}\text { RT } \\
(\text { Min) }\end{array}$ & $\begin{array}{c}\text { Area } \\
\left(\mu \mathrm{U}^{*} \mathrm{~s}\right)\end{array}$ & \% Area & $\begin{array}{c}\text { He ight } \\
(\mu \mathrm{U})\end{array}$ & $\%$ Height & $\begin{array}{c}\text { Start Time } \\
(\text { Min) }\end{array}$ & $\begin{array}{c}\text { End Time } \\
(\text { Min) }\end{array}$ \\
\hline 1 & 7.340 & 545497 & 2.41 & 39032 & 6.66 & 7.110 & 7.713 \\
\hline 2 & 17.920 & 22081385 & 97.59 & 547197 & 93.34 & 17.058 & 19.120 \\
\hline
\end{tabular}



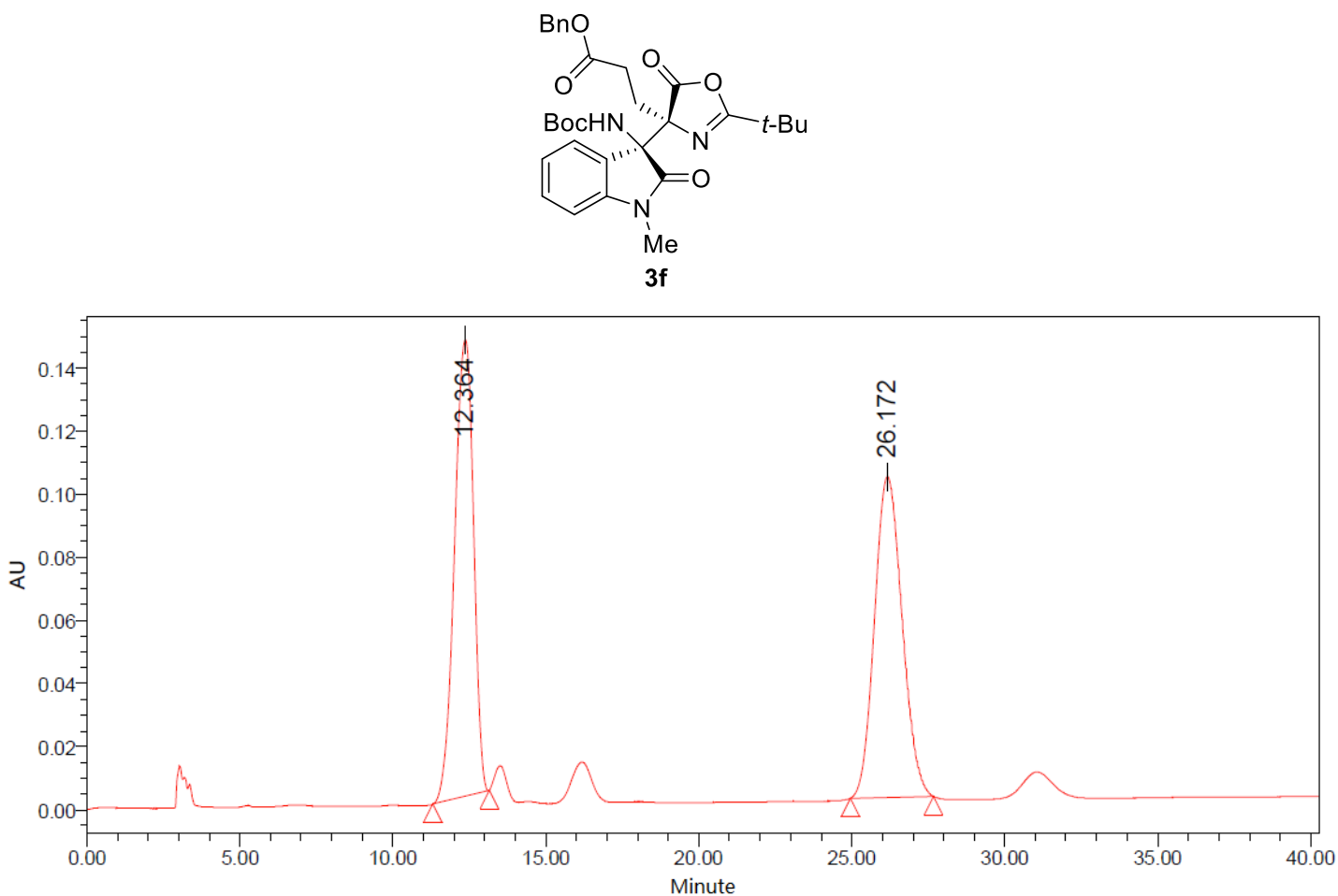

\begin{tabular}{|l|c|c|r|r|r|r|r|}
\hline & $\begin{array}{c}\text { RT } \\
(\mathrm{Min})\end{array}$ & $\begin{array}{c}\text { Area } \\
\left(\mu \mathrm{U}^{*} \mathrm{~s}\right)\end{array}$ & $\%$ Area & $\begin{array}{c}\text { He ight } \\
(\mu \mathrm{U})\end{array}$ & $\%$ Height & $\begin{array}{c}\text { Start Time } \\
(\mathrm{Min})\end{array}$ & $\begin{array}{c}\text { End Time } \\
(\mathrm{Min})\end{array}$ \\
\hline 1 & 12.364 & 6235866 & 49.52 & 144579 & 58.74 & 11.300 & 13.153 \\
\hline 2 & 26.172 & 6356720 & 50.48 & 101571 & 41.26 & 24.950 & 27.663 \\
\hline
\end{tabular}

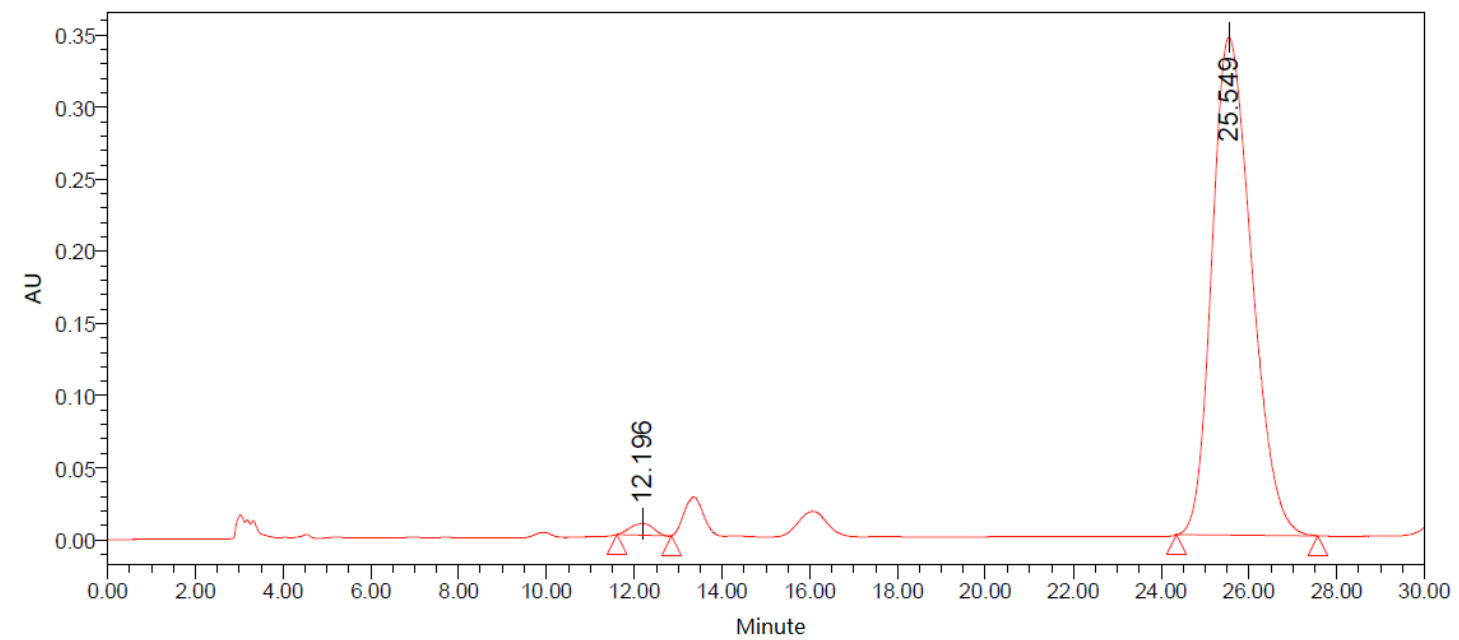

\begin{tabular}{|r|r|r|r|r|r|r|r|}
\hline & $\begin{array}{c}\text { RT } \\
(\text { Min })\end{array}$ & \multicolumn{1}{c|}{$\begin{array}{c}\text { Area } \\
\left(\mu \mathrm{U}^{*}\right)\end{array}$} & \% Area & $\begin{array}{c}\text { He ight } \\
(\mu \mathrm{U})\end{array}$ & $\%$ Height & $\begin{array}{c}\text { Start Time } \\
(\text { Min })\end{array}$ & $\begin{array}{c}\text { End Time } \\
(\text { Min })\end{array}$ \\
\hline 1 & 12.196 & 296382 & 1.35 & 8022 & 2.27 & 11.602 & 12.843 \\
\hline 2 & 25.549 & 21685984 & 98.65 & 345523 & 97.73 & 24.352 & 27.573 \\
\hline
\end{tabular}



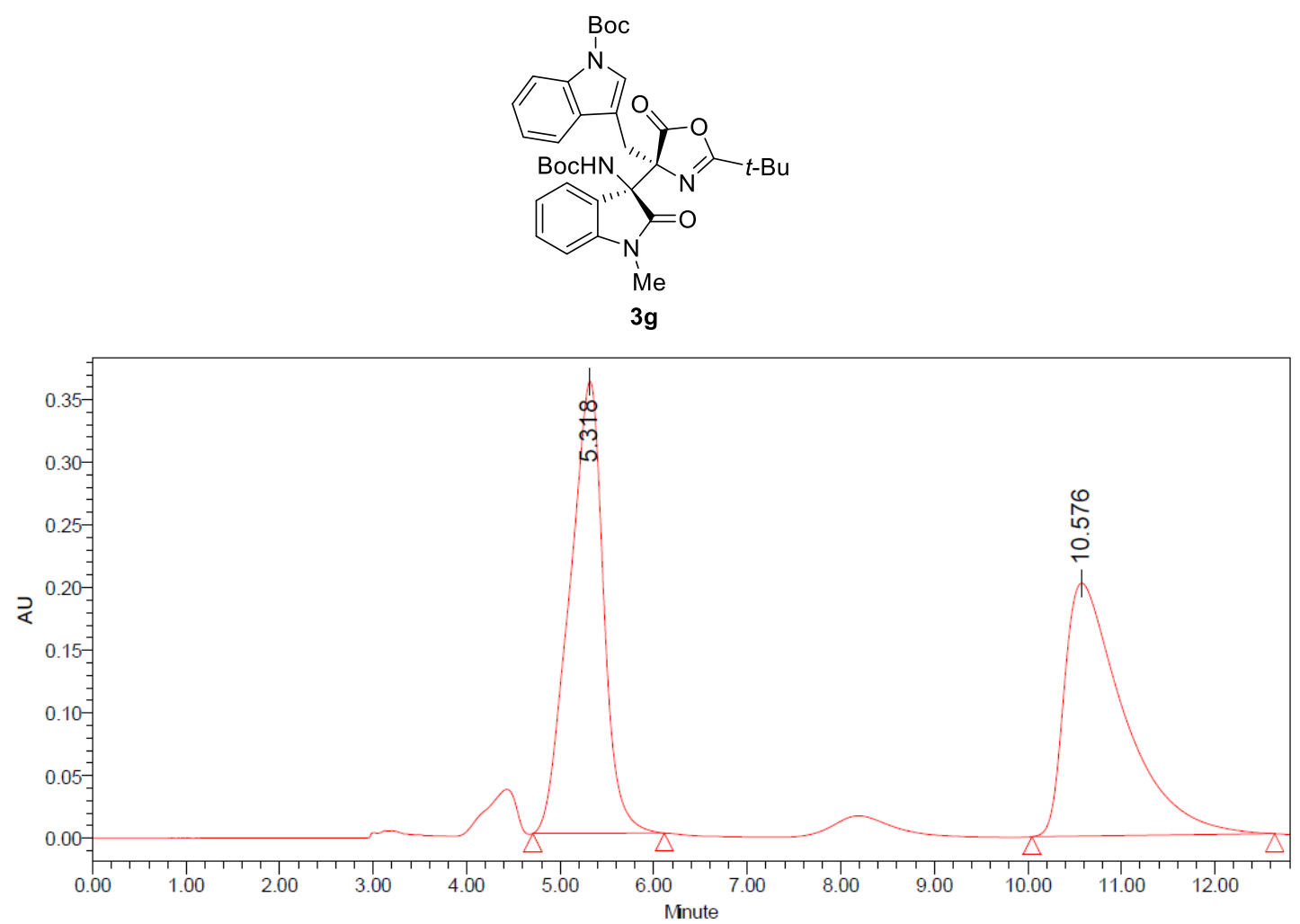

\begin{tabular}{|c|c|c|c|c|c|c|c|}
\hline & $\begin{array}{l}\mathbf{R T} \\
\text { (Min) }\end{array}$ & $\begin{array}{c}\text { Area } \\
\left(\mu U^{*} s\right)\end{array}$ & $\%$ Area & $\begin{array}{l}\text { He ight } \\
(\mu \mathrm{U})\end{array}$ & $\%$ Height & $\begin{array}{l}\text { Start Time } \\
\text { (Min) }\end{array}$ & $\begin{array}{l}\text { End Time } \\
\text { (Min) }\end{array}$ \\
\hline 1 & 5.318 & 9104047 & 50.47 & 360695 & 64.12 & 4.707 & 6.113 \\
\hline 2 & 10.576 & 8934269 & 49.53 & 201807 & 35.88 & 10.043 & 12.640 \\
\hline
\end{tabular}

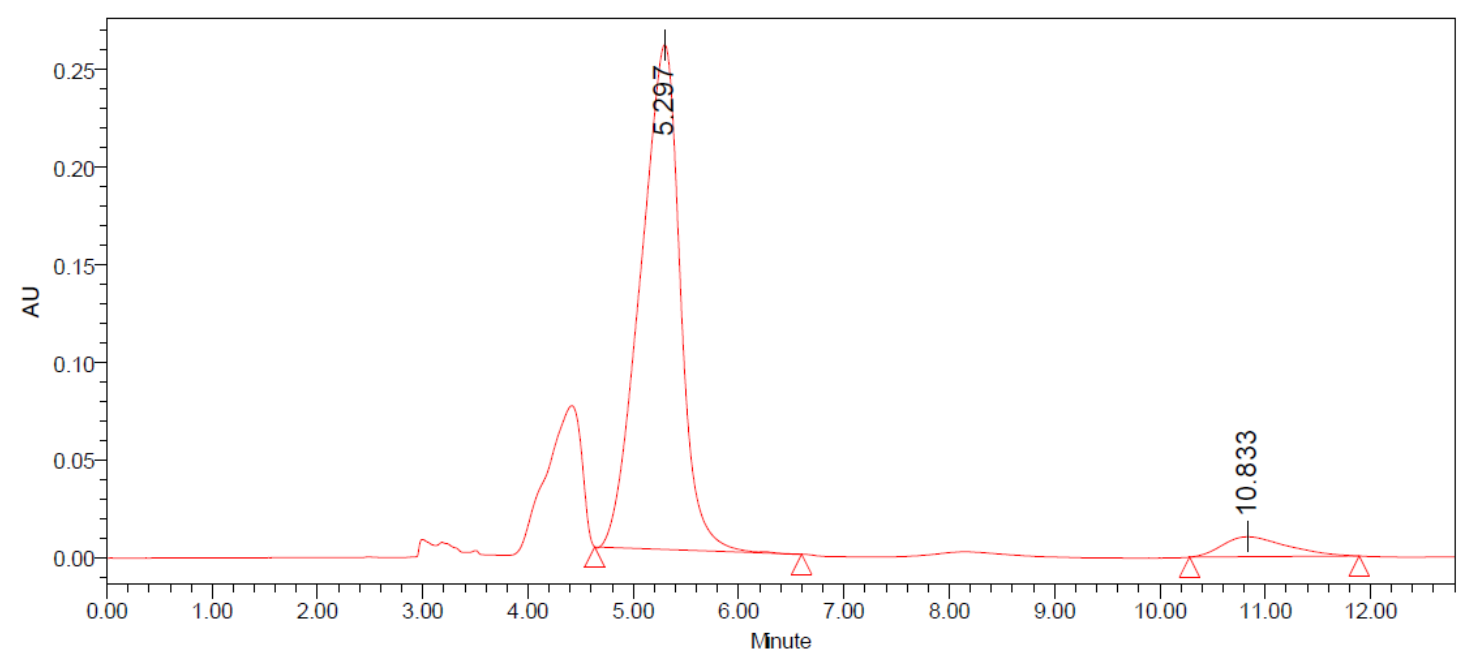

\begin{tabular}{|c|c|c|c|c|c|c|c|}
\hline & $\begin{array}{l}\mathbf{R T} \\
\text { (Min) }\end{array}$ & $\begin{array}{l}\text { Area } \\
\left(\mu U^{*} \mathrm{~s}\right)\end{array}$ & $\%$ Area & $\begin{array}{l}\text { He ight } \\
(\mu U)\end{array}$ & $\%$ Height & $\underset{\text { (Min) }}{\text { Start Time }}$ & $\begin{array}{l}\text { End Time } \\
\text { (Min) }\end{array}$ \\
\hline 1 & 5.297 & 6783256 & 93.95 & 258286 & 96.18 & 4.632 & 6.597 \\
\hline 2 & 10.833 & 437093 & 6.05 & 10246 & 3.82 & 10.282 & 11.893 \\
\hline
\end{tabular}



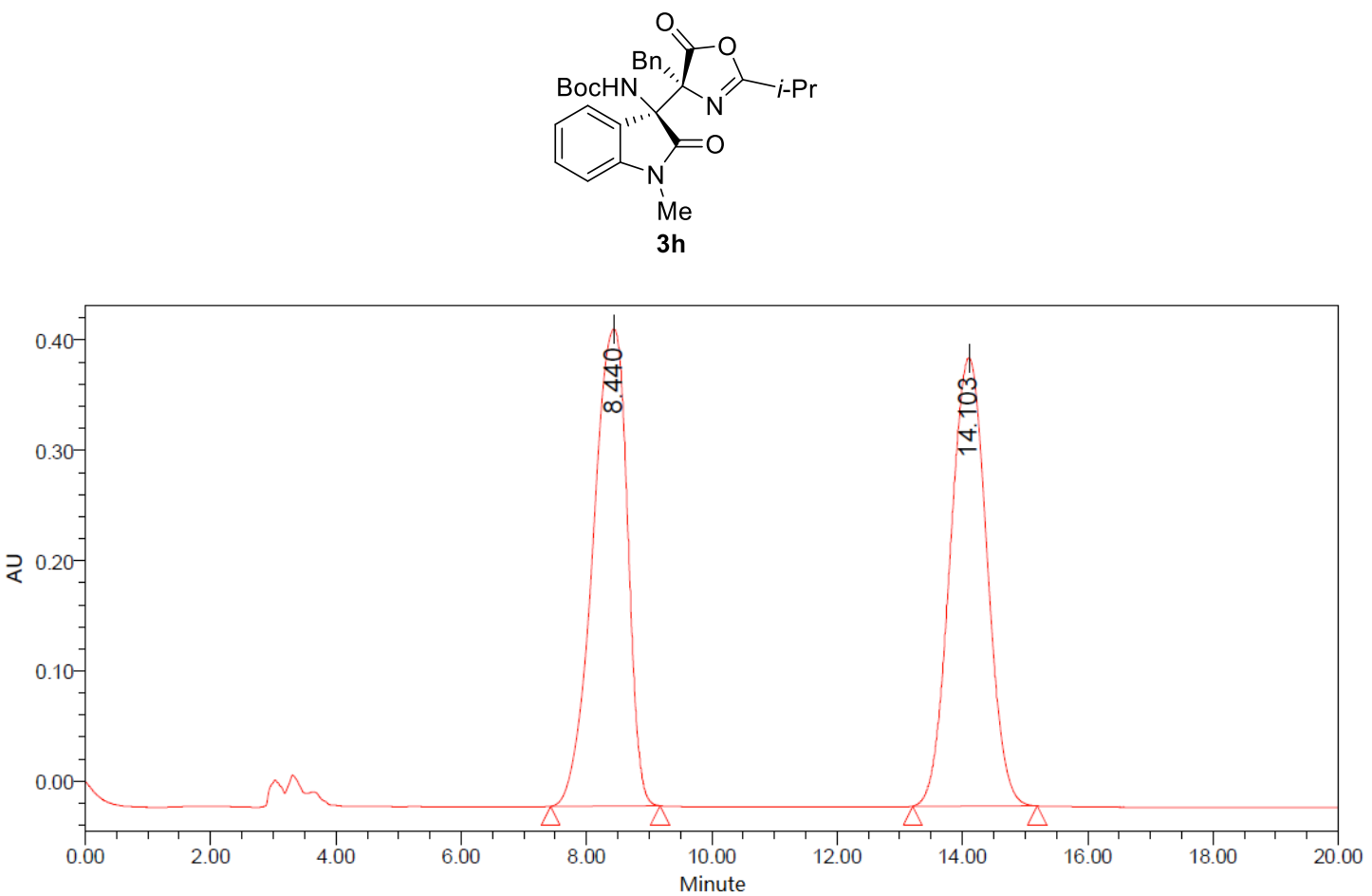

\begin{tabular}{|c|c|c|r|r|r|r|r|}
\hline & $\begin{array}{c}\mathbf{R T} \\
(\mathrm{Min})\end{array}$ & $\begin{array}{c}\text { Area } \\
\left(\mu \mathrm{U}^{*} \mathrm{~s}\right)\end{array}$ & \% Area & $\begin{array}{c}\text { He ight } \\
(\mu \mathrm{U})\end{array}$ & $\%$ Height & $\begin{array}{c}\text { Start Time } \\
(\text { Min) }\end{array}$ & $\begin{array}{c}\text { End Time } \\
(\mathrm{Min})\end{array}$ \\
\hline 1 & 8.440 & 16537445 & 50.02 & 432522 & 51.56 & 7.428 & 9.175 \\
\hline 2 & 14.103 & 16521488 & 49.98 & 406323 & 48.44 & 13.212 & 15.197 \\
\hline
\end{tabular}

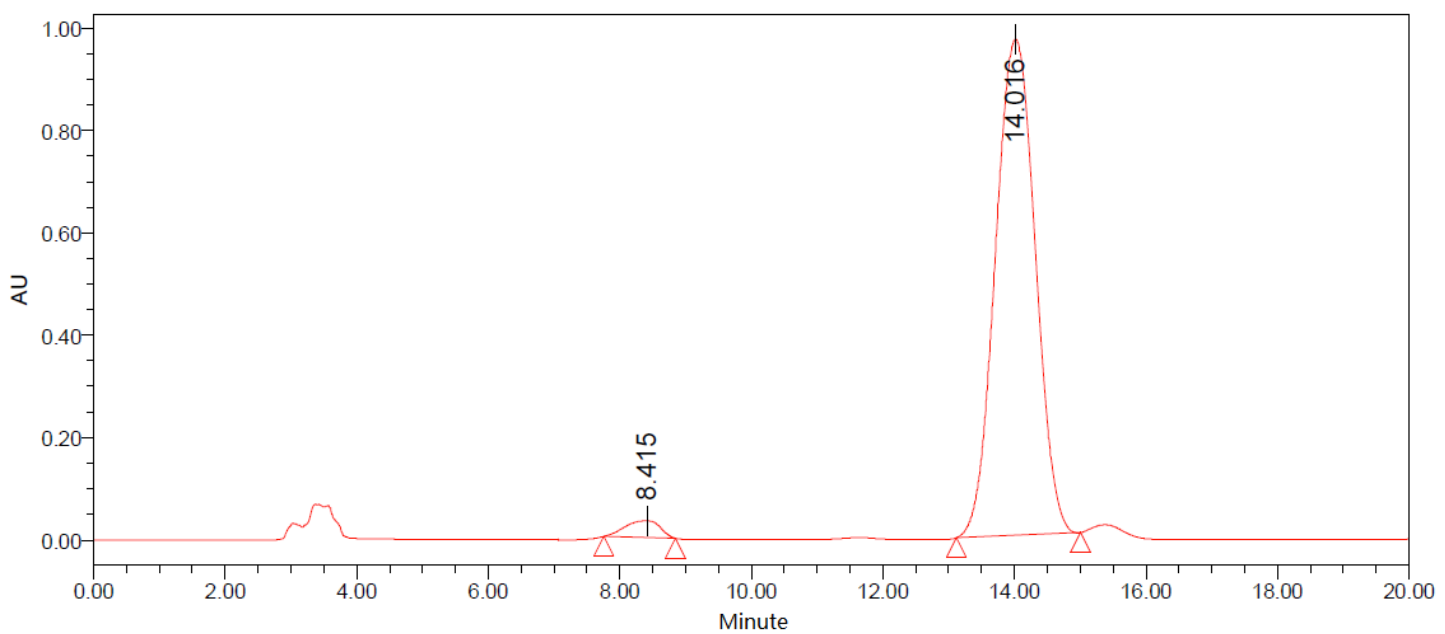

\begin{tabular}{|c|c|c|c|c|c|c|c|}
\hline & $\begin{array}{l}\mathbf{R T} \\
\text { (Min) }\end{array}$ & $\begin{array}{l}\text { Area } \\
\left(\mu U^{*} s\right)\end{array}$ & $\%$ Area & $\begin{array}{l}\text { He ight } \\
(\mu U)\end{array}$ & $\%$ Height & $\begin{array}{l}\text { Start Time } \\
\text { (Min) }\end{array}$ & $\begin{array}{l}\text { End Time } \\
\text { (Min) }\end{array}$ \\
\hline 1 & 8.415 & 1210482 & 2.88 & 33068 & 3.30 & 7.758 & 8.845 \\
\hline 2 & 14.016 & 40831938 & 97.12 & 969760 & 96.70 & 13.118 & 15.008 \\
\hline
\end{tabular}



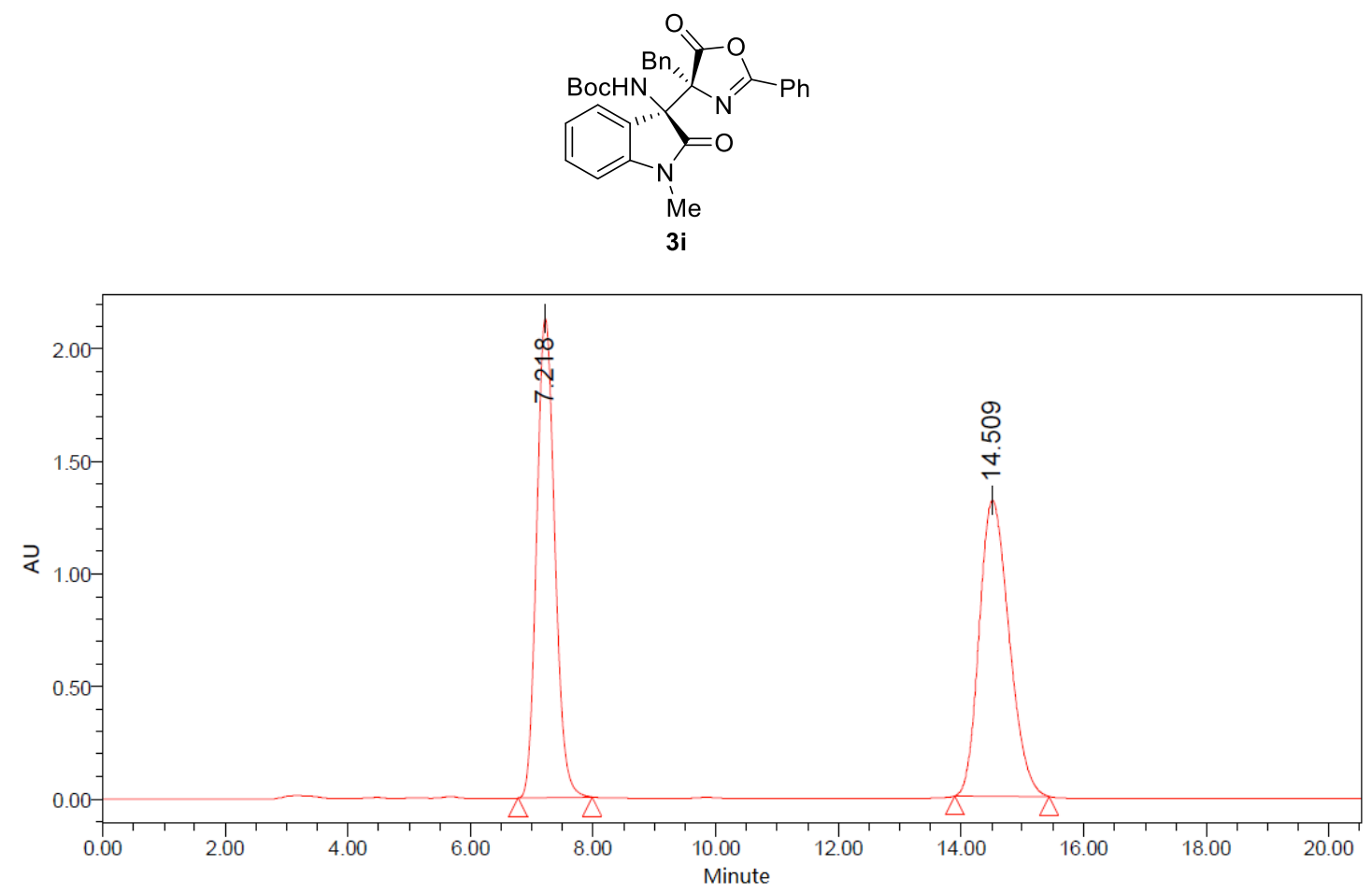

\begin{tabular}{|c|c|c|c|c|c|c|c|}
\hline & $\begin{array}{l}\mathbf{R T} \\
\text { (Min) }\end{array}$ & $\begin{array}{l}\text { Area } \\
\left(\mu U^{*} s\right)\end{array}$ & $\%$ Area & $\begin{array}{l}\text { He ight } \\
(\mu \mathrm{U})\end{array}$ & $\%$ Height & $\begin{array}{l}\text { Start Time } \\
\text { (Min) }\end{array}$ & $\begin{array}{l}\text { End Time } \\
\text { (Min) }\end{array}$ \\
\hline 1 & 7.218 & 43886975 & 49.68 & 2131861 & 61.85 & 6.778 & 7.990 \\
\hline 2 & 14.509 & 44460586 & 50.32 & 1314817 & 38.15 & 13.903 & 15.433 \\
\hline
\end{tabular}

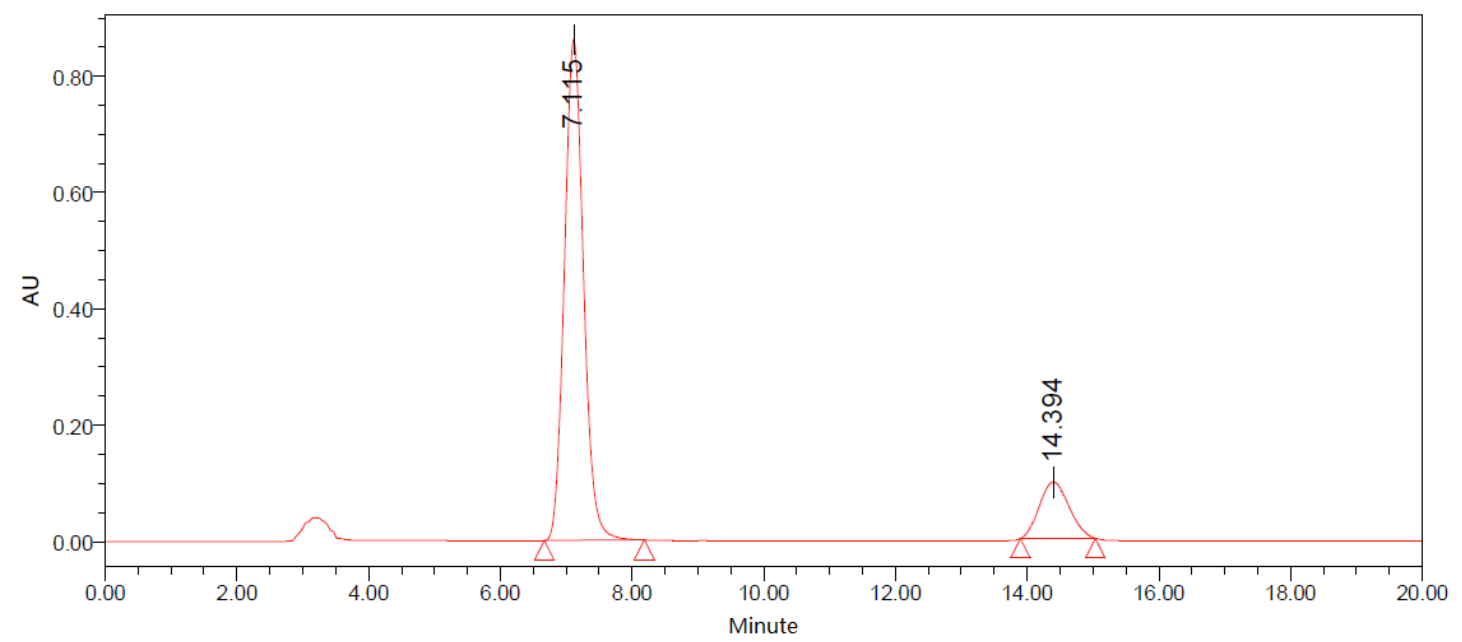

\begin{tabular}{|l|r|r|r|r|r|r|r|}
\hline & $\begin{array}{c}\text { RT } \\
(\text { Min) }\end{array}$ & \multicolumn{1}{|c|}{$\begin{array}{c}\text { Area } \\
\left(\mu U^{*}\right)\end{array}$} & $\%$ Area & $\begin{array}{c}\text { He ight } \\
(\mu \mathrm{U})\end{array}$ & $\%$ Height & $\begin{array}{c}\text { Start Time } \\
(\text { Min })\end{array}$ & $\begin{array}{c}\text { End Time } \\
(\text { Min })\end{array}$ \\
\hline 1 & 7.115 & 17367172 & 85.17 & 861155 & 89.90 & 6.672 & 8.183 \\
\hline 2 & 14.394 & 3024951 & 14.83 & 96801 & 10.10 & 13.897 & 15.032 \\
\hline
\end{tabular}



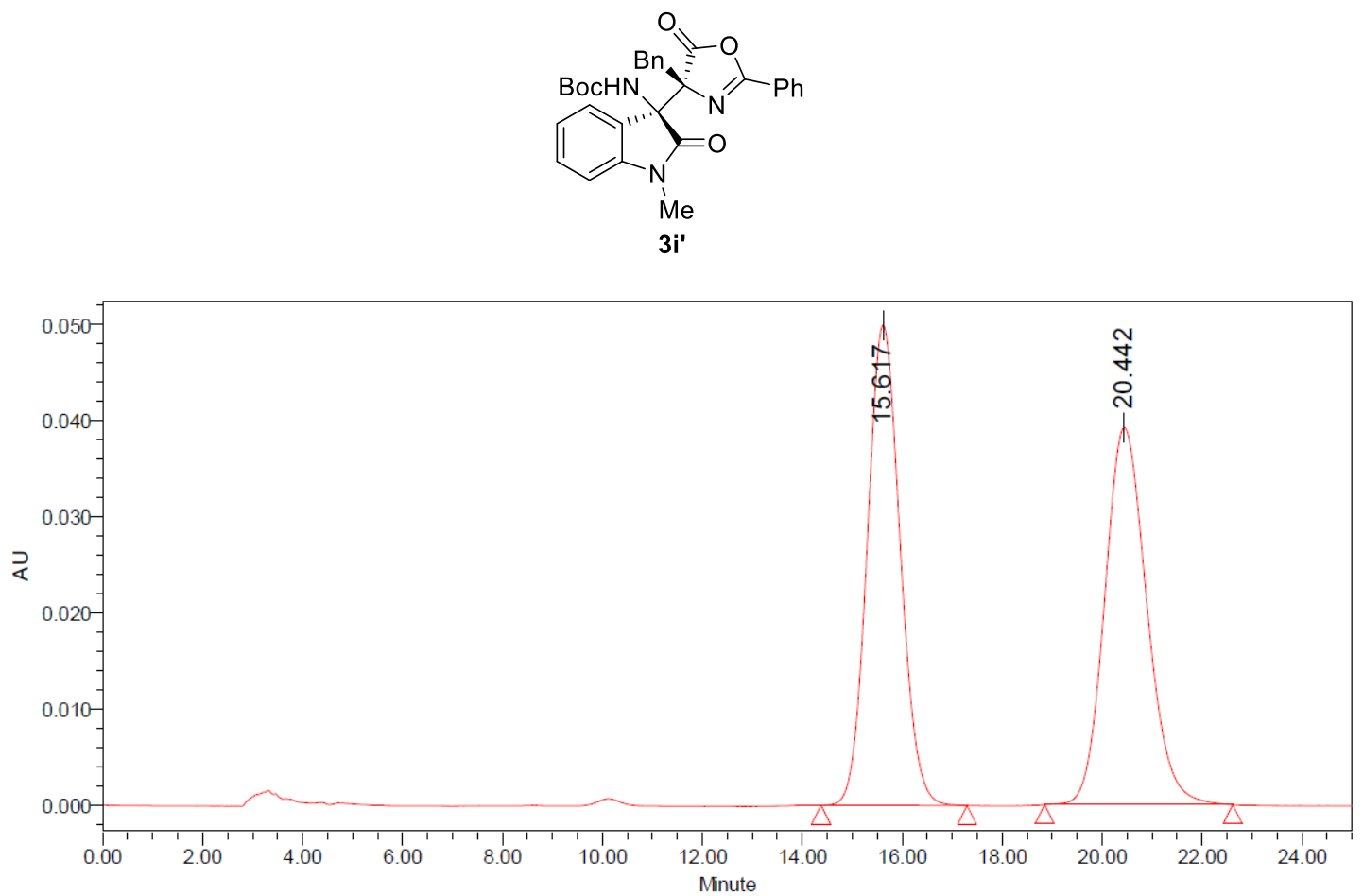

\begin{tabular}{|c|c|c|r|r|r|r|r|}
\hline & $\begin{array}{c}\text { RT } \\
(\text { Min) }\end{array}$ & $\begin{array}{c}\text { Area } \\
\left(\mu \mathrm{U}^{*} \mathrm{~s}\right)\end{array}$ & $\%$ Area & $\begin{array}{c}\text { He ight } \\
(\mu \mathrm{U})\end{array}$ & \% Height & $\begin{array}{c}\text { Start Time } \\
(\text { Min })\end{array}$ & $\begin{array}{c}\text { End Time } \\
(\mathrm{Min})\end{array}$ \\
\hline 1 & 15.617 & 2278771 & 49.87 & 49923 & 56.02 & 14.373 & 17.298 \\
\hline 2 & 20.442 & 2290748 & 50.13 & 39194 & 43.98 & 18.848 & 22.612 \\
\hline
\end{tabular}

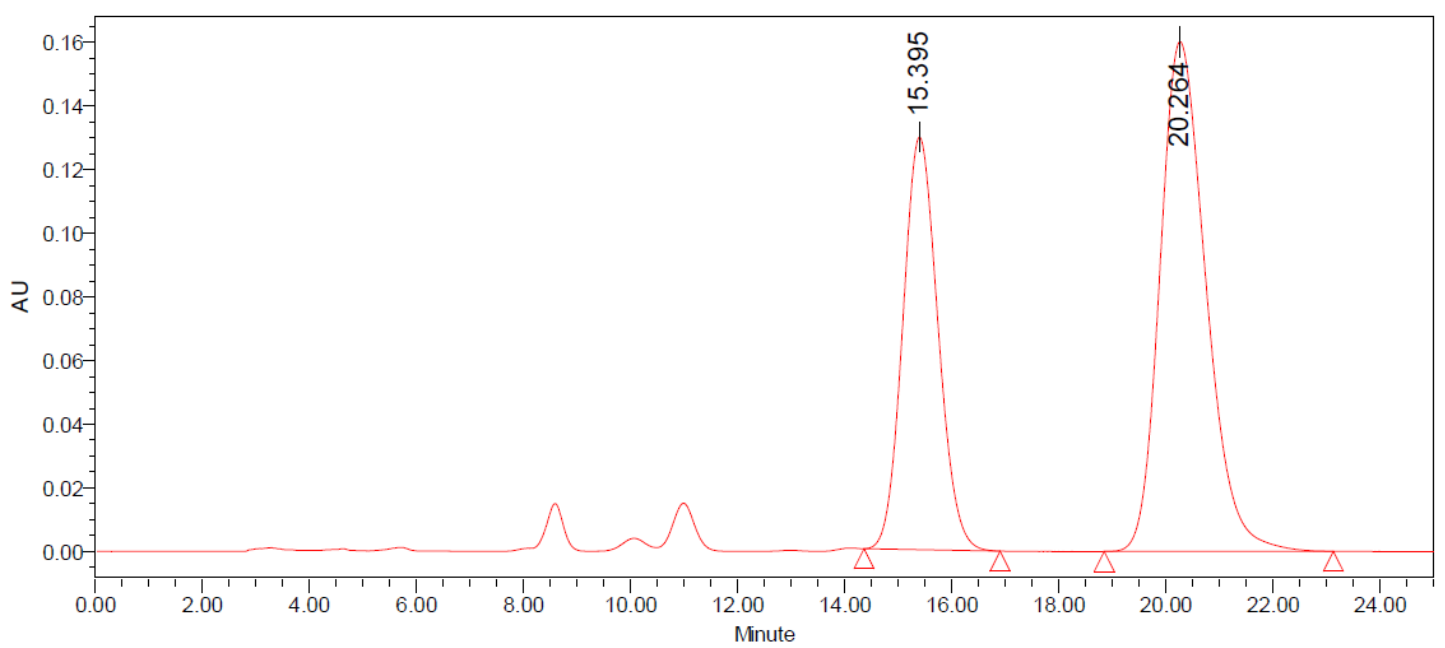

\begin{tabular}{|l|c|c|r|r|r|r|r|}
\hline & $\begin{array}{c}\mathrm{RT} \\
(\mathrm{Min})\end{array}$ & $\begin{array}{c}\text { Area } \\
\left(\mu \mathrm{U}{ }^{*} \mathrm{~s}\right)\end{array}$ & $\%$ Area & $\begin{array}{c}\text { He ight } \\
(\mu \mathrm{U})\end{array}$ & $\%$ Height & $\begin{array}{c}\text { Start Time } \\
(\text { Min) }\end{array}$ & $\begin{array}{c}\text { End Time } \\
(\mathrm{Min})\end{array}$ \\
\hline 1 & 15.395 & 5902455 & 38.22 & 129567 & 44.73 & 14.360 & 16.902 \\
\hline 2 & 20.264 & 9540693 & 61.78 & 160114 & 55.27 & 18.847 & 23.127 \\
\hline
\end{tabular}




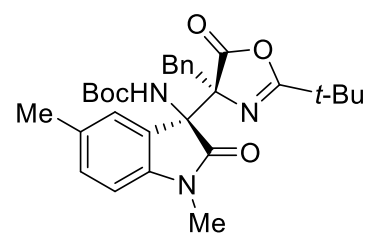

3j

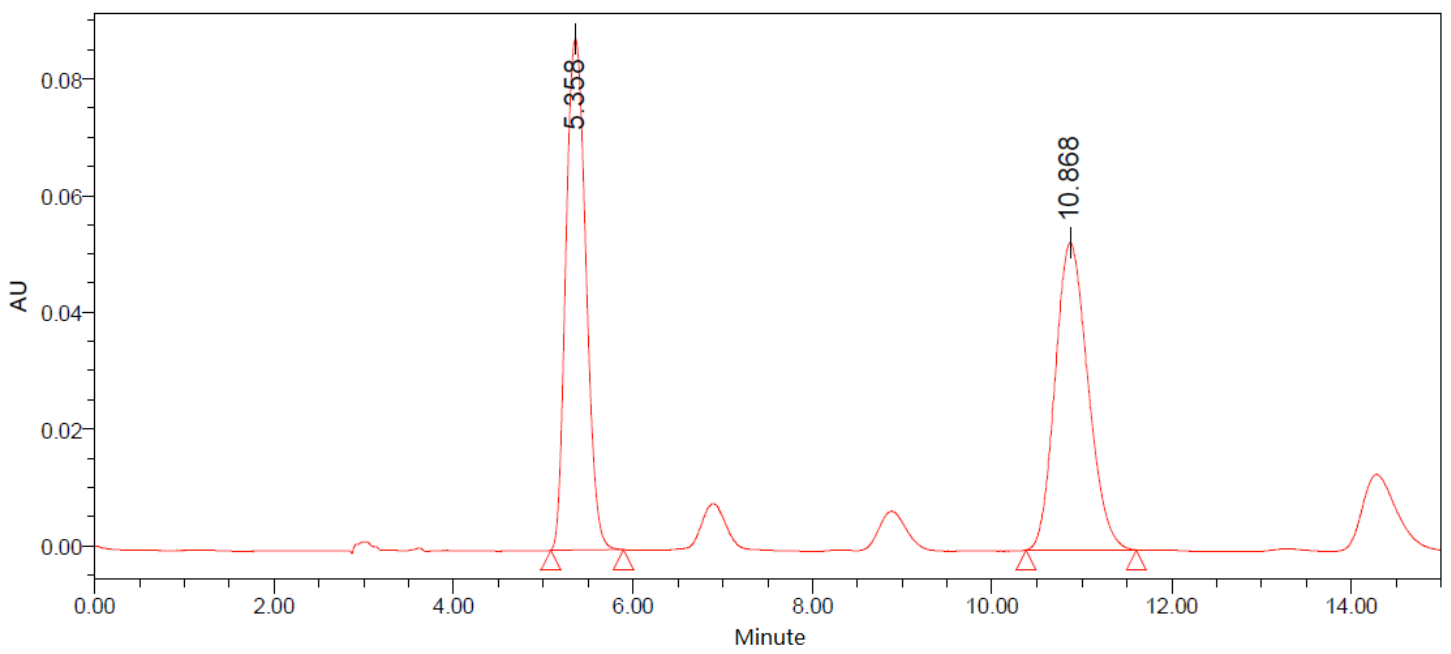

\begin{tabular}{|l|r|r|r|r|r|r|r|}
\hline & $\begin{array}{c}\text { RT } \\
(\text { Min) }\end{array}$ & \multicolumn{1}{c|}{$\begin{array}{c}\text { Area } \\
\left(\mu \mathrm{U}^{*} \mathrm{~s}\right)\end{array}$} & $\%$ Area & $\begin{array}{c}\text { He ight } \\
(\mu \mathrm{U})\end{array}$ & $\%$ Height & $\begin{array}{c}\text { Start Time } \\
(\text { Min) }\end{array}$ & $\begin{array}{r}\text { End Time } \\
(\text { Min) }\end{array}$ \\
\hline 1 & 5.358 & 1355903 & 50.27 & 87577 & 62.46 & 5.085 & 5.890 \\
\hline 2 & 10.868 & 1341205 & 49.73 & 52636 & 37.54 & 10.380 & 11.603 \\
\hline
\end{tabular}

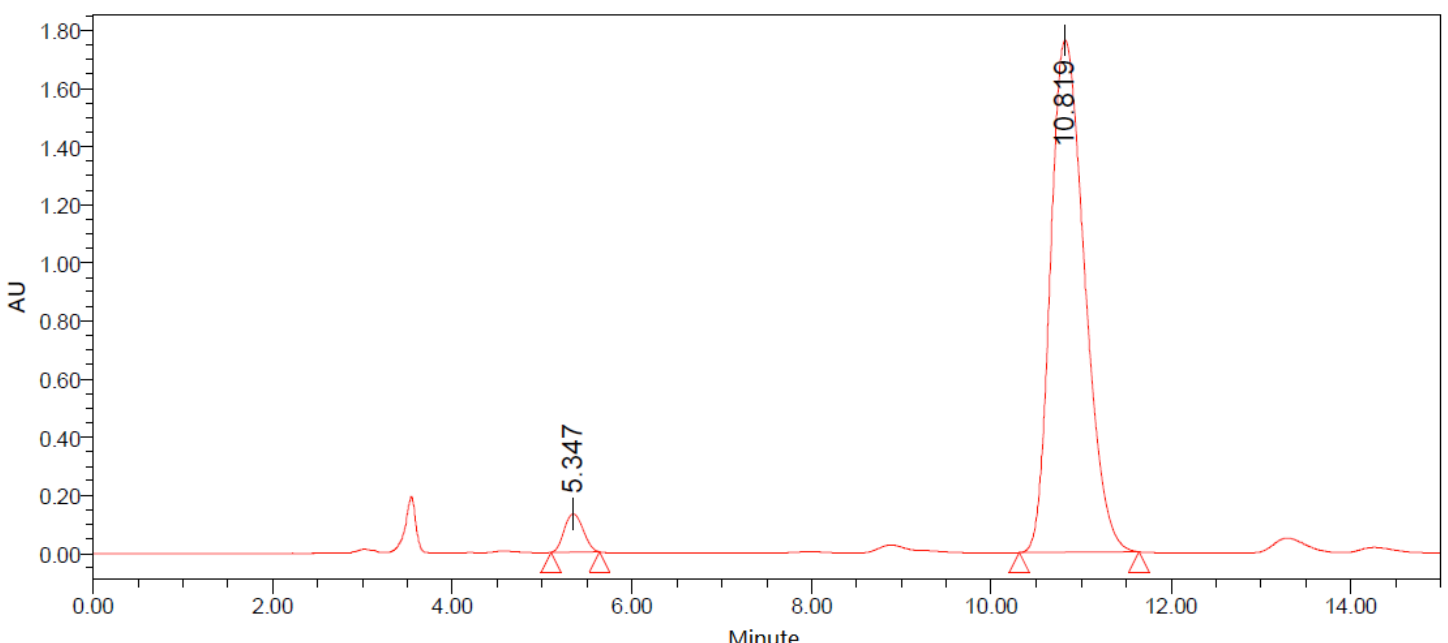

\begin{tabular}{|c|c|c|c|c|c|c|c|}
\hline & $\begin{array}{l}\text { RT } \\
\text { (Min) }\end{array}$ & $\begin{array}{l}\text { Area } \\
\left(\mu U^{\star} s\right)\end{array}$ & $\%$ Area & $\begin{array}{l}\text { He ight } \\
(\mu U)\end{array}$ & $\%$ Height & $\begin{array}{l}\text { Start Time } \\
\text { (Min) }\end{array}$ & $\begin{array}{l}\text { End Time } \\
\text { (Min) }\end{array}$ \\
\hline 1 & 5.347 & 1937001 & 4.00 & 131184 & 6.92 & 5.097 & 5.638 \\
\hline 2 & 10.819 & 46475535 & 96.00 & 1763400 & 93.08 & 10.310 & 11.642 \\
\hline
\end{tabular}



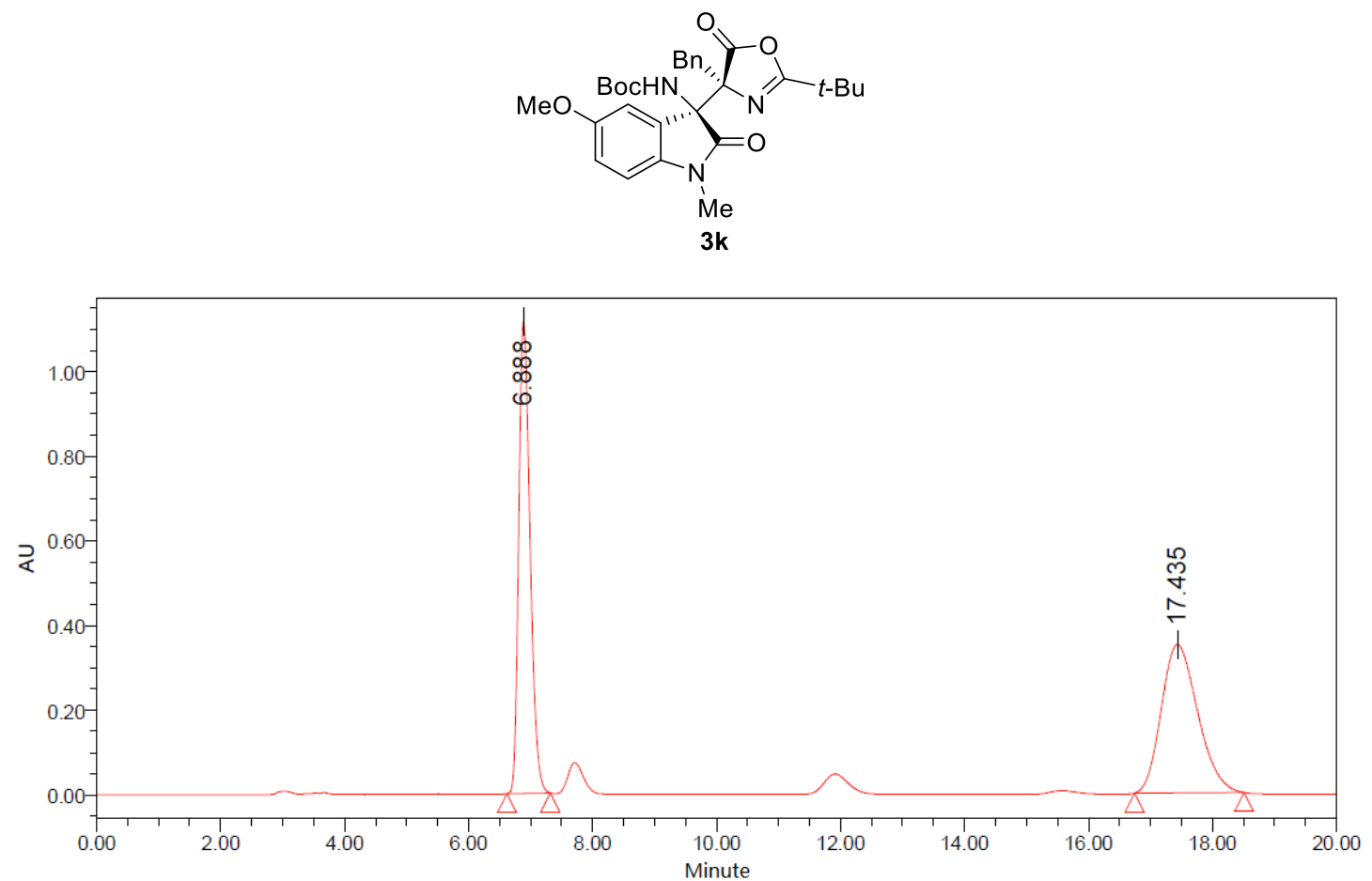

\begin{tabular}{|c|c|c|c|c|c|c|c|}
\hline & $\begin{array}{l}\text { RT } \\
\text { (Min) }\end{array}$ & $\begin{array}{l}\text { Area } \\
\left(\mu U^{*} s\right)\end{array}$ & $\%$ Area & $\begin{array}{l}\text { He ight } \\
(\mu U)\end{array}$ & $\%$ Height & $\begin{array}{l}\text { Start Time } \\
\text { (Min) }\end{array}$ & $\begin{array}{l}\text { End Time } \\
\text { (Min) }\end{array}$ \\
\hline 1 & 6.888 & 14184896 & 49.81 & 1116183 & 76.09 & 6.615 & 7.322 \\
\hline 2 & 17.435 & 14292781 & 50.19 & 350729 & 23.91 & 16.742 & 18.503 \\
\hline
\end{tabular}

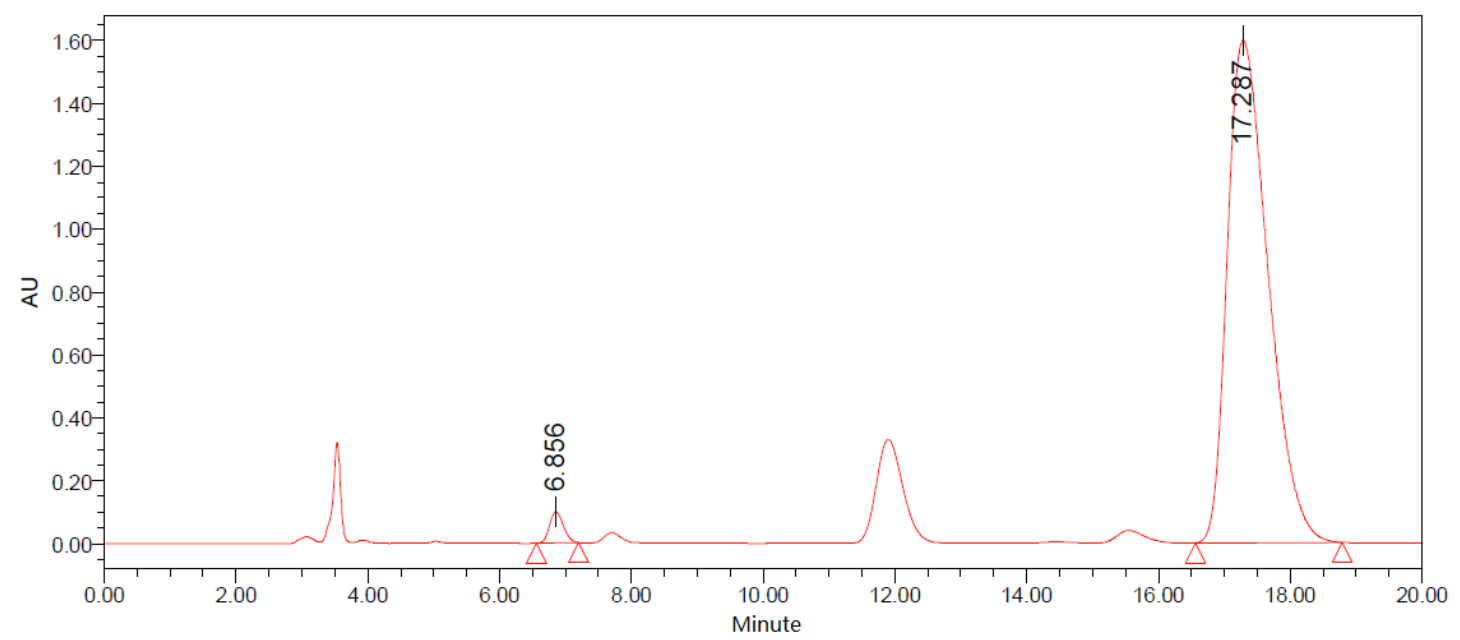

\begin{tabular}{|c|c|c|c|c|c|c|c|}
\hline & $\begin{array}{l}\text { RT } \\
\text { (Min) }\end{array}$ & $\begin{array}{l}\text { Area } \\
\left(m U^{*} s\right)\end{array}$ & $\%$ Area & $\begin{array}{l}\text { He ight } \\
(\mu U)\end{array}$ & $\%$ Height & $\begin{array}{l}\text { Start Time } \\
\text { (Min) }\end{array}$ & $\begin{array}{l}\text { End Time } \\
\text { (Min) }\end{array}$ \\
\hline 1 & 6.856 & 1465294 & 2.09 & 99877 & 5.88 & 6.555 & 7.202 \\
\hline 2 & 17.287 & 68735599 & 97.91 & 1597805 & 94.12 & 16.562 & 18.788 \\
\hline
\end{tabular}



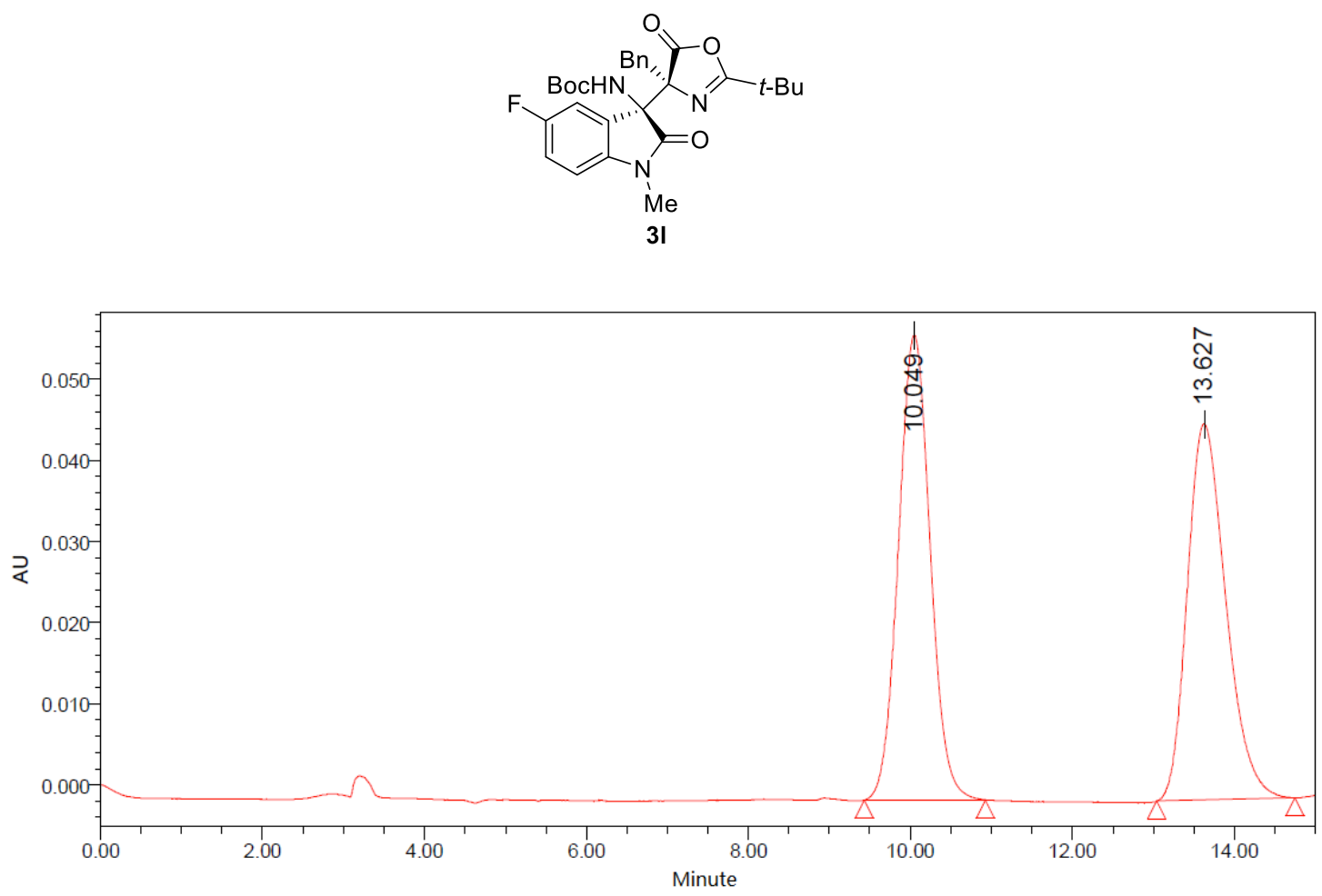

\begin{tabular}{|l|c|c|r|r|r|r|r|}
\hline & $\begin{array}{c}\text { RT } \\
(\text { Min) }\end{array}$ & $\begin{array}{c}\text { Area } \\
\left(\mu \mathrm{U}^{*} \mathrm{~s}\right)\end{array}$ & $\%$ Area & $\begin{array}{c}\text { He ight } \\
(\mu \mathrm{U})\end{array}$ & $\%$ Height & $\begin{array}{c}\text { Start Time } \\
(\text { Min) }\end{array}$ & $\begin{array}{c}\text { End Time } \\
(\text { Min) }\end{array}$ \\
\hline 1 & 10.049 & 1559792 & 50.53 & 57304 & 55.27 & 9.427 & 10.925 \\
\hline 2 & 13.627 & 1526904 & 49.47 & 46382 & 44.73 & 13.045 & 14.745 \\
\hline
\end{tabular}

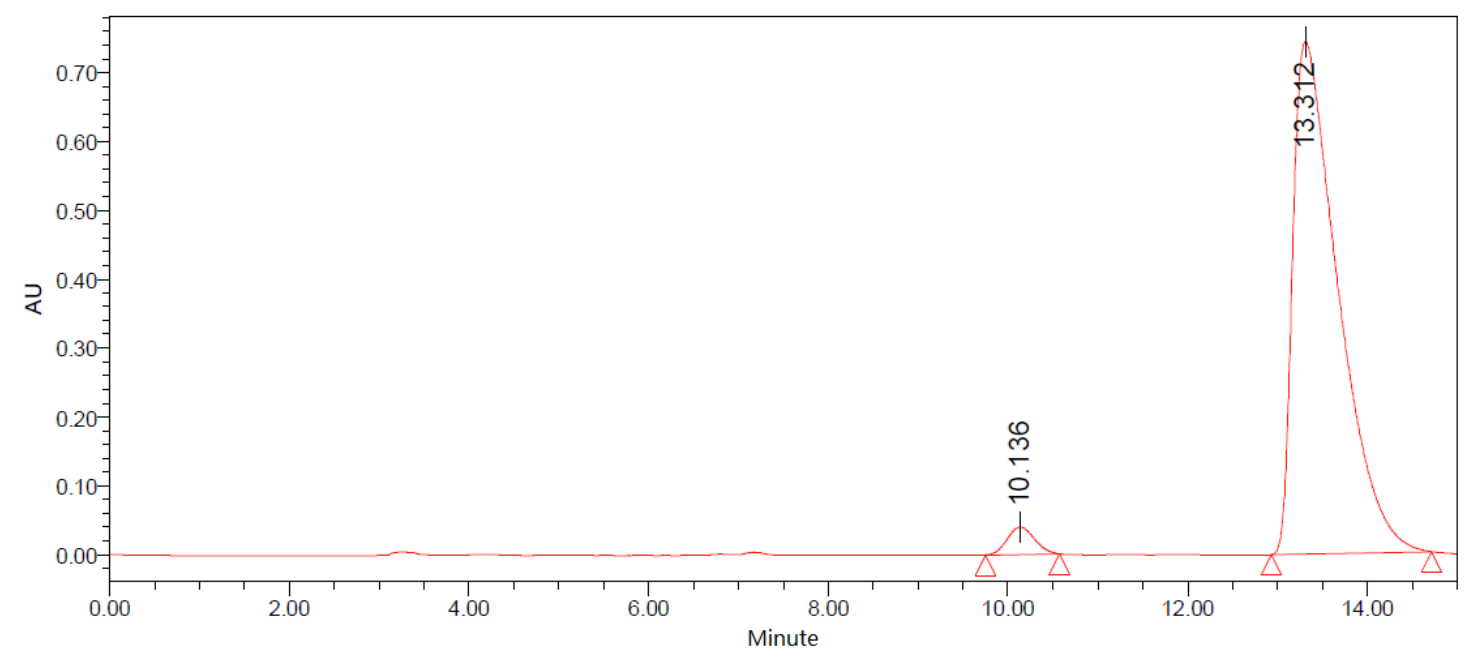

\begin{tabular}{|c|c|c|c|c|c|c|c|}
\hline & $\begin{array}{l}\mathbf{R T} \\
\text { (Min) }\end{array}$ & $\begin{array}{l}\text { Area } \\
\left(\mu U^{*} s\right)\end{array}$ & $\%$ Area & $\begin{array}{l}\text { He ight } \\
(\mu U)\end{array}$ & $\%$ Height & $\begin{array}{l}\text { Start Time } \\
\text { (Min) }\end{array}$ & $\begin{array}{l}\text { End Time } \\
\text { (Min) }\end{array}$ \\
\hline 1 & 10.136 & 835455 & 3.12 & 39848 & 5.08 & 9.747 & 10.570 \\
\hline 2 & 13.312 & 25910718 & 96.88 & 743876 & 94.92 & 12.928 & 14.712 \\
\hline
\end{tabular}



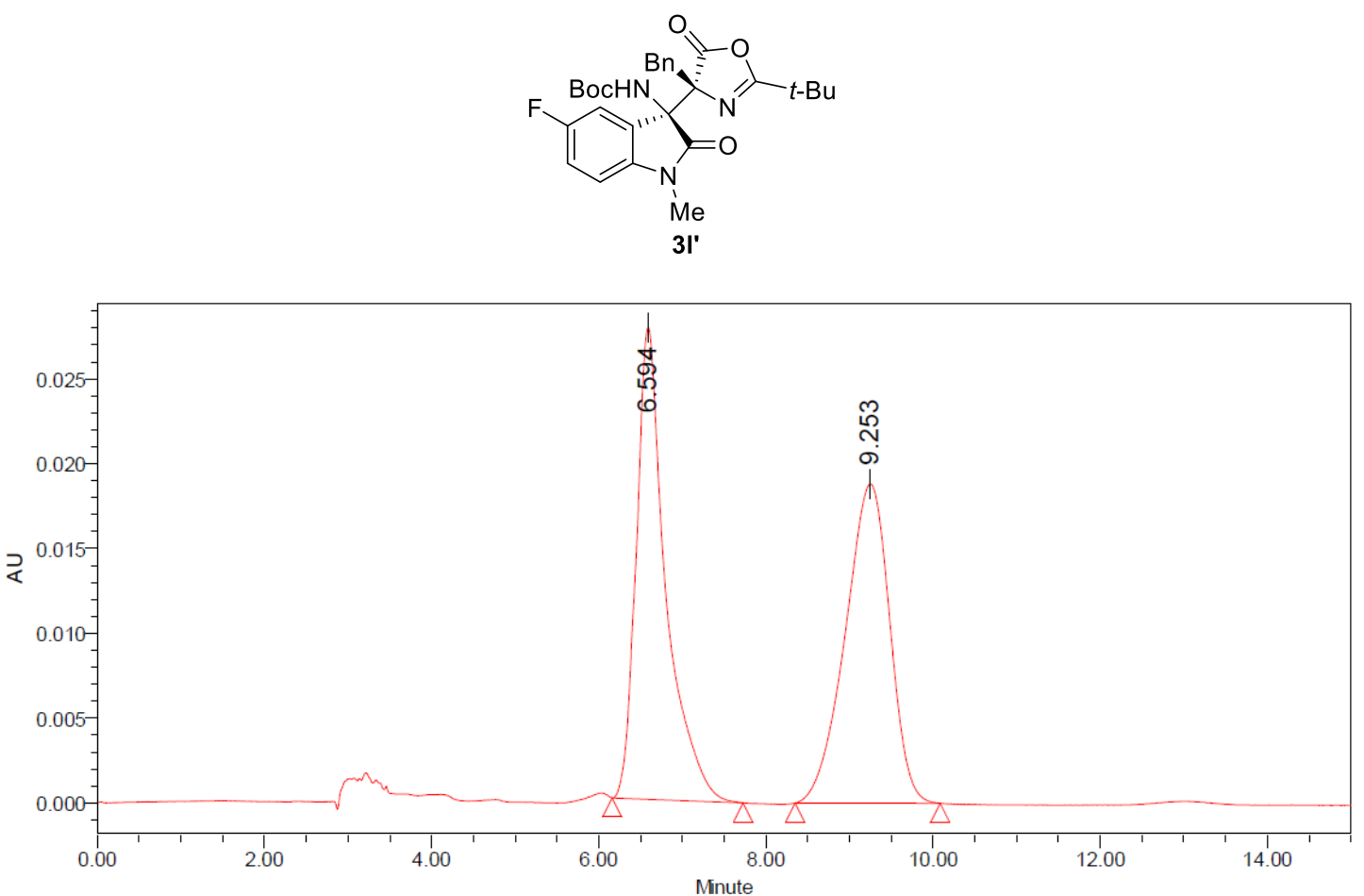

\begin{tabular}{|l|c|r|r|r|r|r|r|}
\hline & $\begin{array}{c}\mathrm{RT} \\
(\mathrm{Min})\end{array}$ & $\begin{array}{c}\text { Area } \\
\left(\mu \mathrm{U}^{*} \mathrm{~s}\right)\end{array}$ & $\%$ Area & $\begin{array}{l}\text { He ight } \\
(\mu \mathrm{U})\end{array}$ & $\%$ He ight & $\begin{array}{c}\text { Start Time } \\
(\text { Min) }\end{array}$ & $\begin{array}{c}\text { End Time } \\
(\text { Min) }\end{array}$ \\
\hline 1 & 6.594 & 684219 & 49.54 & 27832 & 59.66 & 6.160 & 7.727 \\
\hline 2 & 9.253 & 696832 & 50.46 & 18818 & 40.34 & 8.352 & 10.085 \\
\hline
\end{tabular}

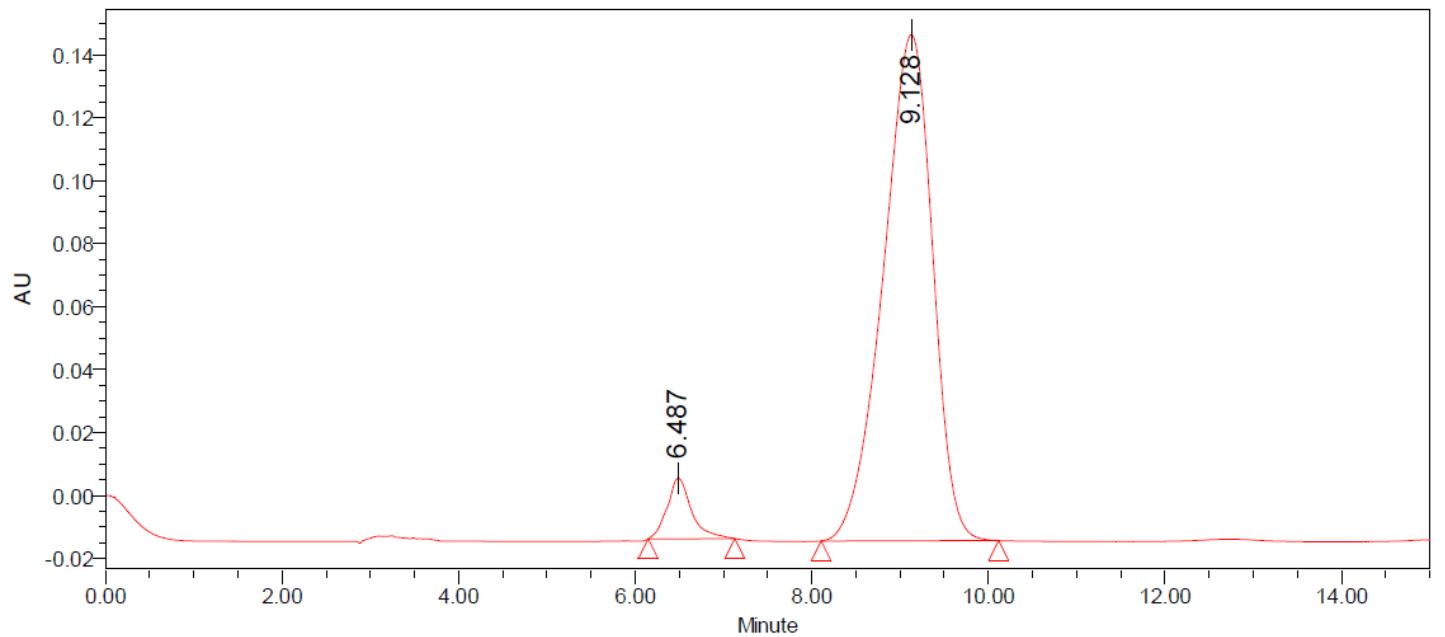

\begin{tabular}{|l|c|r|r|r|r|r|r|}
\hline & $\begin{array}{c}\mathbf{R T} \\
(\text { Min) }\end{array}$ & $\begin{array}{c}\text { Area } \\
\left(\mu \mathrm{U}{ }^{*} \mathrm{~s}\right)\end{array}$ & $\%$ Area & $\begin{array}{c}\text { He ight } \\
(\mu \mathrm{U})\end{array}$ & $\%$ He ight & $\begin{array}{c}\text { Start Time } \\
(\text { Min) }\end{array}$ & $\begin{array}{c}\text { End Time } \\
(\text { Min })\end{array}$ \\
\hline 1 & 6.487 & 368232 & 5.57 & 19278 & 10.71 & 6.147 & 7.127 \\
\hline 2 & 9.128 & 6241377 & 94.43 & 160665 & 89.29 & 8.105 & 10.117 \\
\hline
\end{tabular}



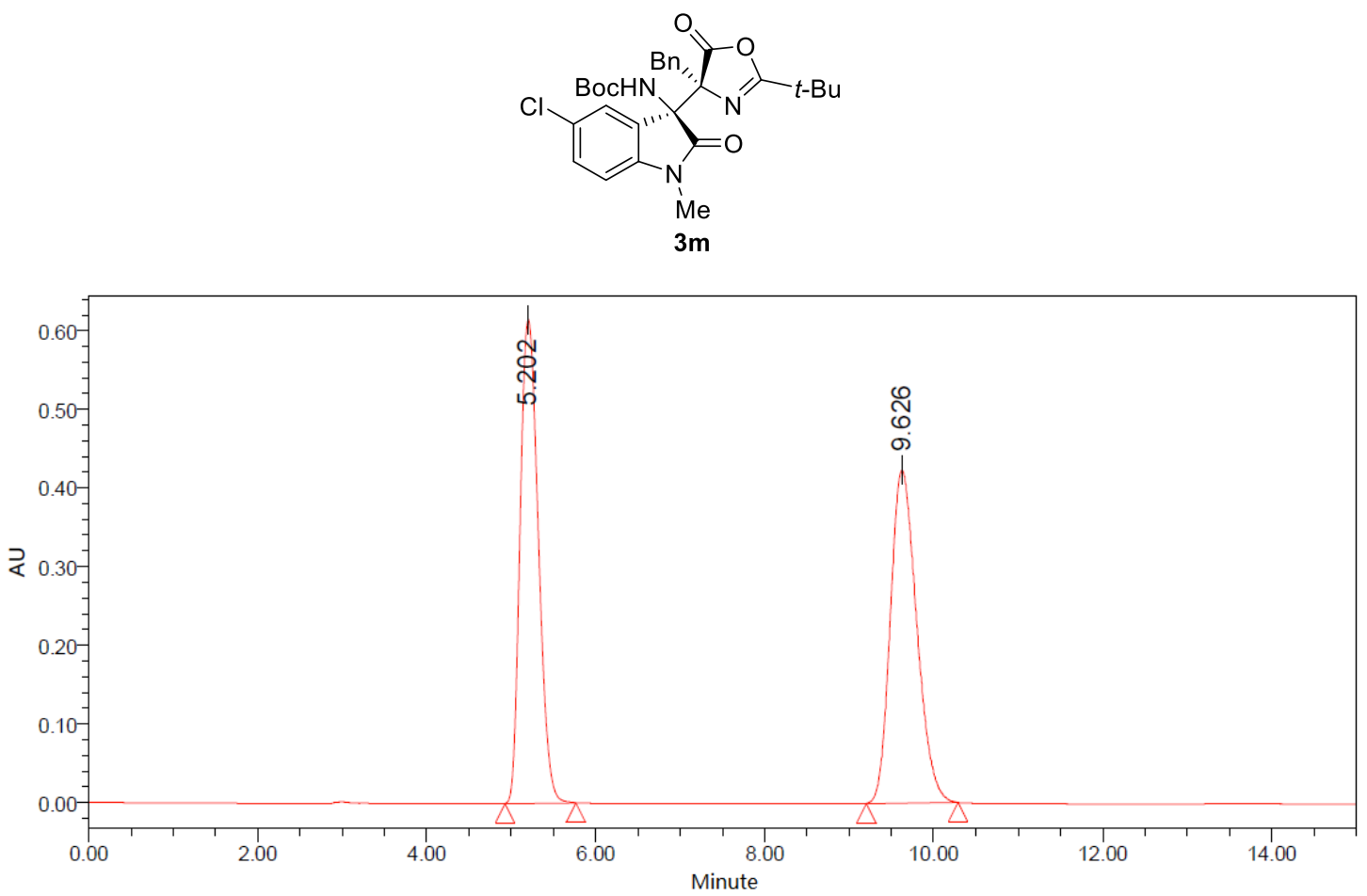

\begin{tabular}{|l|c|c|r|r|r|r|r|}
\hline & $\begin{array}{c}\text { RT } \\
(\text { Min) }\end{array}$ & $\begin{array}{c}\text { Area } \\
\left(\mu \mathrm{U}^{*} \mathrm{~s}\right)\end{array}$ & $\%$ Area & $\begin{array}{c}\text { He ight } \\
(\mu \mathrm{U})\end{array}$ & \% Height & $\begin{array}{c}\text { Start Time } \\
(\text { Min) }\end{array}$ & $\begin{array}{r}\text { End Time } \\
(\mathrm{Min})\end{array}$ \\
\hline 1 & 5.202 & 9195782 & 50.12 & 614902 & 59.22 & 4.927 & 5.762 \\
\hline 2 & 9.626 & 9152336 & 49.88 & 423496 & 40.78 & 9.205 & 10.288 \\
\hline
\end{tabular}

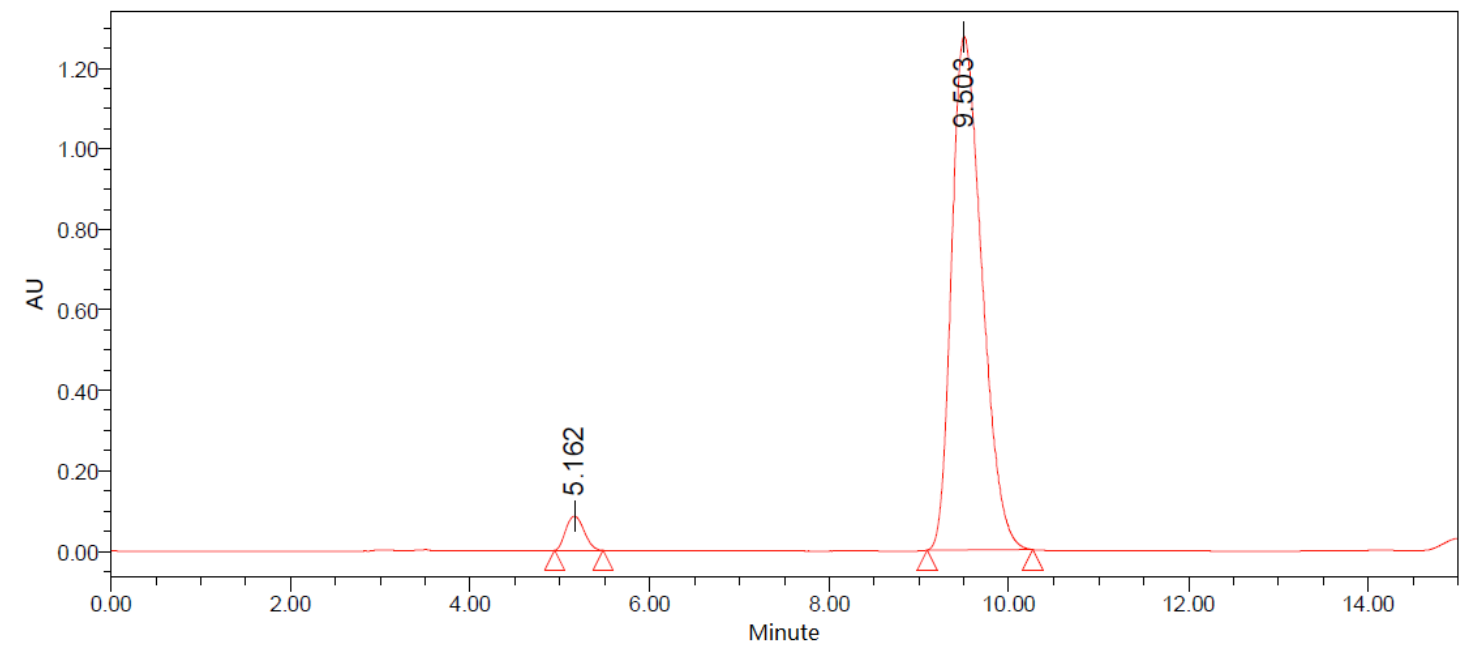

\begin{tabular}{|l|c|r|r|r|r|r|r|}
\hline & $\begin{array}{c}\text { RT } \\
(\text { Min) }\end{array}$ & \multicolumn{1}{|c|}{$\begin{array}{c}\text { Area } \\
\left(\mu \mathrm{U}{ }^{*} \mathrm{~s}\right)\end{array}$} & \multicolumn{1}{c|}{$\%$ Area } & $\begin{array}{c}\text { He ight } \\
(\mu \mathrm{U})\end{array}$ & $\%$ He ight & $\begin{array}{l}\text { Start Time } \\
(\text { Min })\end{array}$ & $\begin{array}{c}\text { End Time } \\
(\text { Min })\end{array}$ \\
\hline 1 & 5.162 & 1216113 & 3.91 & 85083 & 6.25 & 4.938 & 5.480 \\
\hline 2 & 9.503 & 29894767 & 96.09 & 1275734 & 93.75 & 9.092 & 10.265 \\
\hline
\end{tabular}



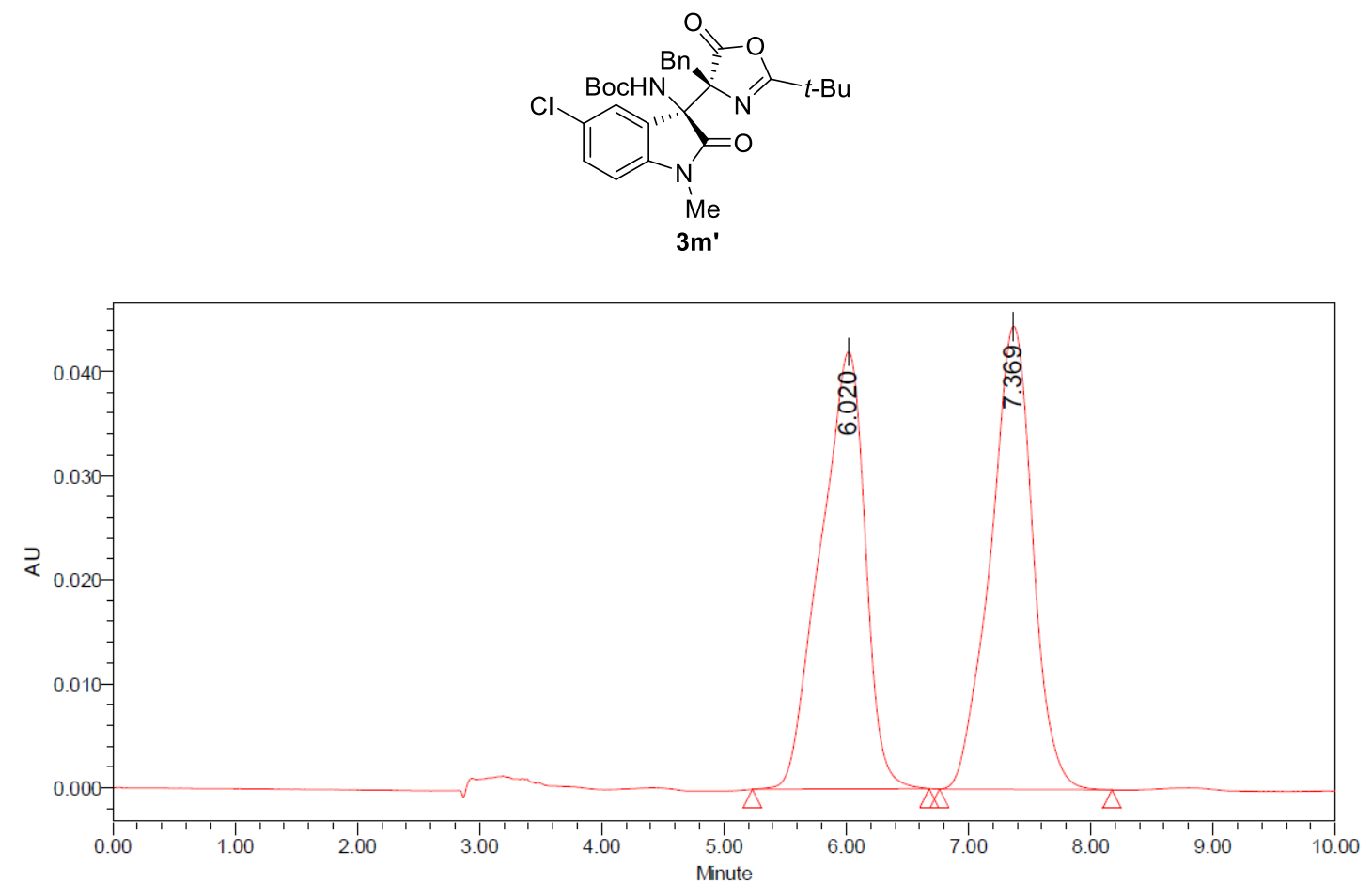

\begin{tabular}{|l|c|c|r|r|r|r|r|}
\hline & $\begin{array}{c}\text { RT } \\
(\text { Min) }\end{array}$ & $\begin{array}{c}\text { Area } \\
\left(\mu U^{*} \mathrm{~s}\right)\end{array}$ & $\%$ Area & $\begin{array}{c}\text { He ight } \\
(\mu \mathrm{U})\end{array}$ & $\%$ He ight & $\begin{array}{c}\text { Start Time } \\
(\text { Min) }\end{array}$ & $\begin{array}{r}\text { End Time } \\
(\text { Min) }\end{array}$ \\
\hline 1 & 6.020 & 1067001 & 49.93 & 41996 & 48.57 & 5.232 & 6.678 \\
\hline 2 & 7.369 & 1070082 & 50.07 & 44476 & 51.43 & 6.763 & 8.173 \\
\hline
\end{tabular}

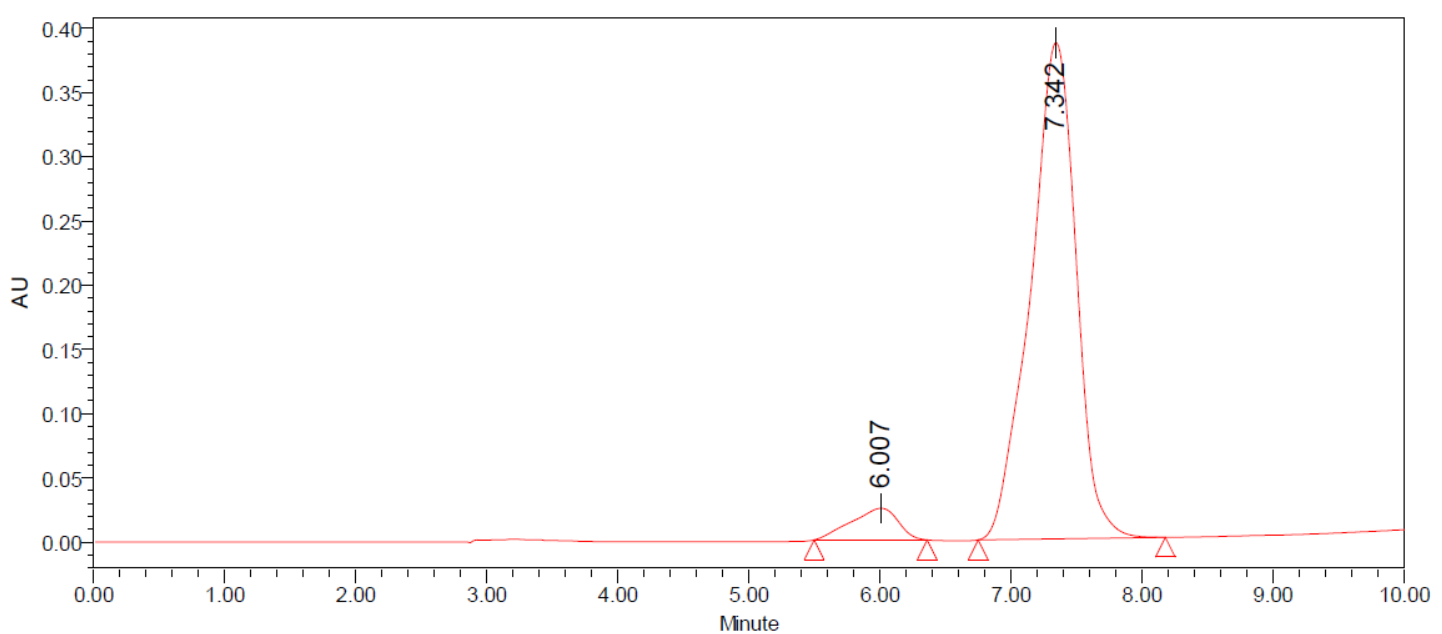

\begin{tabular}{|l|c|r|r|r|r|r|r|}
\hline & $\begin{array}{c}\text { RT } \\
(\text { Min) }\end{array}$ & \multicolumn{1}{|c|}{$\begin{array}{c}\text { Area } \\
\left(\mu \mathrm{U}^{*} \mathrm{~s}\right)\end{array}$} & \% Area & $\begin{array}{c}\text { He ight } \\
(\mu \mathrm{U})\end{array}$ & $\%$ Height & $\begin{array}{c}\text { Start Time } \\
(\text { Min) }\end{array}$ & $\begin{array}{c}\text { End Time } \\
(\text { Min) }\end{array}$ \\
\hline 1 & 6.007 & 610262 & 6.14 & 24625 & 5.99 & 5.497 & 6.358 \\
\hline 2 & 7.342 & 9333575 & 93.86 & 386519 & 94.01 & 6.748 & 8.177 \\
\hline
\end{tabular}



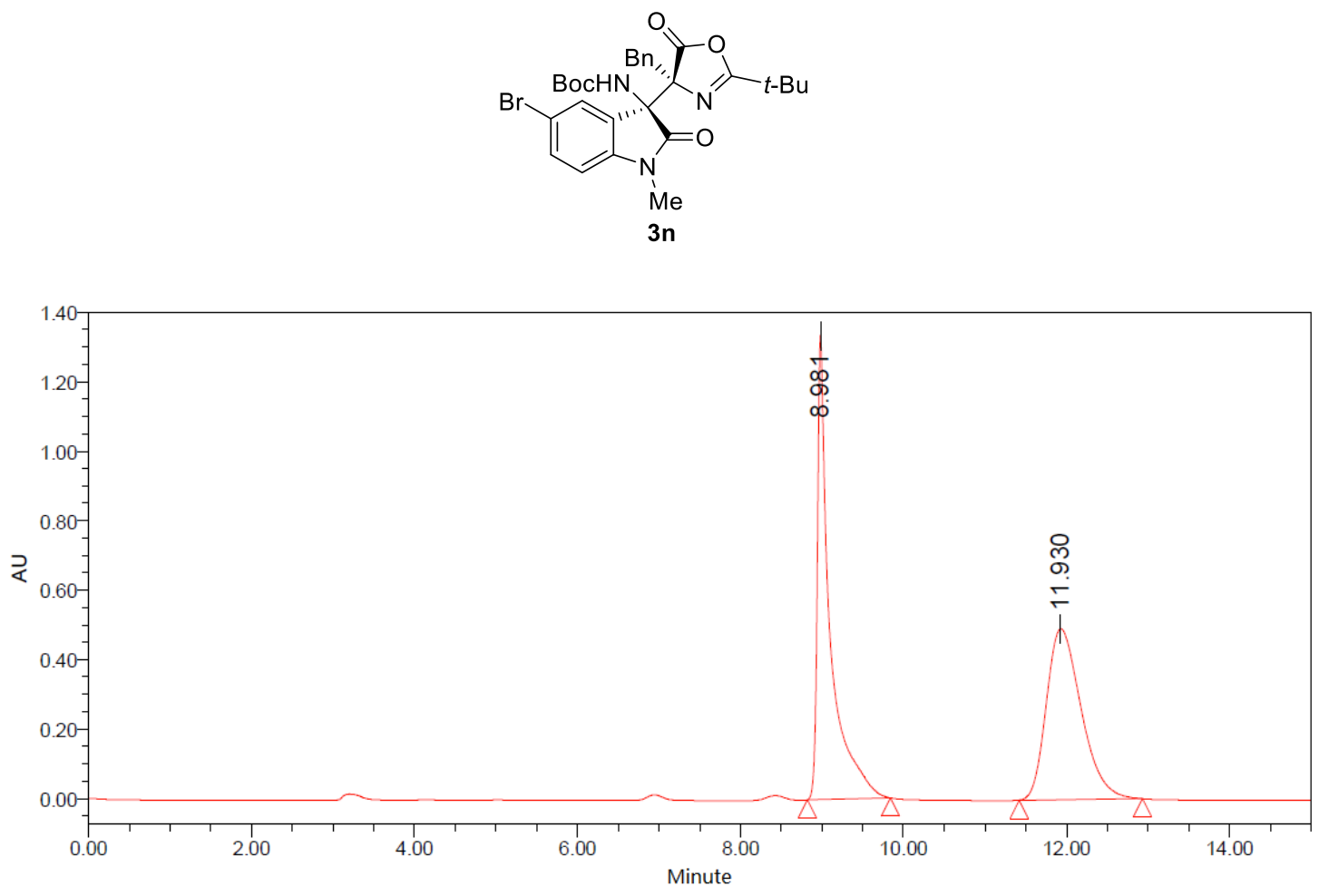

\begin{tabular}{|c|c|c|c|c|c|c|c|}
\hline & $\begin{array}{l}\text { RT } \\
\text { (Min) }\end{array}$ & $\underset{\left(\mu U^{*} s\right)}{\text { Area }}$ & $\%$ Area & $\begin{array}{l}\text { He ight } \\
(\mu U)\end{array}$ & $\%$ Height & $\begin{array}{l}\text { Start Time } \\
\text { (Min) }\end{array}$ & $\begin{array}{l}\text { End Time } \\
\text { (Min) }\end{array}$ \\
\hline 1 & 8.981 & 14447038 & 49.63 & 1337921 & 73.10 & 8.822 & 9.837 \\
\hline 2 & 11.930 & 14661051 & 50.37 & 492444 & 26.90 & 11.417 & 12.928 \\
\hline
\end{tabular}

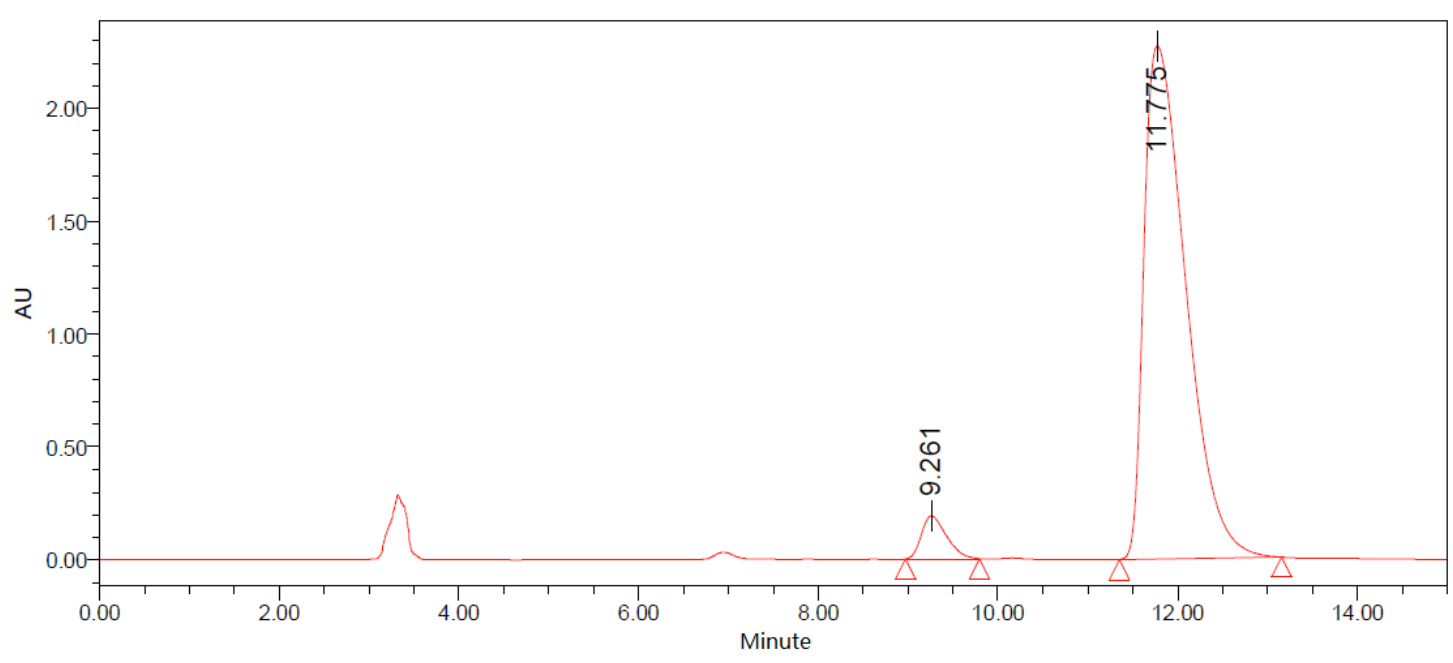

\begin{tabular}{|c|c|c|c|c|c|c|c|}
\hline & $\begin{array}{l}\mathbf{R T} \\
\text { (Min) }\end{array}$ & $\begin{array}{c}\text { Area } \\
\left(\mu U^{*} s\right)\end{array}$ & $\%$ Area & $\begin{array}{l}\text { He ight } \\
(\mu \mathrm{U})\end{array}$ & $\%$ Height & $\begin{array}{l}\text { Start Time } \\
\text { (Min) }\end{array}$ & $\begin{array}{l}\text { End Time } \\
\text { (Min) }\end{array}$ \\
\hline 1 & 9.261 & 3686540 & 4.76 & 190377 & 7.73 & 8.968 & 9.792 \\
\hline 2 & 11.775 & 73683596 & 95.24 & 2271321 & 92.27 & 11.350 & 13.155 \\
\hline
\end{tabular}



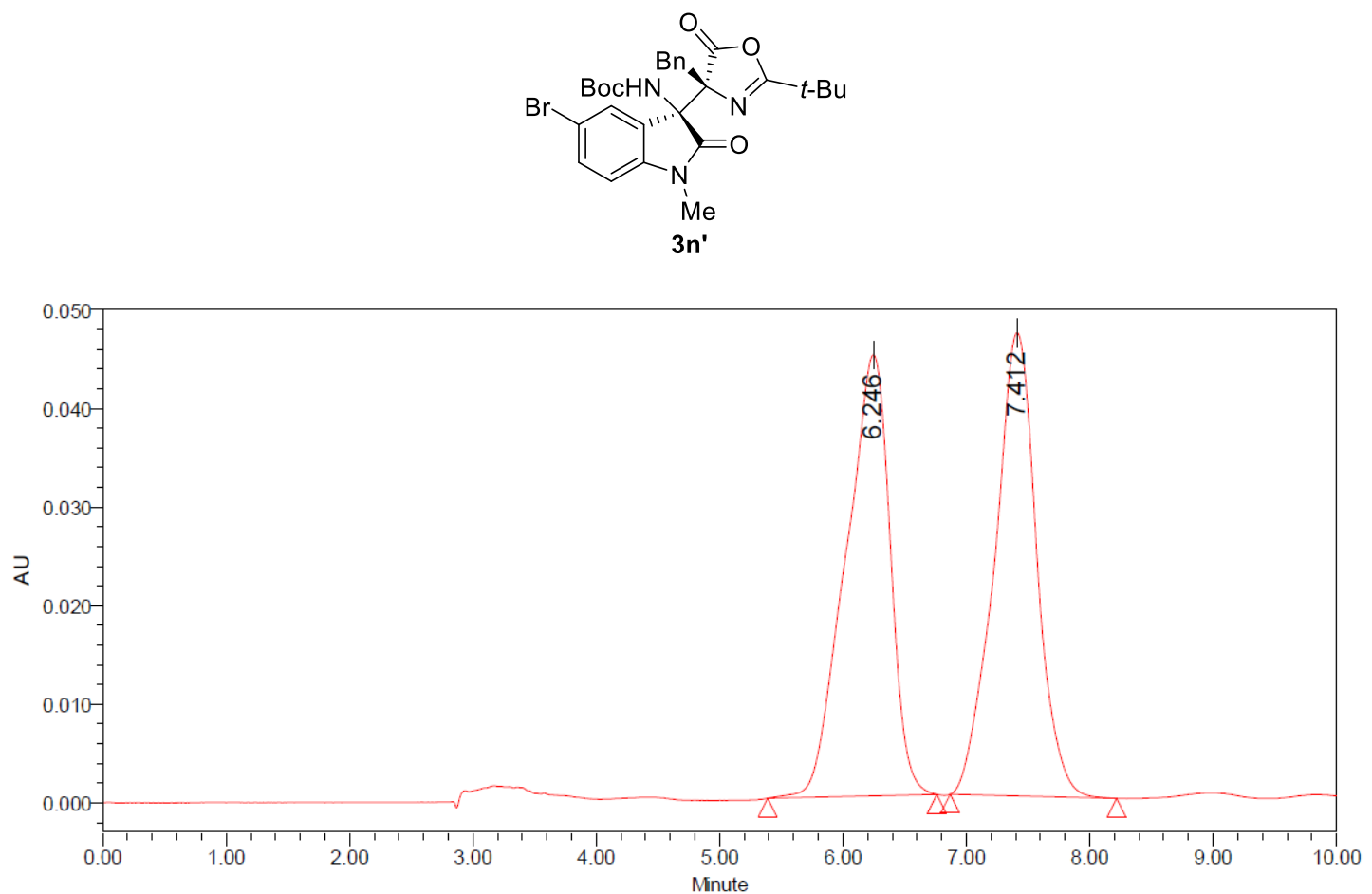

\begin{tabular}{|l|c|c|r|r|r|r|r|}
\hline & $\begin{array}{c}\mathbf{R} \\
(\mathrm{Min})\end{array}$ & $\begin{array}{c}\text { Area } \\
\left(\mu \mathrm{U}^{*} \mathrm{~s}\right)\end{array}$ & $\%$ Area & $\begin{array}{c}\text { He ight } \\
(\mu \mathrm{U})\end{array}$ & $\%$ He ight & $\begin{array}{c}\text { Start Time } \\
(\text { Min) }\end{array}$ & $\begin{array}{r}\text { End Time } \\
(\mathrm{Min})\end{array}$ \\
\hline 1 & 6.246 & 1119342 & 50.02 & 44726 & 48.78 & 5.388 & 6.760 \\
\hline 2 & 7.412 & 1118581 & 49.98 & 46972 & 51.22 & 6.868 & 8.217 \\
\hline
\end{tabular}

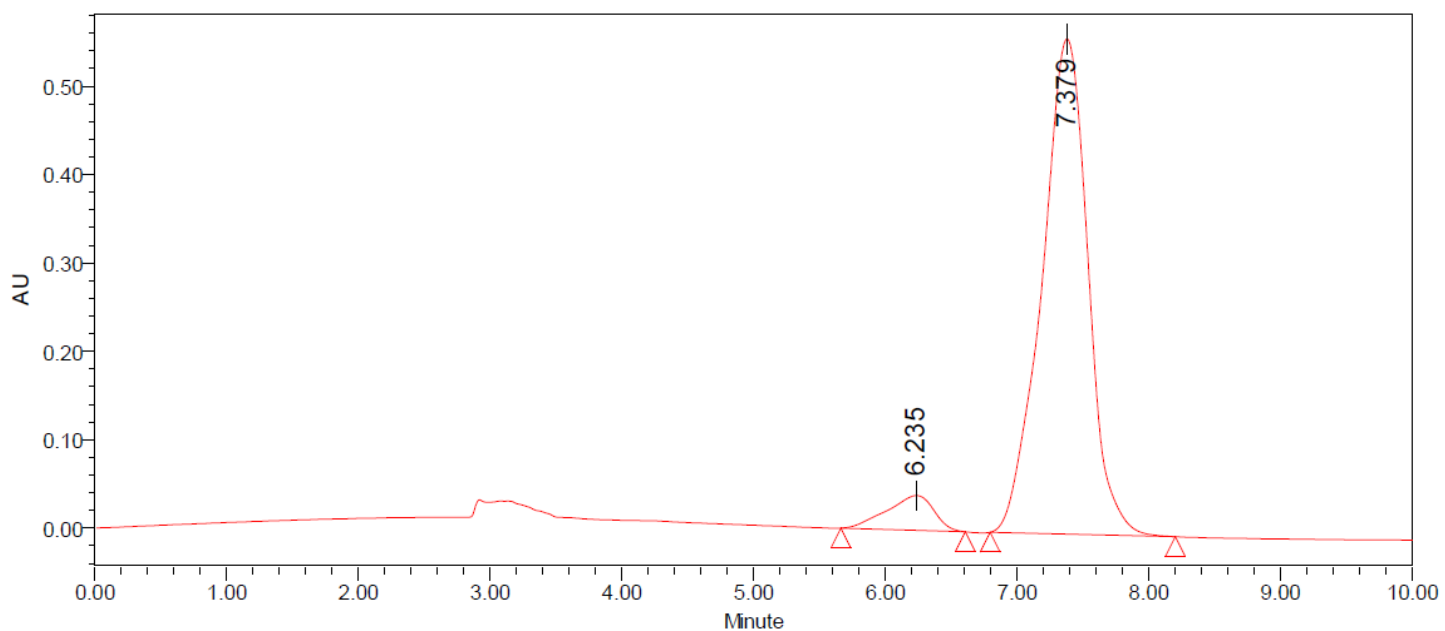

\begin{tabular}{|l|c|r|r|r|r|r|r|}
\hline & $\begin{array}{c}\text { RT } \\
(\text { Min) }\end{array}$ & \multicolumn{1}{c|}{$\begin{array}{c}\text { Area } \\
\left(\mu \mathrm{U}^{*} \mathrm{~s}\right)\end{array}$} & $\%$ Area & $\begin{array}{c}\text { He ight } \\
(\mu \mathrm{U})\end{array}$ & $\%$ Height & $\begin{array}{c}\text { Start Time } \\
(\text { Min) }\end{array}$ & $\begin{array}{c}\text { End Time } \\
(\text { Min) }\end{array}$ \\
\hline 1 & 6.235 & 975154 & 6.69 & 39390 & 6.57 & 5.662 & 6.607 \\
\hline 2 & 7.379 & 13594571 & 93.31 & 560303 & 93.43 & 6.795 & 8.200 \\
\hline
\end{tabular}



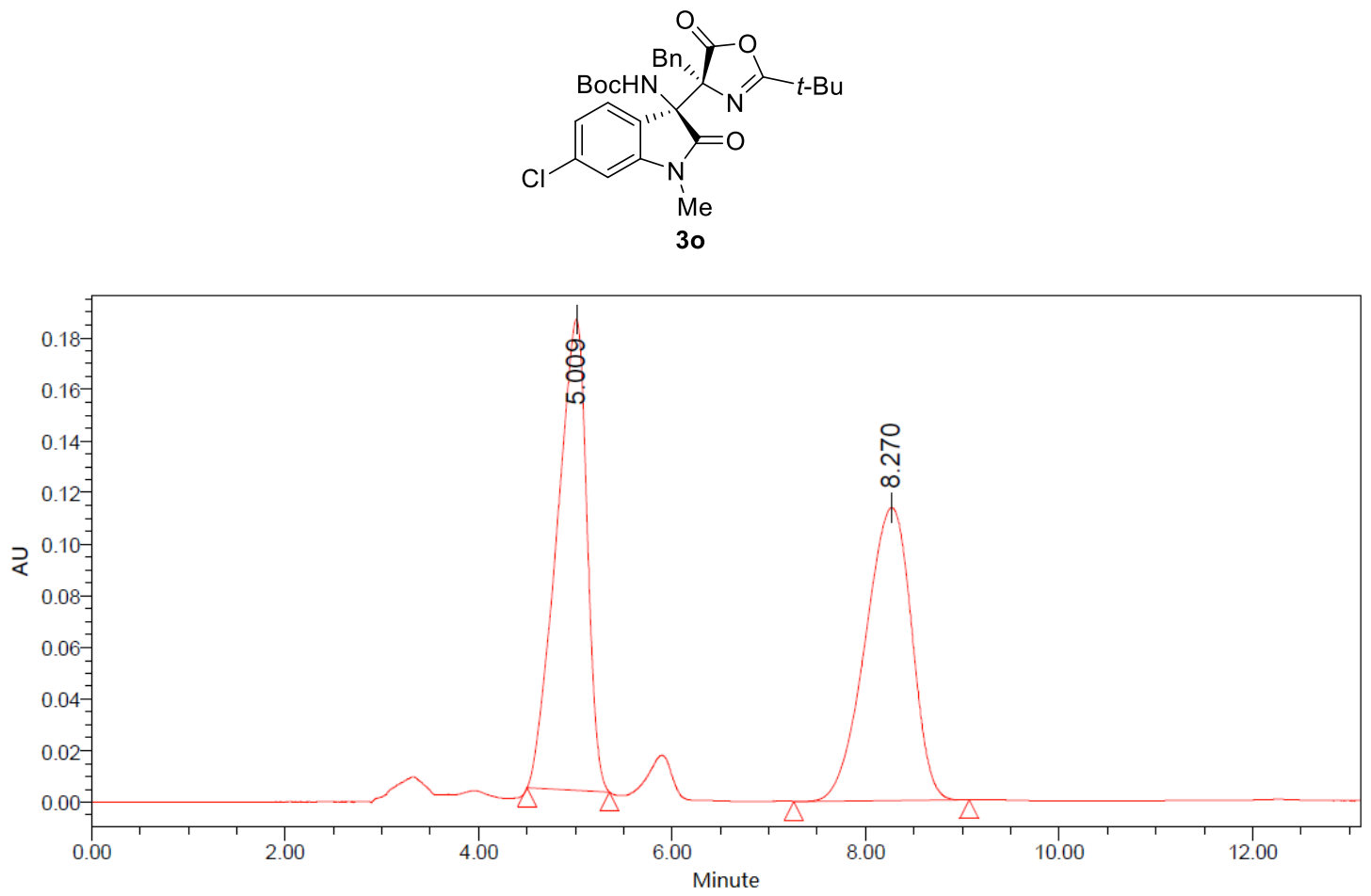

\begin{tabular}{|l|c|c|r|r|r|r|r|}
\hline & $\begin{array}{c}\text { RT } \\
(\text { Min })\end{array}$ & $\begin{array}{c}\text { Area } \\
\left(\mu U^{*} \mathrm{~s}\right)\end{array}$ & $\%$ Area & $\begin{array}{c}\text { He ight } \\
(\mu \mathrm{U})\end{array}$ & $\%$ Height & $\begin{array}{c}\text { Start Time } \\
(\text { Min) }\end{array}$ & $\begin{array}{c}\text { End Time } \\
(\text { Min) }\end{array}$ \\
\hline 1 & 5.009 & 3955911 & 51.15 & 182584 & 61.67 & 4.500 & 5.352 \\
\hline 2 & 8.270 & 3778626 & 48.85 & 113489 & 38.33 & 7.257 & 9.070 \\
\hline
\end{tabular}

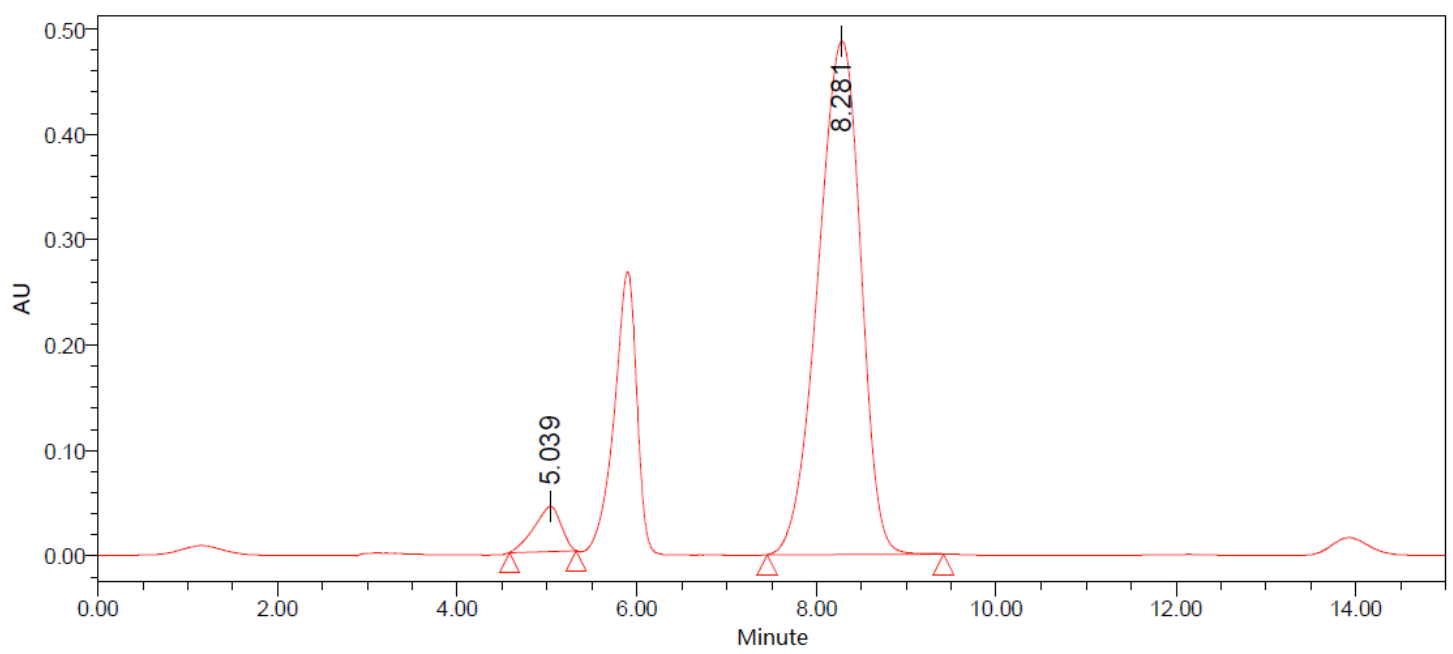

\begin{tabular}{|c|c|c|c|c|c|c|c|}
\hline & $\begin{array}{l}\mathbf{R T} \\
\text { (Min) }\end{array}$ & $\begin{array}{c}\text { Area } \\
\left(\mu U^{*} s\right)\end{array}$ & $\%$ Area & $\begin{array}{l}\text { He ight } \\
(\mu U)\end{array}$ & $\%$ Height & $\begin{array}{l}\text { Start Time } \\
\text { (Min) }\end{array}$ & $\begin{array}{l}\text { End Time } \\
\text { (Min) }\end{array}$ \\
\hline 1 & 5.039 & 893120 & 5.25 & 42709 & 8.06 & 4.578 & 5.323 \\
\hline 2 & 8.281 & 16118673 & 94.75 & 487281 & 91.94 & 7.448 & 9.415 \\
\hline
\end{tabular}



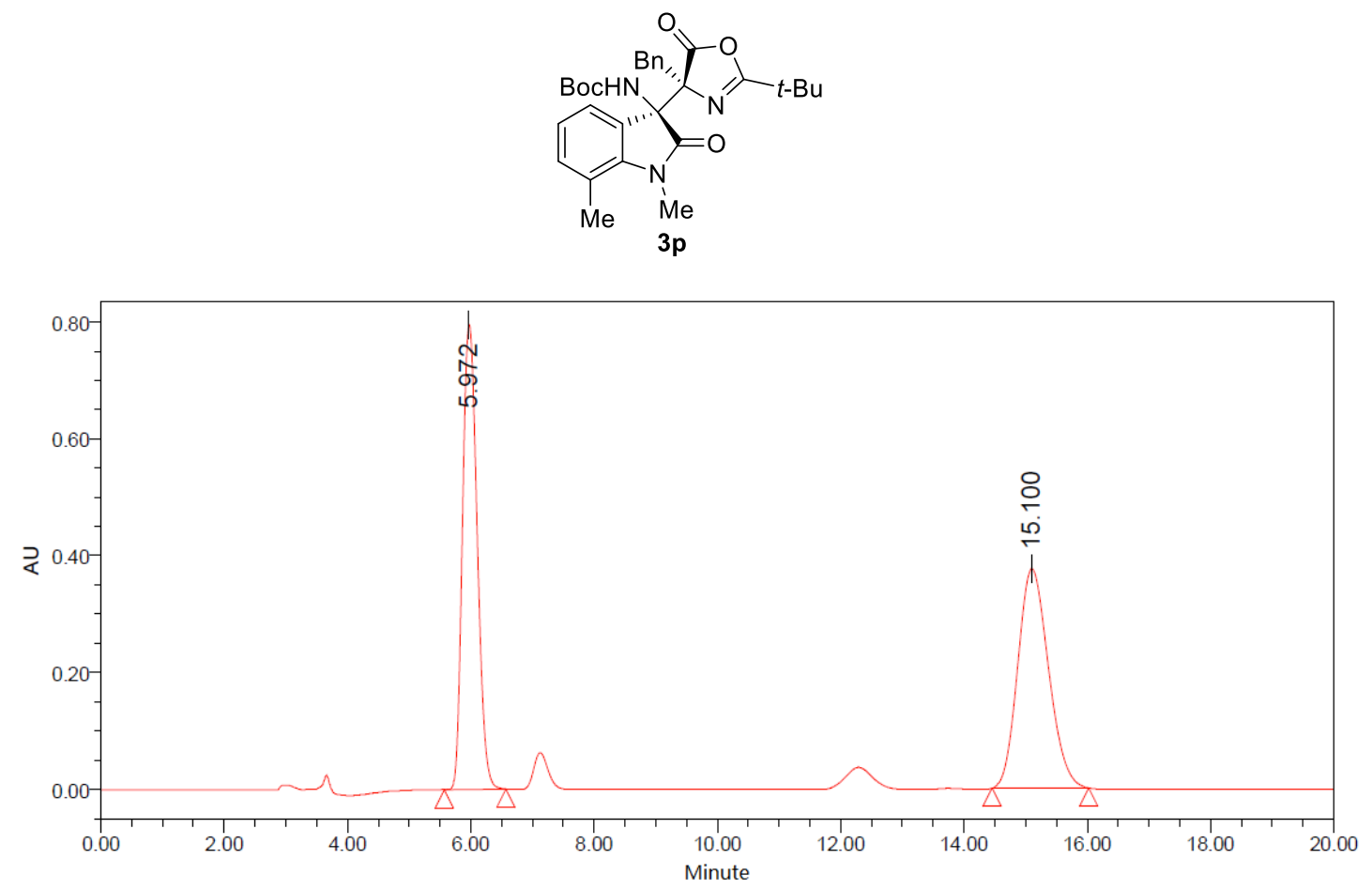

\begin{tabular}{|l|c|c|r|r|r|r|r|}
\hline & $\begin{array}{c}\text { RT } \\
(\text { Min) }\end{array}$ & $\begin{array}{c}\text { Area } \\
\left(\mu U^{*} \mathrm{~s}\right)\end{array}$ & \multicolumn{1}{c|}{ \% Area } & $\begin{array}{c}\text { Height } \\
(\mu \mathrm{U})\end{array}$ & $\%$ Height & $\begin{array}{c}\text { Start Time } \\
(\text { Min) }\end{array}$ & $\begin{array}{c}\text { End Time } \\
(\text { Min) }\end{array}$ \\
\hline 1 & 5.972 & 13119174 & 50.22 & 794703 & 67.93 & 5.577 & 6.570 \\
\hline 2 & 15.100 & 13004020 & 49.78 & 375235 & 32.07 & 14.453 & 16.020 \\
\hline
\end{tabular}

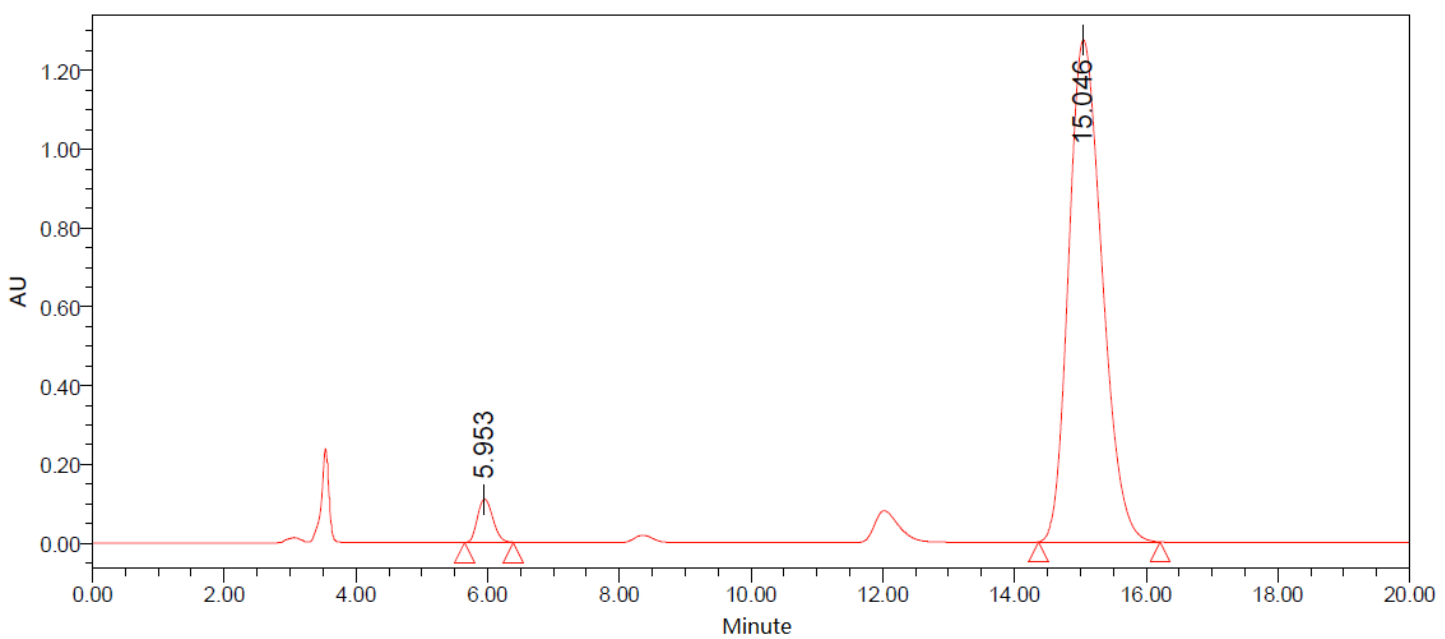

\begin{tabular}{|c|c|c|c|c|c|c|c|}
\hline & $\begin{array}{l}\text { RT } \\
\text { (Min) }\end{array}$ & $\begin{array}{l}\text { Area } \\
\left(\mu U U^{*} s\right)\end{array}$ & $\%$ Area & $\begin{array}{l}\text { He ight } \\
(\mu U)\end{array}$ & $\%$ Height & $\begin{array}{l}\text { Start Time } \\
\text { (Min) }\end{array}$ & $\begin{array}{l}\text { End Time } \\
\text { (Min) }\end{array}$ \\
\hline 1 & 5.953 & 1798405 & 3.84 & 110008 & 7.94 & 5.652 & 6.390 \\
\hline 2 & 15.046 & 45065708 & 96.16 & 1275583 & 92.06 & 14.365 & 16.215 \\
\hline
\end{tabular}



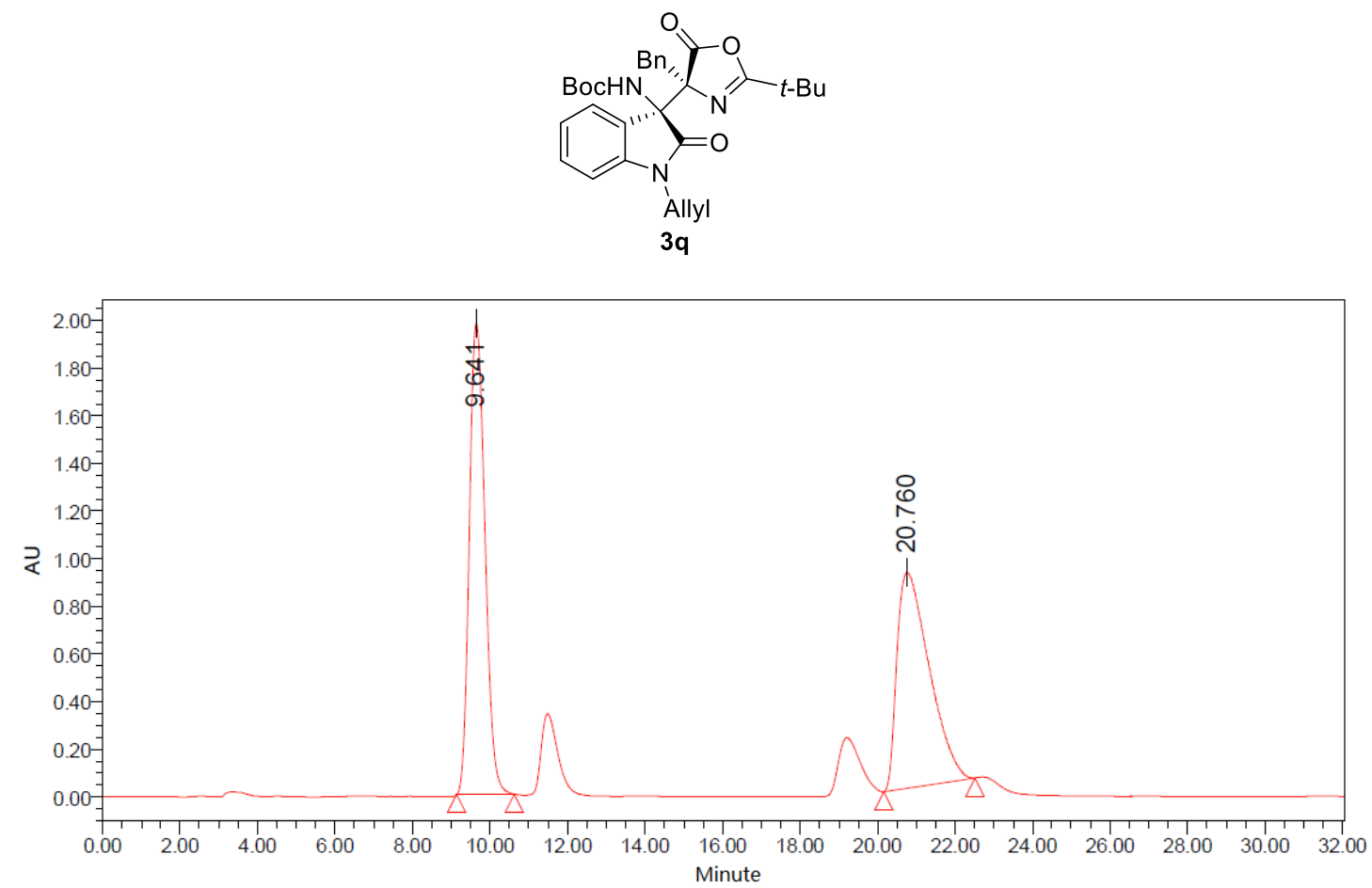

\begin{tabular}{|l|r|c|r|r|r|r|r|}
\hline & $\begin{array}{c}\text { RT } \\
(\text { Min) }\end{array}$ & $\begin{array}{c}\text { Area } \\
\left(\mu U^{*} \mathrm{~s}\right)\end{array}$ & $\%$ Area & $\begin{array}{c}\text { Height } \\
(\mu \mathrm{U})\end{array}$ & \% Height & $\begin{array}{c}\text { Start Time } \\
(\text { Min) }\end{array}$ & $\begin{array}{c}\text { End Time } \\
(\mathrm{Min})\end{array}$ \\
\hline 1 & 9.641 & 55446133 & 51.48 & 1978019 & 68.60 & 9.142 & 10.620 \\
\hline 2 & 20.760 & 52260082 & 48.52 & 905236 & 31.40 & 20.153 & 22.502 \\
\hline
\end{tabular}

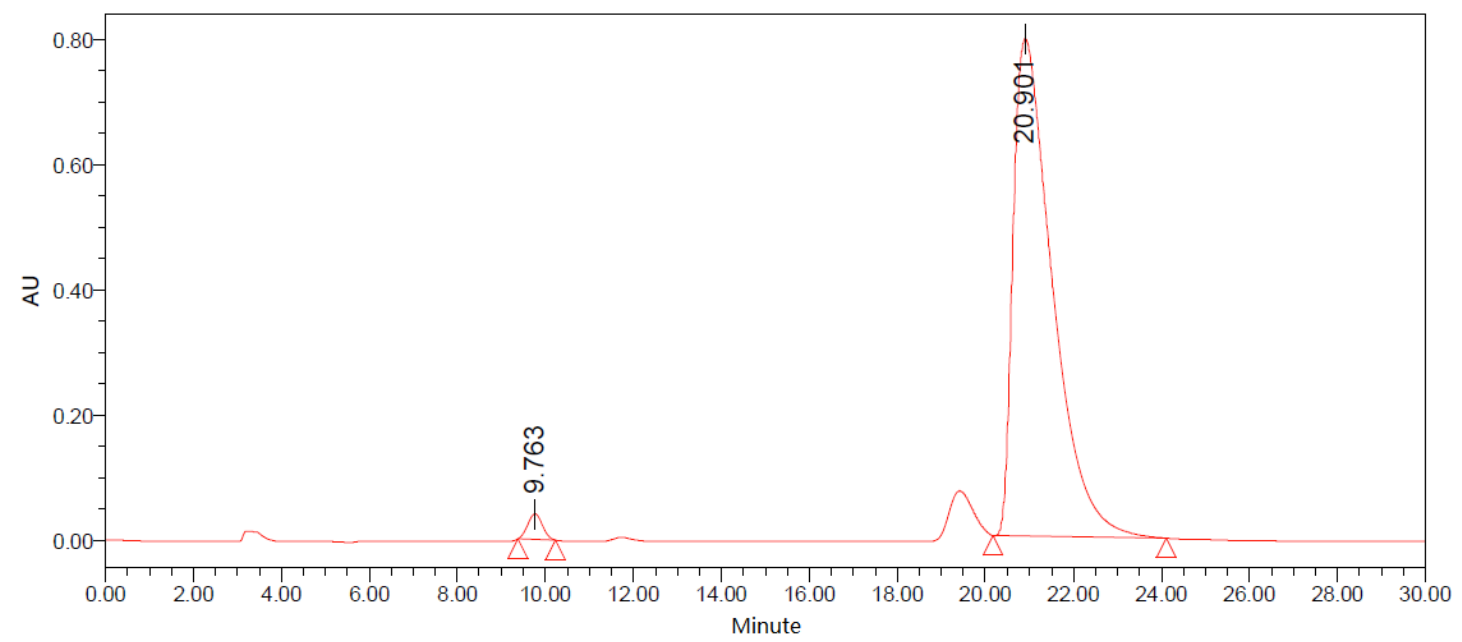

\begin{tabular}{|c|c|c|c|c|c|c|c|}
\hline & $\begin{array}{l}\text { RT } \\
\text { (Min) }\end{array}$ & $\begin{array}{c}\text { Area } \\
\left(\mu U^{*} s\right)\end{array}$ & $\%$ Area & $\begin{array}{l}\text { He ight } \\
(\mu \mathrm{U})\end{array}$ & $\%$ Height & $\begin{array}{l}\text { Start Time } \\
\text { (Min) }\end{array}$ & $\begin{array}{l}\text { End Time } \\
\text { (Min) }\end{array}$ \\
\hline 1 & 9.763 & 948900 & 1.90 & 40973 & 4.91 & 9.372 & 10.222 \\
\hline 2 & 20.901 & 49032049 & 98.10 & 794062 & 95.09 & 20.173 & 24.105 \\
\hline
\end{tabular}



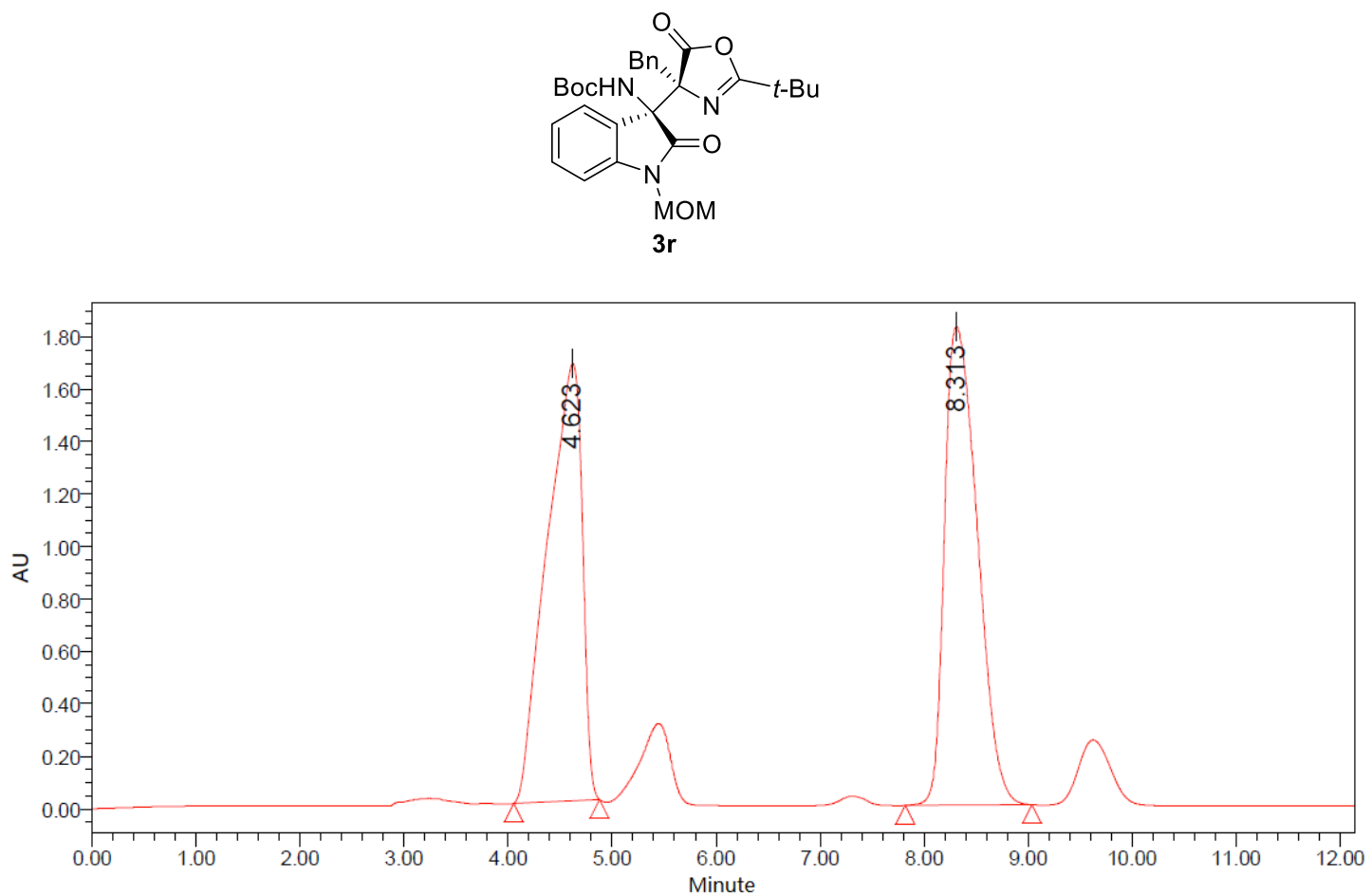

\begin{tabular}{|l|c|c|r|r|r|r|r|}
\hline & $\begin{array}{c}\text { RT } \\
(\text { Min })\end{array}$ & $\begin{array}{c}\text { Area } \\
\left(\mu \mathrm{U}^{*} \mathrm{~s}\right)\end{array}$ & $\%$ Area & $\begin{array}{c}\text { He ight } \\
(\mu \mathrm{U})\end{array}$ & $\%$ He ight & $\begin{array}{c}\text { Start Time } \\
(\text { Min) }\end{array}$ & $\begin{array}{c}\text { End Time } \\
(\mathrm{Min})\end{array}$ \\
\hline 1 & 4.623 & 39105949 & 49.45 & 1666914 & 47.73 & 4.052 & 4.882 \\
\hline 2 & 8.313 & 39983796 & 50.55 & 1825706 & 52.27 & 7.822 & 9.040 \\
\hline
\end{tabular}

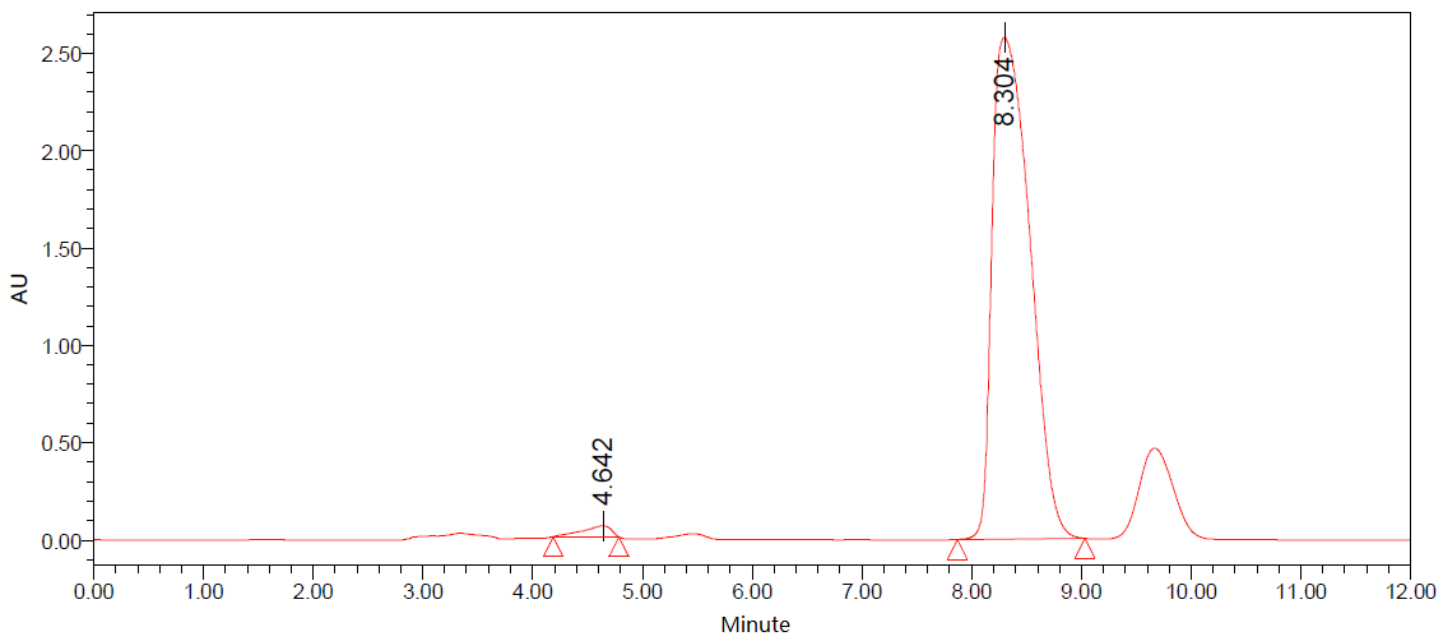

\begin{tabular}{|l|c|r|r|r|r|r|r|}
\hline & $\begin{array}{c}\mathrm{RT} \\
(\mathrm{Min})\end{array}$ & \multicolumn{1}{|c|}{$\begin{array}{c}\text { Area } \\
(\mu \mathrm{U} * \mathrm{~s})\end{array}$} & $\%$ Area & $\begin{array}{c}\text { He ight } \\
(\mu \mathrm{U})\end{array}$ & $\%$ He ight & $\begin{array}{c}\text { Start Time } \\
(\text { Min) }\end{array}$ & $\begin{array}{c}\text { End Time } \\
(\text { Min) }\end{array}$ \\
\hline 1 & 4.642 & 1029546 & 1.65 & 55409 & 2.10 & 4.187 & 4.783 \\
\hline 2 & 8.304 & 61494474 & 98.35 & 2578835 & 97.90 & 7.870 & 9.032 \\
\hline
\end{tabular}



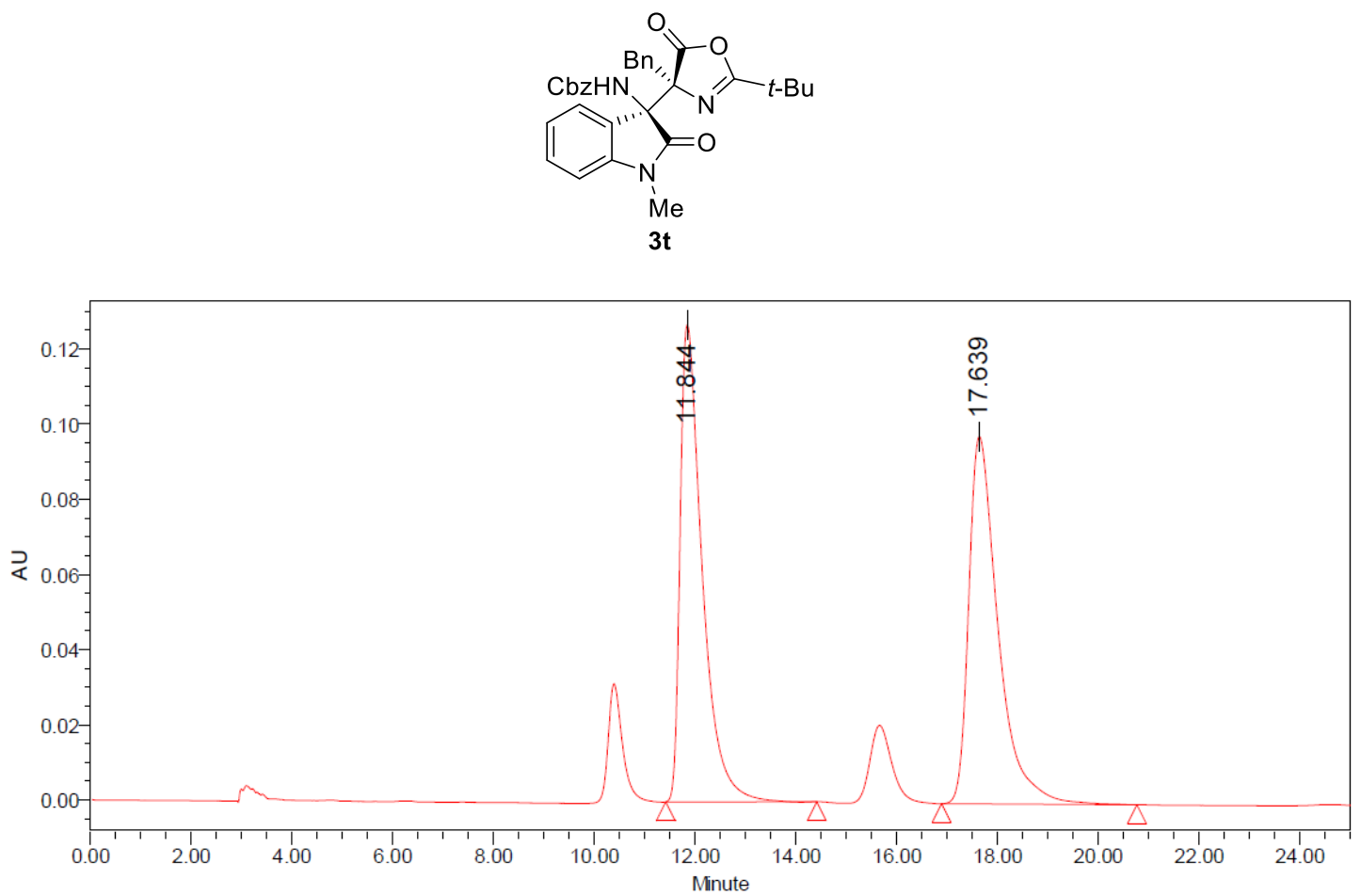

\begin{tabular}{|l|c|c|r|r|r|r|r|}
\hline & $\begin{array}{c}\text { RT } \\
(\text { Min) }\end{array}$ & $\begin{array}{c}\text { Area } \\
\left(\mu \mathrm{U}^{*} \mathrm{~s}\right)\end{array}$ & $\%$ Area & $\begin{array}{c}\text { He ight } \\
(\mu \mathrm{U})\end{array}$ & $\%$ He ight & $\begin{array}{c}\text { Start Time } \\
(\text { Min) }\end{array}$ & $\begin{array}{c}\text { End Time } \\
(\mathrm{Min})\end{array}$ \\
\hline 1 & 11.844 & 3859591 & 49.42 & 126970 & 56.51 & 11.417 & 14.412 \\
\hline 2 & 17.639 & 3949725 & 50.58 & 97725 & 43.49 & 16.888 & 20.765 \\
\hline
\end{tabular}

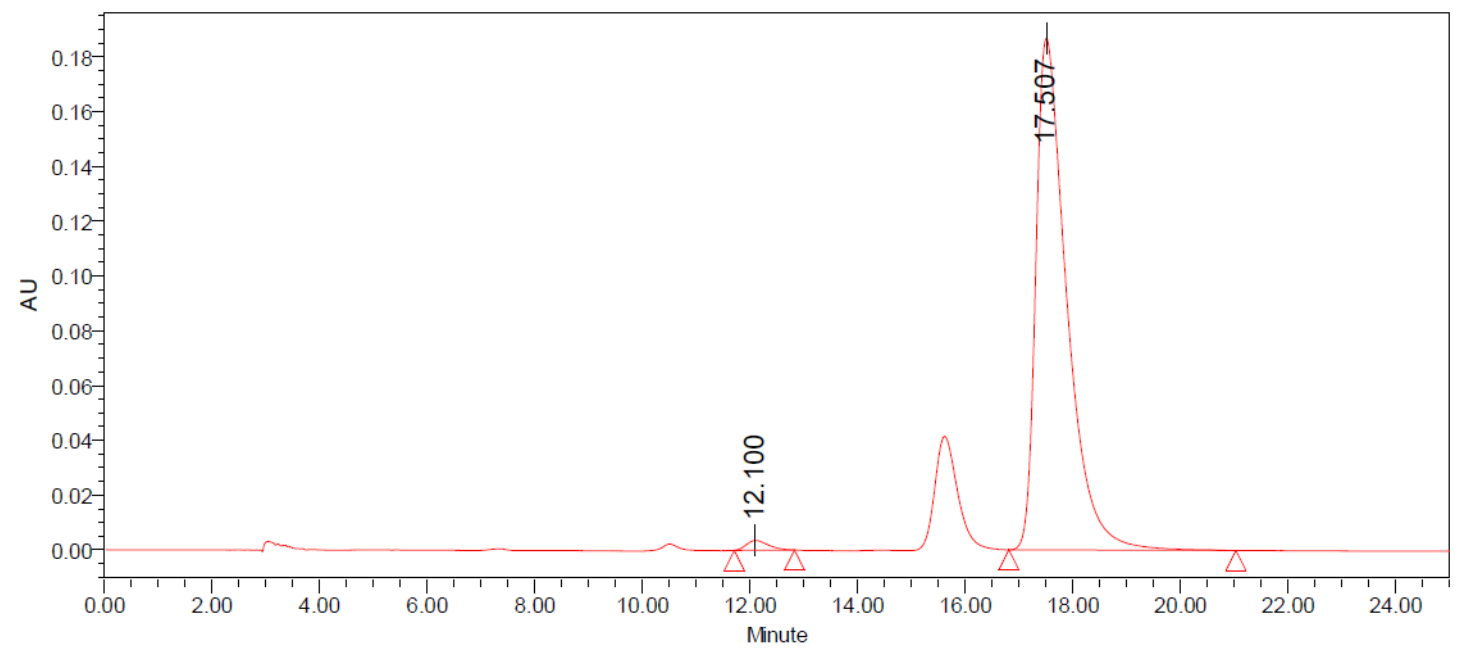

\begin{tabular}{|l|c|r|r|r|r|r|r|}
\hline & $\begin{array}{c}\text { RT } \\
(\text { Min) }\end{array}$ & \multicolumn{1}{|c|}{$\begin{array}{c}\text { Area } \\
\left(\mu U^{*}\right)\end{array}$} & $\%$ Area & $\begin{array}{c}\text { He ight } \\
(\mu \mathrm{U})\end{array}$ & $\%$ Height & $\begin{array}{c}\text { Start Time } \\
(\text { Min) }\end{array}$ & $\begin{array}{c}\text { End Time } \\
(\text { Min) }\end{array}$ \\
\hline 1 & 12.100 & 99822 & 1.32 & 3551 & 1.86 & 11.705 & 12.827 \\
\hline 2 & 17.507 & 7444090 & 98.68 & 186863 & 98.14 & 16.810 & 21.032 \\
\hline
\end{tabular}



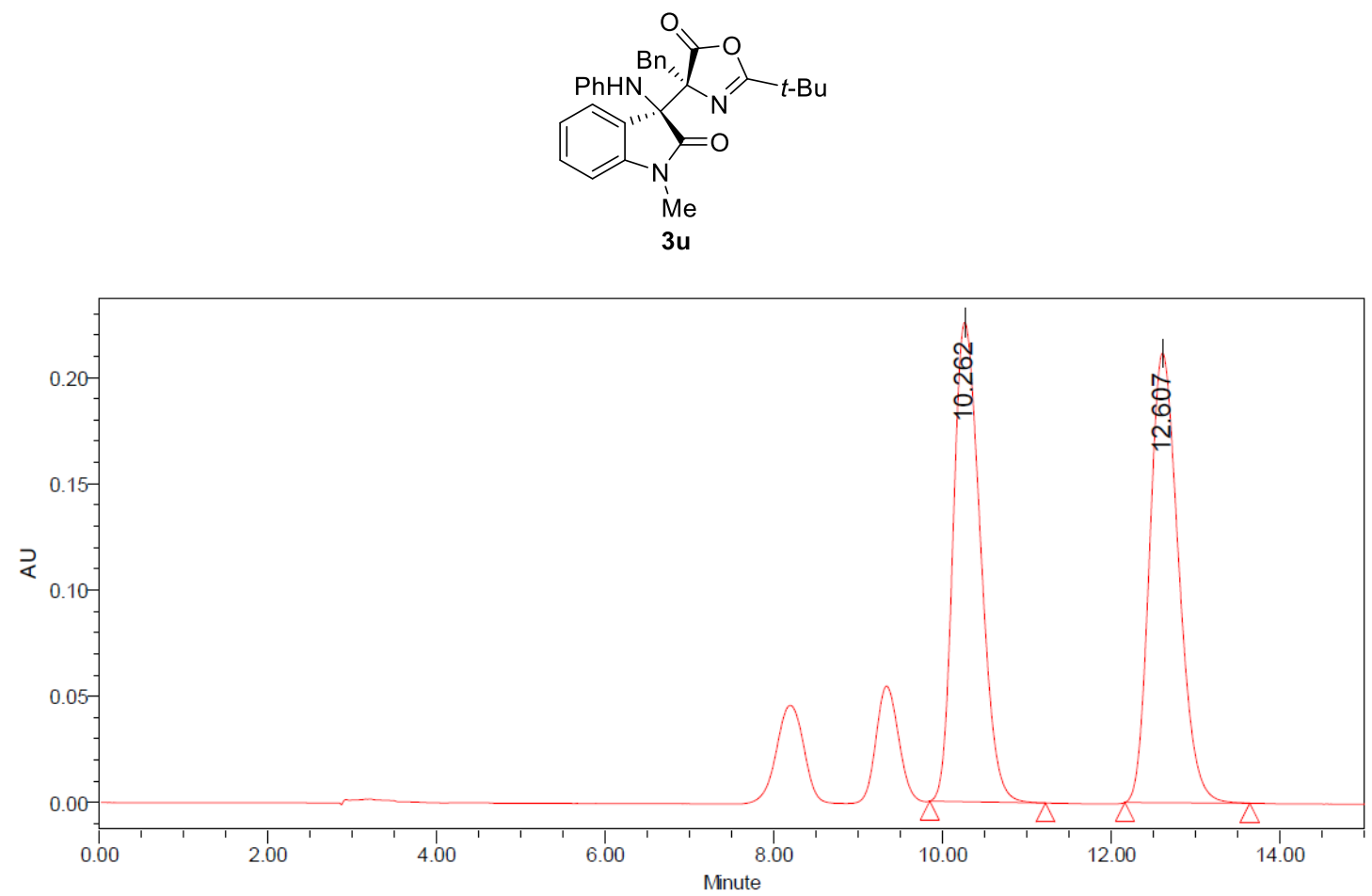

\begin{tabular}{|l|c|c|r|r|r|r|r|}
\hline & $\begin{array}{c}\mathbf{R} T \\
(\text { Min) }\end{array}$ & $\begin{array}{c}\text { Area } \\
\left(\mu \mathrm{U}^{*} \mathrm{~s}\right)\end{array}$ & $\%$ Area & $\begin{array}{c}\text { He ight } \\
(\mu \mathrm{U})\end{array}$ & $\%$ Height & $\begin{array}{c}\text { Start Time } \\
(\text { Min) }\end{array}$ & $\begin{array}{c}\text { End Time } \\
(\text { Min) }\end{array}$ \\
\hline 1 & 10.262 & 5025547 & 49.92 & 225513 & 51.61 & 9.847 & 11.218 \\
\hline 2 & 12.607 & 5042439 & 50.08 & 211450 & 48.39 & 12.162 & 13.642 \\
\hline
\end{tabular}

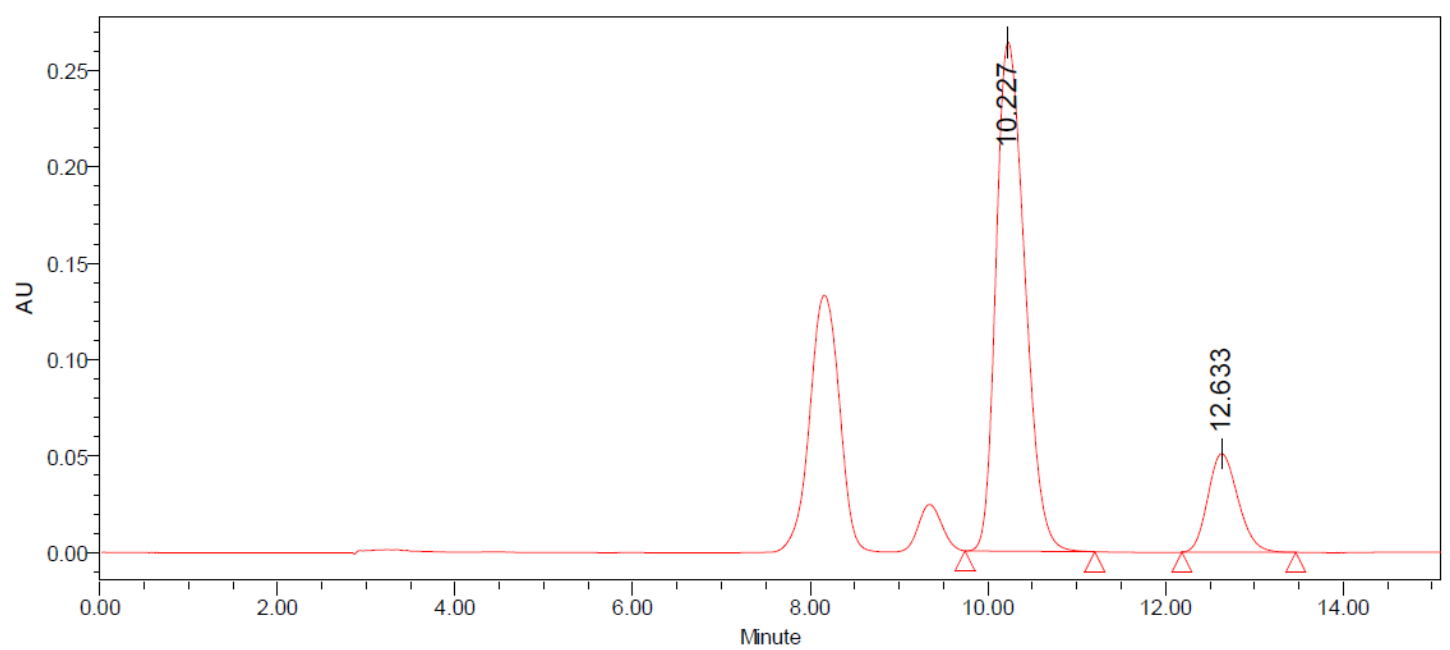

\begin{tabular}{|l|c|c|r|r|r|r|r|}
\hline & $\begin{array}{c}\text { RT } \\
(\text { Min) }\end{array}$ & $\begin{array}{c}\text { Area } \\
\left(\mu \mathrm{U}^{*} \mathrm{~s}\right)\end{array}$ & $\%$ Area & $\begin{array}{c}\text { He ight } \\
(\mu \mathrm{U})\end{array}$ & \% Height & $\begin{array}{c}\text { Start Time } \\
(\text { Min) }\end{array}$ & $\begin{array}{c}\text { End Time } \\
(\text { Min) }\end{array}$ \\
\hline 1 & 10.227 & 5948346 & 83.10 & 263944 & 83.77 & 9.742 & 11.203 \\
\hline 2 & 12.633 & 1210083 & 16.90 & 51149 & 16.23 & 12.185 & 13.467 \\
\hline
\end{tabular}



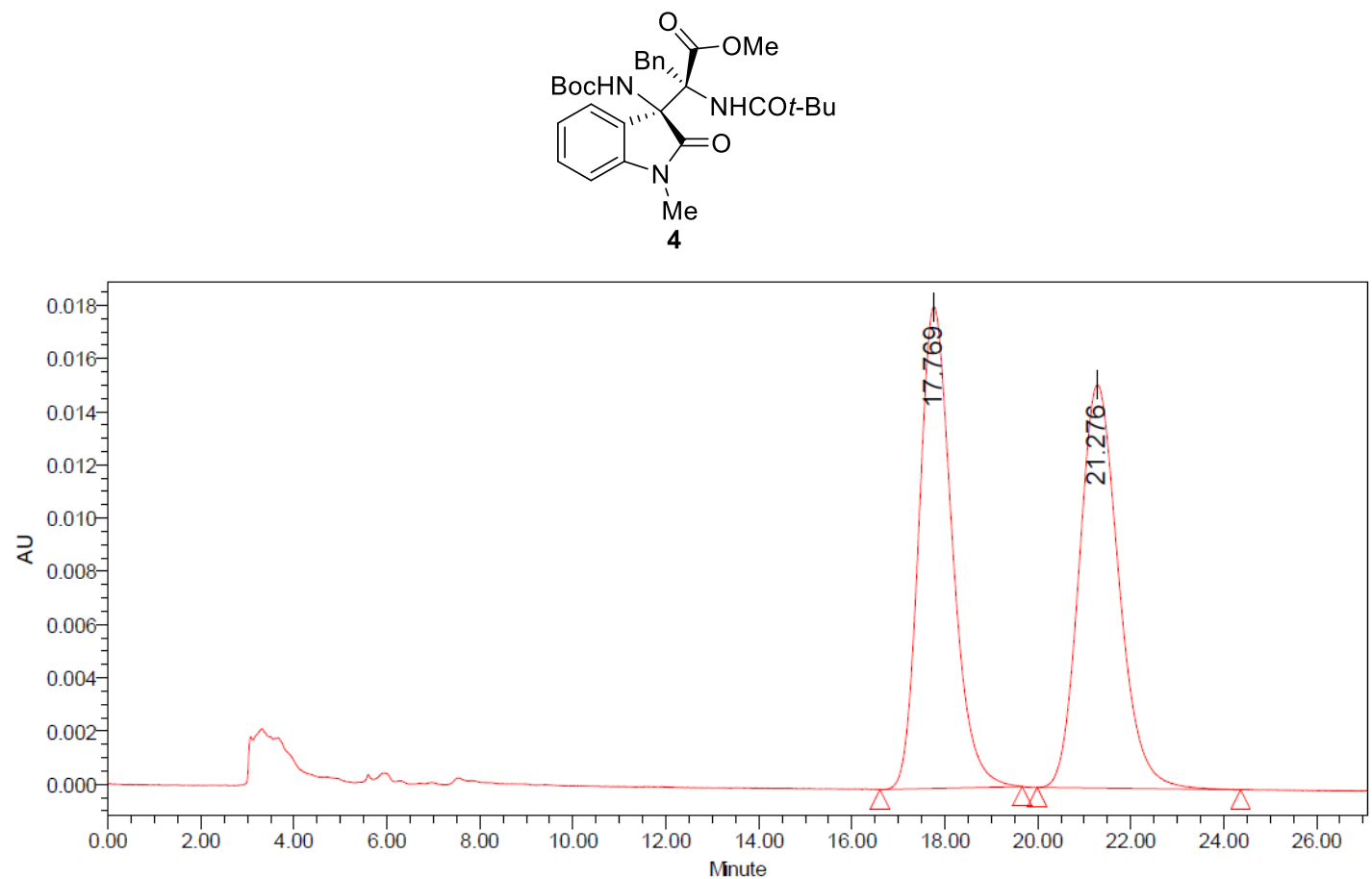

\begin{tabular}{|l|c|r|r|r|r|r|r|}
\hline & $\begin{array}{c}\text { RT } \\
(\text { Min) }\end{array}$ & $\begin{array}{c}\text { Area } \\
\left(\mu \mathrm{U}^{*} \mathrm{~s}\right)\end{array}$ & $\%$ Area & $\begin{array}{c}\text { He ight } \\
(\mu \mathrm{U})\end{array}$ & \% Height & $\begin{array}{c}\text { Start Time } \\
(\text { Min) }\end{array}$ & $\begin{array}{c}\text { End Time } \\
(\text { Min) }\end{array}$ \\
\hline 1 & 17.769 & 893209 & 49.95 & 18098 & 54.43 & 16.605 & 19.663 \\
\hline 2 & 21.276 & 894905 & 50.05 & 15152 & 45.57 & 19.987 & 24.365 \\
\hline
\end{tabular}

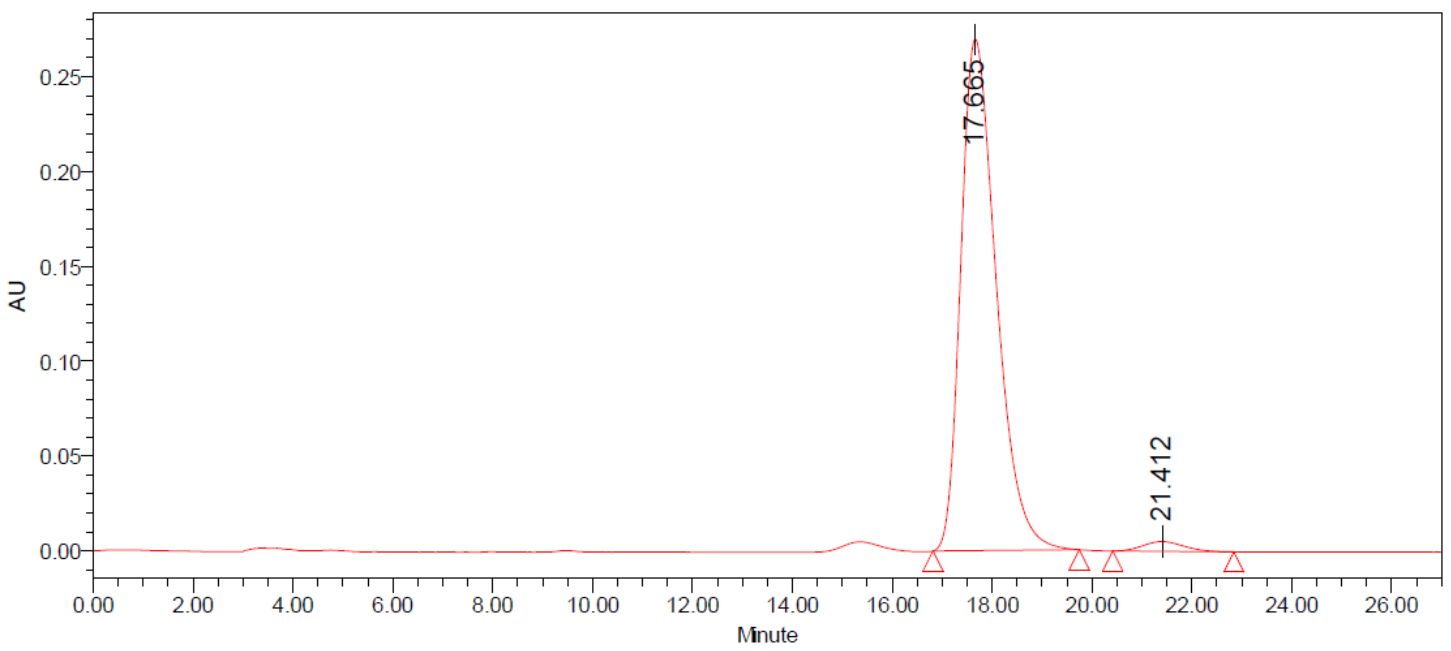

\begin{tabular}{|l|c|r|r|r|r|r|r|}
\hline & $\begin{array}{c}\text { RT } \\
(\mathrm{Min})\end{array}$ & \multicolumn{1}{|c|}{$\begin{array}{c}\text { Area } \\
\left(\mu \mathrm{U}{ }^{*} \mathrm{~s}\right)\end{array}$} & $\%$ Area & $\begin{array}{r}\text { He ight } \\
(\mu \mathrm{U})\end{array}$ & \% Height & $\begin{array}{c}\text { Start Time } \\
(\mathrm{Min})\end{array}$ & $\begin{array}{c}\text { End Time } \\
(\mathrm{Min})\end{array}$ \\
\hline 1 & 17.665 & 13305209 & 97.86 & 269622 & 98.13 & 16.817 & 19.748 \\
\hline 2 & 21.412 & 291341 & 2.14 & 5128 & 1.87 & 20.420 & 22.842 \\
\hline
\end{tabular}

